\title{
Dimension Theory of Iterated Function Systems
}

\author{
DE-JUN FENG \\ The Chinese University of Hong Kong \\ HUYI HU \\ Michigan State University
}

\begin{abstract}
Let $\left\{S_{i}\right\}_{i=1}^{\ell}$ be an iterated function system (IFS) on $\mathbb{R}^{d}$ with attractor $K$. Let $(\Sigma, \sigma)$ denote the one-sided full shift over the alphabet $\{1, \ldots, \ell\}$. We define the projection entropy function $h_{\pi}$ on the space of invariant measures on $\Sigma$ associated with the coding map $\pi: \Sigma \rightarrow K$ and develop some basic ergodic properties about it. This concept turns out to be crucial in the study of dimensional properties of invariant measures on $K$. We show that for any conformal IFS (respectively, the direct product of finitely many conformal IFSs), without any separation condition, the projection of an ergodic measure under $\pi$ is always exactly dimensional and its Hausdorff dimension can be represented as the ratio of its projection entropy to its Lyapunov exponent (respectively, the linear combination of projection entropies associated with several coding maps). Furthermore, for any conformal IFS and certain affine IFSs, we prove a variational principle between the Hausdorff dimension of the attractors and that of projections of ergodic measures. (c) 2008 Wiley Periodicals, Inc.
\end{abstract}

\section{Contents}

1. Introduction

2. Statement of the Main Results 4

3. Density Results about Conditional Measures 11

4. Projection Measure-Theoretic Entropies Associated with IFSs 18

5. Some Geometric Properties of $C^{1}$ IFSs 37

6. Estimates for Local Dimensions of Invariant Measures for $C^{1}$ IFSs 42

7. Proofs of Theorem 2.11 and Theorem $2.12 \quad 48$

8. A Variational Principle about Dimensions of Self-Conformal Sets 53

9. Proof of Theorem 2.15 56

10. A Final Remark about Infinite Noncontractive IFSs 62

Bibliography $\quad 63$

Communications on Pure and Applied Mathematics, 0001-0066 (PREPRINT)

(C) 2008 Wiley Periodicals, Inc. 


\section{Introduction}

Let $\left\{S_{i}: X \rightarrow X\right\}_{i=1}^{\ell}$ be a family of contractive maps on a nonempty closed set $X \subset \mathbb{R}^{d}$. Following Barnsley [2], we say that $\Phi=\left\{S_{i}\right\}_{i=1}^{\ell}$ is an iterated function system (IFS) on $X$. Hutchinson [27] showed that there is a unique nonempty compact set $K \subset X$, called the attractor of $\left\{S_{i}\right\}_{i=1}^{\ell}$, such that $K=\bigcup_{i=1}^{\ell} S_{i}(K)$. A probability measure $\mu$ on $\mathbb{R}^{d}$ is said to be exactly dimensional if there is a constant $C$ such that the local dimension

$$
d(\mu, x)=\lim _{r \rightarrow 0} \frac{\log \mu(B(x, r))}{\log r}
$$

exists and equals $C$ for $\mu$-a.e. $x \in \mathbb{R}^{d}$, where $B(x, r)$ denotes the closed ball of radius $r$ centered at $x$. It was shown by Young [65] that in such a case the Hausdorff dimension of $\mu$ is equal to $C$ (see also [14, 43, 51]).

The motivation of the paper is to study the Hausdorff dimension of an invariant measure $\mu$ (see Section 2 for precise meaning) for conformal and affine IFSs with overlaps. To deal with overlaps, we regard such a system as the image of a natural projection $\pi$ from the one-sided full shift space over $\ell$ symbols. Hence we obtain a dynamical system. We introduce a notion projection entropy, which plays a similar role as the classical entropy for IFSs satisfying the open set condition, and it becomes the classical entropy if the projection is finite to one. The concept of projection entropy turns out to be crucial in the study of dimensional properties of invariant measures on attractors of either conformal IFSs with overlaps or affine IFSs.

We develop some basic properties about projection entropy (Theorems 2.2 and 2.3). We prove that for conformal IFSs with overlaps, every ergodic measure $\mu$ is exactly dimensional and $d(\mu, x)$ is equal to the projection entropy divided by the Lyapunov exponent (Theorem 2.8). Furthermore, if $\Phi$ is a direct product of conformal IFSs (see Definition 2.10 for the precise meaning), then for every ergodic measure on $K$ the local dimension can be expressed by a Ledrappier-Young type formula in terms of projection entropies and Lyapunov exponents (Theorem 2.11). We also prove variational results about the Hausdorff dimension for conformal IFSs and certain affine IFSs (Theorems 2.13 and 2.15), which says that the Hausdorff dimension of the attractor $K$ is equal to the supremum of Hausdorff dimension of $\mu$ taking over all ergodic measures. The results we obtain cover some interesting cases such as $S_{i}(x)=\operatorname{diag}\left(\rho_{1}, \ldots, \rho_{d}\right) x+a_{i}$, where $i=1, \ldots, \ell$ and $\rho_{i}^{-1}$ are Pisot or Salem numbers and $a_{i} \in \mathbb{Z}^{d}$.

The problems of whether a given measure is exactly dimensional and whether the Hausdorff dimension of an attractor can be assumed or approximated by that of an invariant measure have been well studied in the literature for $C^{1+\alpha}$ conformal IFSs that satisfy the open set condition (cf. [6, 22, 49]). It is well-known that in such a case, any ergodic measure $\mu$ is exactly dimensional with the Hausdorff 
dimension given by the classic entropy divided by the Lyapunov exponent. Furthermore, there is a unique invariant measure $\mu$ with $\operatorname{dim}_{H}(\mu)=\operatorname{dim}_{H}(K)$, the Hausdorff dimension of $K$. However, the problems become much complicated and intractable without the assumption of the open set condition. Partial results have been obtained only for conformal IFSs that satisfy the finite-type condition (see [45] for the definition). In that case, a Bernoulli measure is exactly dimensional and its Hausdorff dimension may be expressed as the upper Lyapunov exponent of certain random matrices (see, e.g., [16, 17, 35, 37, 39]), and furthermore the Hausdorff dimension of $K$ can be computed (see, e.g., [34, 45, 54]).

There are some results for certain special nonoverlapping affine IFSs. McMullen [44] and Bedford [5] independently computed the Hausdorff dimension and the box dimension of the attractor of the following planar affine IFS:

$$
S_{i}(x)=\left[\begin{array}{cc}
n^{-1} & 0 \\
0 & k^{-1}
\end{array}\right] x+\left[\begin{array}{c}
a_{i} / n \\
b_{i} / k,
\end{array}\right], \quad i=1, \ldots, \ell,
$$

where all $a_{i}$ and $b_{i}$ are integers, $0 \leq a_{i}<n$, and $0 \leq b_{i}<k$. Furthermore, they showed that there is a Bernoulli measure of full Hausdorff dimension. This result was extended by Kenyon and Peres [33] to higher-dimensional self-affine Sierpinski sponges, for which ergodic measures are proved to be exactly dimensional with Hausdorff dimension given by a Ledrappier-Young type formula. Another extension of McMullen and Bedford's result to a broader class of planar affine IFSs $\left\{S_{i}\right\}_{i=1}^{\ell}$ was given by Gatzouras and Lalley [36], in which $S_{i}$ map the unit square $(0,1)^{2}$ into disjoint rectangles with sides parallel to the axes (where the longer sides are parallel to the $x$-axis; furthermore, once projected onto the $x$-axis, these rectangles are either identical or disjoint). Further extensions were given recently by Barański [1], Feng and Wang [19], Luzia [41], and Olivier [46]. For other related results, see, $[3,17,20,24,26,30,32,38,52,60]$.

Along another direction, in [11] Falconer gave a variational formula for the Hausdorff and box dimensions for "almost all" self-affine sets under some assumptions. This formula remains true under some weaker conditions [28, 61]. Käenmäki [29] proved that for "almost all" self-affine sets there exists an ergodic measure $m$ so that $m \circ \pi^{-1}$ is of full Hausdorff dimension.

Our arguments use ergodic theory and Rohlin's theory about conditional measures. The proofs of Theorem 2.6 and Theorem 2.11 are based on some ideas from the work of Ledrappier and Young [40] and techniques in analyzing the densities of conditional measures associated with overlapping IFSs.

So far we have restricted ourselves on the study of finite contractive IFSs. However, we point out that part of our results remain valid for certain noncontractive infinite IFSs (see Section 10 for details).

The paper is organized as follows: The main results are given in Section 2. In Section 3, we prove some density results about conditional measures. In Section 4, we investigate the properties of projection entropy and prove Theorems 2.2 and 2.3. In Section 5, we give some local geometric properties of a $C^{1}$ IFS. In Section 6, we 
prove a generalized version of Theorem 2.6, which is based on a key proposition (Proposition 6.1) about the densities of conditional measures. In Section 7, we prove Theorems 2.11 and 2.12. In Section 8, we prove Theorem 2.13, and in Section 9 we prove Theorem 2.15. In Section 10 we give a remark regarding certain noncontractive infinite IFSs.

\section{Statement of the Main Results}

Let $\left\{S_{i}\right\}_{i=1}^{\ell}$ be an IFS on a closed set $X \subset \mathbb{R}^{d}$. Denote by $K$ its attractor. Let $\Sigma=\{1, \ldots, \ell\}^{\mathbb{N}}$ be associated with the left shift $\sigma$ (cf. [9]). Let $\mathcal{M}_{\sigma}(\Sigma)$ denote the space of $\sigma$-invariant measures on $\Sigma$ endowed with the weak-star topology. Let $\pi: \Sigma \rightarrow K$ be the canonical projection defined by

$$
\{\pi(x)\}=\bigcap_{n=1}^{\infty} S_{x_{1}} \circ \cdots \circ S_{x_{n}}(K) \quad \text { where } x=\left(x_{i}\right)_{i=1}^{\infty} .
$$

A measure $\mu$ on $K$ is called invariant (respectively, ergodic) for the IFS if there is an invariant (respectively, ergodic) measure $v$ on $\Sigma$ such that $\mu=v \circ \pi^{-1}$.

Let $(\Omega, \mathcal{F}, \nu)$ be a probability space. For a sub- $\sigma$-algebra $\mathcal{A}$ of $\mathcal{F}$ and $f \in$ $L^{1}(\Omega, \mathcal{F}, \nu)$, we denote by $\mathbf{E}_{v}(f \mid \mathcal{A})$ the conditional expectation of $f$ given $\mathcal{A}$. For a countable $\mathcal{F}$-measurable partition $\xi$ of $\Omega$, we denote by $\mathbf{I}_{v}(\xi \mid \mathcal{A})$ the conditional information of $\xi$ given $\mathcal{A}$, which is given by the formula

$$
\mathbf{I}_{v}(\xi \mid \mathcal{A})=-\sum_{A \in \xi} \chi_{A} \log \mathbf{E}_{v}\left(\chi_{A} \mid \mathcal{A}\right)
$$

where $\chi_{A}$ denotes the characteristic function on $A$. The conditional entropy of $\xi$ given $\mathcal{A}$, written $H_{\nu}(\xi \mid \mathcal{A})$, is defined by the formula

$$
H_{\nu}(\xi \mid \mathcal{A})=\int \mathbf{I}_{\nu}(\xi \mid \mathcal{A}) d \nu
$$

(See [48] for more details.) The above information and entropy are unconditional when $\mathcal{A}=\mathcal{N}$, the trivial $\sigma$-algebra consisting of sets of measure zero and one, and in this case we write

$$
\mathbf{I}_{\nu}(\xi \mid \mathcal{N})=: \mathbf{I}_{\nu}(\xi) \quad \text { and } \quad H_{\nu}(\xi \mid \mathcal{N})=: H_{\nu}(\xi)
$$

Now we consider the space $(\Sigma, \mathcal{B}(\Sigma), m)$, where $\mathcal{B}(\Sigma)$ is the Borel $\sigma$-algebra on $\Sigma$ and $m \in \mathcal{M}_{\sigma}(\Sigma)$. Let $\mathcal{P}$ denote the Borel partition

$$
\mathcal{P}=\{[j]: 1 \leq j \leq \ell\}
$$

of $\Sigma$, where $[j]=\left\{\left(x_{i}\right)_{i=1}^{\infty} \in \Sigma: x_{1}=j\right\}$. Let $\mathcal{I}$ denote the $\sigma$-algebra

$$
\mathcal{I}=\left\{B \in \mathcal{B}(\Sigma): \sigma^{-1} B=B\right\} .
$$

For convenience, we use $\gamma$ to denote the Borel $\sigma$-algebra $\mathcal{B}\left(\mathbb{R}^{d}\right)$ on $\mathbb{R}^{d}$. 
DEFINITION 2.1 For any $m \in \mathcal{M}_{\sigma}(\Sigma)$, we call

$$
h_{\pi}(\sigma, m):=H_{m}\left(\mathcal{P} \mid \sigma^{-1} \pi^{-1} \gamma\right)-H_{m}\left(\mathcal{P} \mid \pi^{-1} \gamma\right)
$$

the projection entropy of $m$ under $\pi$ with respect to $\left\{S_{i}\right\}_{i=1}^{\ell}$, and we call

$$
h_{\pi}(\sigma, m, x):=\mathbf{E}_{m}(f \mid \mathcal{I})(x)
$$

the local projection entropy of $m$ at $x$ under $\pi$ with respect to $\left\{S_{i}\right\}_{i=1}^{\ell}$, where $f$ denotes the function $\mathbf{I}_{m}\left(\mathcal{P} \mid \sigma^{-1} \pi^{-1} \gamma\right)-\mathbf{I}_{m}\left(\mathcal{P} \mid \pi^{-1} \gamma\right)$.

It is clear that $h_{\pi}(\sigma, m)=\int h_{\pi}(\sigma, m, x) d m(x)$. Our first result is the following theorem:

THEOREM 2.2 Let $\left\{S_{i}\right\}_{i=1}^{\ell}$ be an IFS. Then

(i) For any $m \in \mathcal{M}_{\sigma}(\Sigma)$, we have $0 \leq h_{\pi}(\sigma, m) \leq h(\sigma, m)$, where $h(\sigma, m)$ denotes the classical measure-theoretic entropy of $m$ associated with $\sigma$.

(ii) The map $m \mapsto h_{\pi}(\sigma, m)$ is affine on $\mathcal{M}_{\sigma}(\Sigma)$. Furthermore, if $m=$ $\int v d \mathbb{P}(v)$ is the ergodic decomposition of $m$, we have

$$
h_{\pi}(\sigma, m)=\int h_{\pi}(\sigma, v) d \mathbb{P}(v)
$$

(iii) For any $m \in \mathcal{M}_{\sigma}(\Sigma)$, we have

$$
\lim _{n \rightarrow \infty} \frac{1}{n} \mathbf{I}_{m}\left(\mathcal{P}_{0}^{n-1} \mid \pi^{-1} \gamma\right)(x)=h(\sigma, m, x)-h_{\pi}(\sigma, m, x)
$$

for $m$-a.e. $x \in \Sigma$, where $h(\sigma, m, x)$ denotes the local entropy of $m$ at $x$; that is, $h(\sigma, m, x)=\mathbf{I}_{m}\left(\mathcal{P} \mid \sigma^{-1} \mathcal{B}(\Sigma)\right)(x)$.

Part (iii) of the theorem is an analogue of the classical relativized ShannonMcMillan-Breiman theorem (see, e.g., [8, lemma 4.1]). However, we should notice that the sub- $\sigma$-algebra $\pi^{-1} \gamma$ in our consideration is not $\sigma$-invariant in general (see Remark 4.11).

Part (iii) also implies that if the map $\pi: \Sigma \rightarrow K$ is finite to one, then

$$
h_{\pi}(\sigma, m)=h(\sigma, m)
$$

for any $m \in \mathcal{M}_{\sigma}(\Sigma)$. In Section 4, we will present a sufficient and necessary condition for the equality (see Corollary 4.16). However, for general overlapping IFSs, the projection entropy can be strictly less than the classical entropy.

In our next theorem, we give a geometric characterization of the projection entropy for certain affine IFSs, which will be used later in the proof of our variational results about the Hausdorff and box dimensions of self-affine sets.

THEOREM 2.3 Assume that $\Phi=\left\{S_{i}\right\}_{i=1}^{\ell}$ is an IFS on $\mathbb{R}^{d}$ of the form

$$
S_{i}(x)=A x+c_{i}, \quad i=1, \ldots, \ell,
$$

where $A$ is a $d \times d$ nonsingular contractive real matrix and $c_{i} \in \mathbb{R}^{d}$. Let $K$ denote the attractor of $\Phi$. Let $\mathcal{Q}$ denote the partition $\left\{[0,1)^{d}+\alpha: \alpha \in \mathbb{Z}^{d}\right\}$ of $\mathbb{R}^{d}$. For $n=0,1, \ldots$, we set $\mathcal{Q}_{n}=\left\{A^{n} Q: Q \in \mathcal{Q}\right\}$. Then 
(i) For any $m \in \mathcal{M}_{\sigma}(\Sigma)$, we have

$$
h_{\pi}(\sigma, m)=\lim _{n \rightarrow \infty} \frac{H_{m}\left(\pi^{-1} \mathcal{Q}_{n}\right)}{n} .
$$

(ii) Moreover,

$$
\lim _{n \rightarrow \infty} \frac{\log \#\left\{Q \in \mathcal{Q}: A^{n} Q \cap K \neq \varnothing\right\}}{n}=\sup \left\{h_{\pi}(\sigma, m): m \in \mathcal{M}_{\sigma}(\Sigma)\right\} .
$$

To give the applications of projection entropy in dimension theory of IFSs, we need some more notation and definitions.

Definition $2.4\left\{S_{i}: X \rightarrow X\right\}_{i=1}^{\ell}$ is called a $C^{1} I F S$ on a compact set $X \subset \mathbb{R}^{d}$ if each $S_{i}$ extends to a contracting $C^{1}$-diffeomorphism $S_{i}: U \rightarrow S_{i}(U) \subset U$ on an open set $U \supset X$.

For any $d \times d$ real matrix $M$, we use $\|M\|$ to denote the usual norm of $M$, and $\llbracket M \rrbracket$ the smallest singular value of $M$, i.e.,

$$
\begin{aligned}
\|M\| & =\max \left\{|M v|: v \in \mathbb{R}^{d},|v|=1\right\} \quad \text { and } \\
\llbracket M \rrbracket & =\min \left\{|M v|: v \in \mathbb{R}^{d},|v|=1\right\} .
\end{aligned}
$$

Definition 2.5 Let $\left\{S_{i}\right\}_{i=1}^{\ell}$ be a $C^{1}$ IFS. For $x=\left(x_{j}\right)_{j=1}^{\infty} \in \Sigma$, the upper and lower Lyapunov exponents of $\left\{S_{i}\right\}_{i=1}^{\ell}$ at $x$ are defined respectively by

$$
\begin{aligned}
& \bar{\lambda}(x)=-\liminf _{n \rightarrow \infty} \frac{1}{n} \log \| S_{x_{1} \cdots x_{n}}^{\prime}\left(\pi \sigma^{n} x\right) \rrbracket, \\
& \underline{\lambda}(x)=-\limsup _{n \rightarrow \infty} \frac{1}{n} \log \left\|S_{x_{1} \cdots x_{n}}^{\prime}\left(\pi \sigma^{n} x\right)\right\|,
\end{aligned}
$$

where $S_{x_{1} \ldots x_{n}}^{\prime}\left(\pi \sigma^{n} x\right)$ denotes the differential of $S_{x_{1} \cdots x_{n}}:=S_{x_{1}} \circ \cdots \circ S_{x_{n}}$ at $\pi \sigma^{n} x$. When $\bar{\lambda}(x)=\underline{\lambda}(x)$, the common value, denoted by $\lambda(x)$, is called the Lyapunov exponent of $\left\{S_{i}\right\}_{i=1}^{\ell}$ at $x$.

It is easy to check that both $\bar{\lambda}$ and $\underline{\lambda}$ are positive-valued $\sigma$-invariant functions on $\Sigma$ (i.e., $\bar{\lambda}=\bar{\lambda} \circ \sigma$ and $\underline{\lambda}=\underline{\lambda} \circ \sigma$ ). Recall that for a probability measure $\mu$ on $\mathbb{R}^{d}$, the local upper and lower dimensions are defined, respectively, by

$$
\bar{d}(\mu, x)=\limsup _{r \rightarrow 0} \frac{\log \mu(B(x, r))}{\log r}, \quad \underline{d}(\mu, x)=\liminf _{r \rightarrow 0} \frac{\log \mu(B(x, r))}{\log r},
$$

where $B(x, r)$ denotes the closed ball of radius $r$ centered at $x$. If $\bar{d}(\mu, x)=$ $\underline{d}(\mu, x)$, the common value is denoted as $d(\mu, x)$ and is called the local dimension of $m$ at $x$.

The following theorem gives an estimate of local dimensions of invariant measures on the attractor of an arbitrary $C^{1}$ IFS without any separation condition. 
THEOREM 2.6 Let $\left\{S_{i}\right\}_{i=1}^{\ell}$ be a $C^{1}$ IFS with attractor $K$. Then for $\mu=m \circ \pi^{-1}$, where $m \in \mathcal{M}_{\sigma}(\Sigma)$, we have the following estimates:

$$
\bar{d}(\mu, \pi x) \leq \frac{h_{\pi}(\sigma, m, x)}{\underline{\lambda}(x)} \quad \text { and } \quad \underline{d}(\mu, \pi x) \geq \frac{h_{\pi}(\sigma, m, x)}{\bar{\lambda}(x)} \quad \text { form-a.e. } x \in \Sigma \text {, }
$$

where $h_{\pi}(\sigma, m, x)$ denotes the local projection entropy of $m$ at $x$ under $\pi$ (see Definition 2.1). In particular, if $m$ is ergodic, we have

$$
\frac{h_{\pi}(\sigma, m)}{\int \bar{\lambda} d m} \leq \underline{d}(\mu, z) \leq \bar{d}(\mu, z) \leq \frac{h_{\pi}(\sigma, m)}{\int \underline{\lambda} d m} \quad \text { for } \mu \text {-a.e. } z \in K .
$$

Definition 2.7 Let $\left\{S_{i}\right\}_{i=1}^{\ell}$ be a $C^{1}$ IFS and $m \in \mathcal{M}_{\sigma}(\Sigma)$. We say that $\left\{S_{i}\right\}_{i=1}^{\ell}$ is $m$-conformal if $\lambda(x)$ exists (i.e., $\bar{\lambda}(x)=\underline{\lambda}(x))$ for $m$-a.e. $x \in \Sigma$.

As a direct application of Theorem 2.6, we have the following:

THEOREM 2.8 Assume that $\left\{S_{i}\right\}_{i=1}^{\ell}$ is $m$-conformal for some $m \in \mathcal{M}_{\sigma}(\Sigma)$. Let $\mu=m \circ \pi^{-1}$. Then we have

$$
d(\mu, \pi x)=\frac{h_{\pi}(\sigma, m, x)}{\lambda(x)} \quad \text { for m-a.e. } x \in \Sigma .
$$

In particular, if $m$ is ergodic, we have

$$
d(\mu, z)=\frac{h_{\pi}(\sigma, m)}{\int \lambda d m} \quad \text { for } \mu \text {-a.e. } z \in K .
$$

Recall that $S: U \rightarrow S(U)$ is a conformal map if $S^{\prime}(x): \mathbb{R}^{d} \rightarrow \mathbb{R}^{d}$ satisfies $\left\|S^{\prime}(x)\right\| \neq 0$ and $\left|S^{\prime}(x) y\right|=\left\|S^{\prime}(x)\right\||y|$ for all $x \in U$ and $y \in \mathbb{R}^{d}$.

DEFINITION 2.9 A $C^{1}$ IFS $\left\{S_{i}\right\}_{i=1}^{\ell}$ is said to be weakly conformal if

$$
\frac{1}{n}\left(\log \rrbracket S_{x_{1} \cdots x_{n}}^{\prime}\left(\pi \sigma^{n} x\right) \rrbracket-\log \left\|S_{x_{1} \cdots x_{n}}^{\prime}\left(\pi \sigma^{n} x\right)\right\|\right)
$$

converges to 0 uniformly on $\Sigma$ as $n$ tends to $\infty$. We say that $\left\{S_{i}\right\}_{i=1}^{\ell}$ is conformal if each $S_{i}$ extends to a conformal map $S_{i}: U \rightarrow S_{i}(U) \subset U$ on an open set $U \supset K$, where $K$ is the attractor of $\left\{S_{i}\right\}_{i=1}^{\ell}$.

By definition, a conformal IFS is always weakly conformal. Furthermore, a weakly conformal IFS is $m$-conformal for each $m \in \mathcal{M}_{\sigma}(\Sigma)$ (see Proposition 5.6(ii)). There are some natural examples of weakly conformal IFSs that are not conformal. For instance, let $S_{i}(x)=A_{i} x+a_{i}, i=1, \ldots, \ell$, such that, for each $i$, $A_{i}$ is a contracting linear map with eigenvalues equal to each other in modulus, and $A_{i} A_{j}=A_{j} A_{i}$ for different $i, j$. Then such an IFS is always weakly conformal but not necessarily conformal. The first conclusion follows from the asymptotic behavior

$$
\lim _{n \rightarrow \infty}\left\|A_{i}^{n} \rrbracket^{1 / n}=\lim _{n \rightarrow \infty}\right\| A_{i}^{n} \|^{1 / n}=\rho\left(A_{i}\right), \quad i=1, \ldots, \ell,
$$

where $\rho\left(A_{i}\right)$ denotes the spectral radius of $A_{i}$ (cf. [64]). 
Theorem 2.8 verifies the existence of local dimensions for invariant measures on the attractor of an arbitrary weakly conformal IFS without any separation assumption. We point out that the exact dimensionality for overlapping self-similar measures was first claimed by Ledrappier; nevertheless, no proof has been written out (cf. [50, p. 1619]). We remark that this property was also conjectured later by Fan, Lau, and Rao in [15].

We can extend the above result to a class of nonconformal IFSs.

Definition 2.10 Assume for $j=1, \ldots, k, \Phi_{j}:=\left\{S_{i, j}\right\}_{i=1}^{\ell}$ is a $C^{1}$ IFS defined on a compact set $X_{j} \subset \mathbb{R}^{q_{j}}$. Let $\Phi:=\left\{S_{i}\right\}_{i=1}^{\ell}$ be the IFS on $X_{1} \times \cdots \times X_{k} \subset$ $\mathbb{R}^{q_{1}} \times \cdots \times \mathbb{R}^{q_{k}}$ given by

$$
\begin{aligned}
& S_{i}\left(z_{1}, \ldots, z_{k}\right)=\left(S_{i, 1}\left(z_{1}\right), \ldots, S_{i, k}\left(z_{k}\right)\right), \\
& \quad i=1, \ldots, \ell, j=1, \ldots, k, z_{j} \in X_{j} .
\end{aligned}
$$

We say that $\Phi$ is the direct product of $\Phi_{1}, \ldots, \Phi_{k}$, and write $\Phi=\Phi_{1} \times \cdots \times \Phi_{k}$.

THEOREM 2.11 Let $\Phi=\left\{S_{i}\right\}_{i=1}^{\ell}$ be the direct product of $k C^{1} I F S s \Phi_{1}, \ldots, \Phi_{k}$. Let $\mu=m \circ \pi^{-1}$, where $m \in \mathcal{M}_{\sigma}(\Sigma)$. Assume that $\Phi_{1}, \ldots, \Phi_{k}$ are $m$-conformal. Then

(i) $d(\mu, z)$ exists for $\mu$-a.e. $z$.

(ii) Assume furthermore that $m$ is ergodic. Then $\mu$ is exactly dimensional. Let $\tau$ be a permutation on $\{1, \ldots, k\}$ such that

$$
\lambda_{\tau(1)} \leq \cdots \leq \lambda_{\tau(k)},
$$

where $\lambda_{j}=\int \lambda_{j}(x) d m(x)$, and $\lambda_{j}(x)$ denotes the Lyapunov exponent of $\Phi_{j}$ at $x \in \Sigma$. Then we have

$$
d(\mu, z)=\frac{h_{\pi_{1}}(\sigma, m)}{\lambda_{\tau(1)}}+\sum_{j=2}^{k} \frac{h_{\pi_{j}}(\sigma, m)-h_{\pi_{j-1}}(\sigma, m)}{\lambda_{\tau(j)}} \text { for } \mu \text {-a.e. } z,
$$

where $\pi_{j}$ denotes the canonical projection with respect to the IFS $\Phi_{\tau(1)} \times$ $\cdots \times \Phi_{\tau(j)}$, and $h_{\pi_{j}}(\sigma, m)$ denotes the projection entropy of $m$ under $\pi_{j}$.

We mention that fractals satisfying the conditions of the theorem include many interesting examples such as those studied in [5, 33, 36, 44].

As an application of Theorem 2.11, we have the following:

THEOREM 2.12 Let $\left\{S_{i}\right\}_{i=1}^{\ell}$ be an IFS on $\mathbb{R}^{d}$ of the form

$$
S_{i}(x)=A_{i} x+a_{i}, \quad i=1, \ldots, \ell,
$$

such that each $A_{i}$ is a nonsingular contracting linear map on $\mathbb{R}^{d}$, and $A_{i} A_{j}=$ $A_{j} A_{i}$ for any $1 \leq i, j \leq \ell$. Then for any ergodic measure $m$ on $\Sigma, \mu=m \circ \pi^{-1}$ is exactly dimensional. 
Indeed, under the assumption of Theorem 2.12, we can show that there is a nonsingular linear transformation $T$ on $\mathbb{R}^{d}$ such that the IFS $\left\{T \circ S_{i} \circ T^{-1}\right\}_{i=1}^{\ell}$ is the direct product of some weakly conformal IFSs. Hence we can apply Theorem 2.11 in this situation.

We remark that formula (2.7) provides an analogue of that for the Hausdorff dimension of $C^{1+\alpha}$ hyperbolic measures along the unstable (respectively, stable) manifold established by Ledrappier and Young [40].

The problem of the existence of local dimensions also has a long history in smooth dynamical systems. In [65], Young proved that an ergodic hyperbolic measure invariant under a $C^{1+\alpha}$ surface diffeomorphism is always exactly dimensional. For a measure $\mu$ in high-dimensional $C^{1+\alpha}$ systems, Ledrappier and Young [40] proved the existence of $\delta^{u}$ and $\delta^{s}$, the local dimensions along stable and unstable local manifolds, respectively, and the upper local dimension of $\mu$ is bounded by the sum of $\delta^{u}, \delta^{s}$, and the multiplicity of 0 as an exponent.

Eckmann and Ruelle [10] indicated that it is unknown whether the local dimension of $\mu$ is the sum of $\delta^{u}$ and $\delta^{s}$ if $\mu$ is a hyperbolic measure. Then the question was referred to as the Eckmann-Ruelle conjecture, and it was confirmed by Barreira, Pesin, and Schmeling in [4] 17 years later. Some partial dimensional results were obtained for measures invariant under hyperbolic endomorphism [58, 59]. Recently, Qian and Xie [53] proved the exact dimensionality of ergodic measures invariant under a $C^{2}$ expanding endomorphism on smooth Riemannian manifolds.

In the remaining part of this section, we present some variational results about the Hausdorff dimension and the box dimension of attractors of IFSs and that of invariant measures. First we consider conformal IFSs.

THEOREM 2.13 Let $K$ be the attractor of a weakly conformal IFS $\left\{S_{i}\right\}_{i=1}^{\ell}$. Then we have

$$
\begin{aligned}
\operatorname{dim}_{H} K & =\operatorname{dim}_{B} K \\
& =\sup \left\{\operatorname{dim}_{H} \mu: \mu=m \circ \pi^{-1}, m \in \mathcal{M}_{\sigma}(\Sigma), m \text { is ergodic }\right\} \\
& =\max \left\{\operatorname{dim}_{H} \mu: \mu=m \circ \pi^{-1}, m \in \mathcal{M}_{\sigma}(\Sigma)\right\} \\
& =\sup \left\{\frac{h_{\pi}(\sigma, m)}{\int \lambda d m}: m \in \mathcal{M}_{\sigma}(\Sigma)\right\},
\end{aligned}
$$

where $\operatorname{dim}_{B} K$ denotes the box dimension of $K$.

Equality (2.8) was first proved by Falconer [12] for $C^{1+\alpha}$ conformal IFS. It is not known whether the supremum in (2.9) and (2.10) can be attained in the general setting of Theorem 2.13. However, this is true if the IFS $\left\{S_{i}\right\}_{i=1}^{\ell}$ satisfies an additional separation condition defined as follows:

DEFINITION 2.14 An IFS $\left\{S_{i}\right\}_{i=1}^{\ell}$ on a compact set $X \subset \mathbb{R}^{d}$ is said to satisfy the asymptotically weak separation condition (AWSC), if

$$
\lim _{n \rightarrow \infty} \frac{1}{n} \log t_{n}=0
$$


where $t_{n}$ is given by

$$
t_{n}=\sup _{x \in \mathbb{R}^{d}} \#\left\{S_{u}: u \in\{1, \ldots, \ell\}^{n}, x \in S_{u}(K)\right\} ;
$$

here $K$ is the attractor of $\left\{S_{i}\right\}_{i=1}^{\ell}$.

The above definition was first introduced in [18] under a slightly different setting. For example, if $1 / \rho$ is a Pisot or Salem number, then the IFS $\left\{\rho x+a_{i}\right\}_{i=1}^{\ell}$ on $\mathbb{R}$, with $a_{i} \in \mathbb{Z}$, satisfies the AWSC (see proposition 5.3 and remark 5.5 in [18]). Recall that a real number $\beta>1$ is said to be a Salem number if it is an algebraic integer whose algebraic conjugates all have modulus not greater than 1 , with at least one being on the unit circle; $\beta>1$ is called a Pisot number if it is an algebraic integer whose algebraic conjugates all have modulus less than 1 . For instance, the largest root $(\approx 1.72208)$ of $x^{4}-x^{3}-x^{2}-x+1$ is a Salem number, and the golden ratio $(\sqrt{5}+1) / 2$ is a Pisot number. See [57] for more examples and properties of Pisot and Salem numbers. Under the AWSC assumption, we can show that the projection entropy map $m \mapsto h_{\pi}(\sigma, m)$ is upper semicontinuous on $\mathcal{M}_{\sigma}(\Sigma)$ (see Proposition 4.20) and, as a consequence, the supremum (2.9) and (2.10) can be attained at ergodic measures (see Remark 8.2).

Next we consider a class of affine IFSs.

THEOREM 2.15 Let $\Phi=\left\{S_{i}\right\}_{i=1}^{\ell}$ be an affine IFS on $\mathbb{R}^{d}$ given by

$$
S_{i}\left(x_{1}, \ldots, x_{d}\right)=\left(\rho_{1} x_{1}, \ldots, \rho_{d} x_{d}\right)+\left(a_{i, 1}, \ldots, a_{i, d}\right),
$$

where $\rho_{1}>\cdots>\rho_{d}>0$ and $a_{i, j} \in \mathbb{R}$. Let $K$ denote the attractor of $\Phi$, and write $\lambda_{j}=\log \left(1 / \rho_{j}\right)$ for $j=1, \ldots, d$ and $\lambda_{d+1}=\infty$. View $\Phi$ as the direct product of $\Phi_{1}, \ldots, \Phi_{d}$, where $\Phi_{j}=\left\{S_{i, j}\left(x_{j}\right)=\rho_{j} x_{j}+a_{i, j}\right\}_{i=1}^{\ell}$. Assume that $\Phi_{1} \times \cdots \times \Phi_{j}$ satisfies the AWSC for $j=1, \ldots, d$. Then we have

$$
\begin{aligned}
\operatorname{dim}_{H} K & =\max \left\{\operatorname{dim}_{H} \mu: \mu=m \circ \pi^{-1}, \text { m is ergodic }\right\} \\
& =\max \left\{\sum_{j=1}^{d}\left(\frac{1}{\lambda_{j}}-\frac{1}{\lambda_{j+1}}\right) h_{\pi_{j}}(\sigma, m): \text { m is ergodic }\right\},
\end{aligned}
$$

where $\pi_{j}$ is the canonical projection with respect to the IFS $\Phi_{1} \times \cdots \times \Phi_{j}$. Furthermore,

$$
\operatorname{dim}_{B} K=\sum_{j=1}^{d}\left(\frac{1}{\lambda_{j}}-\frac{1}{\lambda_{j+1}}\right) H_{j},
$$

where $H_{j}:=\max \left\{h_{\pi_{j}}(\sigma, m): m\right.$ is ergodic $\}$.

It is direct to check that if $\Phi_{j}$ satisfies the AWSC for each $1 \leq j \leq d$, then so does $\Phi_{1} \times \cdots \times \Phi_{j}$. Hence, for instance, the condition of Theorem 2.15 is fulfilled when $1 / \rho_{j}$ are Pisot numbers or Salem numbers and $\left(a_{i, 1}, \ldots, a_{i, d}\right) \in$ $\mathbb{Z}^{d}$. Different from the earlier works on the Hausdorff dimension of deterministic self-affine sets and self-affine measures (see, e.g., [1, 5, 26, 33, 36, 44, 46]), our 
model in Theorem 2.15 admits certain overlaps. The two variational results in Theorem 2.15 provide some new insights into the study of overlapping self-affine IFS. An interesting question is whether the results of Theorem 2.15 remain true without the AWSC assumption. It is related to the open problem of whether a nonconformal repeller carries an ergodic measure of full dimension (see [21] for a survey). We remark that, in the general case, we do have the following inequality (see Lemma 9.2):

$$
\underline{\operatorname{dim}}_{B} K \geq \sum_{j=1}^{d}\left(\frac{1}{\lambda_{j}}-\frac{1}{\lambda_{j+1}}\right) \sup \left\{h_{\pi_{j}}(\sigma, m): m \text { is ergodic }\right\} .
$$

Furthermore, Theorem 2.15 can be extended somewhat (see Remark 9.3 and Theorem 9.4).

\section{Density Results about Conditional Measures}

We prove some density results about conditional measures in this section. To begin with, we give a brief introduction to Rohlin's theory of Lebesgue spaces, measurable partitions, and conditional measures. The reader is referred to $[47,55]$ for more details.

A probability space $(X, \mathcal{B}, m)$ is called a Lebesgue space if it is isomorphic to a probability space that is the union of $[0, s], 0 \leq s \leq 1$, with Lebesgue measure and a countable number of atoms. Now let $(X, \mathcal{B}, \bar{m})$ be a Lebesgue space. A measurable partition $\eta$ of $X$ is a partition of $X$ such that, up to a set of measure zero, the quotient space $X / \eta$ is separated by a countable number of measurable sets $\left\{B_{i}\right\}$. The quotient space $X / \eta$ with its inherit probability space structure, written as $\left(X_{\eta}, \mathcal{B}_{\eta}, m_{\eta}\right)$, is again a Lebesgue space. Also, any measurable partition $\eta$ determines a sub- $\sigma$-algebra of $\mathcal{B}$, denoted by $\hat{\eta}$, whose elements are unions of elements of $\eta$. Conversely, any sub- $\sigma$-algebra $\mathcal{B}^{\prime}$ of $\mathcal{B}$ is also countably generated, say by $\left\{B_{i}^{\prime}\right\}$, and therefore all the sets of the form $\bigcap A_{i}$, where $A_{i}=B_{i}^{\prime}$ or its complement, form a measurable partition. In particular, $\mathcal{B}$ itself corresponds to a partition into single points. An important property of Lebesgue spaces and measurable partitions is the following:

THEOREM 3.1 (Rohlin [55]) Let $\eta$ be a measurable partition of a Lebesgue space $(X, \mathcal{B}, m)$. Then, for every $x$ in a set of full $m$-measure, there is a probability measure $m_{x}^{\eta}$ defined on $\eta(x)$, the element of $\eta$ containing $x$. These measures are uniquely characterized (up to sets of $m$-measure 0 ) by the following properties: if $A \subset X$ is a measurable set, then $x \mapsto m_{x}^{\eta}(A)$ is $\hat{\eta}$-measurable and $m(A)=$ $\int m_{x}^{\eta}(A) d m(x)$. These properties imply that for any $f \in L^{1}(X, \mathcal{B}, m), m_{x}^{\eta}(f)=$ $\mathbf{E}_{m}(f \mid \hat{\eta})(x)$ for $m$-a.e. $x$, and $m(f)=\int \mathbf{E}_{m}(f \mid \hat{\eta}) d m$.

The family of measures $\left\{m_{x}^{\eta}\right\}$ in the above theorem is called the canonical system of conditional measures associated with $\eta$. 
Throughout the remaining part of this section, we assume that $(X, \mathcal{B}, m)$ is a Lebesgue space. Let $\eta$ be a measurable partition of $X$, and let $\left\{m_{x}^{\eta}\right\}$ denote the corresponding canonical system of conditional measures. Suppose that $\pi: X \rightarrow$ $\mathbb{R}^{d}$ is a $\mathcal{B}$-measurable map. Denote $\gamma:=\mathcal{B}\left(\mathbb{R}^{d}\right)$, the Borel- $\sigma$-algebra on $\mathbb{R}^{d}$. For $y \in \mathbb{R}^{d}$, we use $B(y, r)$ to denote the closed ball in $\mathbb{R}^{d}$ of radius $r$ centered at $y$. Also, we denote for $x \in X$,

$$
B^{\pi}(x, r)=\pi^{-1} B(\pi x, r) .
$$

LeMma 3.2 Let $A \in \mathcal{B}$.

(i) The map $x \mapsto m_{x}^{\eta}\left(B^{\pi}(x, r) \cap A\right)$ is $\hat{\eta} \vee \pi^{-1} \gamma$-measurable for each $r>0$, where $\hat{\eta} \vee \pi^{-1} \gamma$ denotes the smallest sub- $\sigma$-algebra of $\mathcal{B}$ containing $\hat{\eta}$ and $\pi^{-1} \gamma$

(ii) The functions

$$
\liminf _{r \rightarrow 0} \frac{m_{x}^{\eta}\left(B^{\pi}(x, r) \cap A\right)}{m_{x}^{\eta}\left(B^{\pi}(x, r)\right)}, \quad \limsup _{r \rightarrow 0} \frac{m_{x}^{\eta}\left(B^{\pi}(x, r) \cap A\right)}{m_{x}^{\eta}\left(B^{\pi}(x, r)\right)},
$$

and

$$
\inf _{r>0} \frac{m_{x}^{\eta}\left(B^{\pi}(x, r) \cap A\right)}{m_{x}^{\eta}\left(B^{\pi}(x, r)\right)}
$$

are $\hat{\eta} \vee \pi^{-1} \gamma$-measurable, where we interpret $0 / 0=0$.

Proof: We first prove (i). Let $A \in \mathcal{B}$ and $r>0$. For $n \in \mathbb{N}$, let $\mathcal{D}_{n}$ denote the collection

$$
\mathcal{D}_{n}=\left\{\left[0,2^{-n}\right)^{d}+\alpha: \alpha \in 2^{-n} \mathbb{Z}^{d}\right\} .
$$

For $y \in \mathbb{R}^{d}$, denote

$$
W_{n}(y)=\bigcup_{Q \in \mathcal{D}_{n}: Q \cap B(y, r) \neq \varnothing} Q .
$$

Write $\mathcal{W}_{n}:=\left\{W_{n}(y): y \in \mathbb{R}^{d}\right\}$. It is clear that $\mathcal{W}_{n}$ is countable for each $n \in \mathbb{N}$. Furthermore, we have $W_{n}(y) \downarrow B(y, r)$ for each $y \in \mathbb{R}^{d}$ as $n \rightarrow \infty$, that is, $W_{n+1}(y) \subset W_{n}(y)$ and $\bigcap_{n=1}^{\infty} W_{n}(y)=B(y, r)$. As a consequence, we have $\pi^{-1} W_{n}(\pi x) \downarrow B^{\pi}(x, r)$ and hence

$$
m_{x}^{\eta}\left(B^{\pi}(x, r) \cap A\right)=\lim _{n \rightarrow \infty} m_{x}^{\eta}\left(\pi^{-1} W_{n}(\pi x) \cap A\right), \quad x \in X .
$$

Therefore to show that $x \mapsto m_{x}^{\eta}\left(B^{\pi}(x, r) \cap A\right)$ is $\hat{\eta} \vee \pi^{-1} \gamma$-measurable, it suffices to show that $x \mapsto m_{x}^{\eta}\left(\pi^{-1} W_{n}(\pi x) \cap A\right)$ is $\hat{\eta} \vee \pi^{-1} \gamma$-measurable for each $n \in \mathbb{N}$.

Fix $n \in \mathbb{N}$. For $F \in \mathcal{W}_{n}$, let $\Gamma_{n}(F)=\left\{x \in X: W_{n}(\pi x)=F\right\}$. Then $\Gamma_{n}(F) \in \pi^{-1} \gamma$. By Theorem 3.1, $m_{x}^{\eta}\left(\pi^{-1} F \cap A\right)$ is an $\hat{\eta}$-measurable function of $x$ for each $F \in \mathcal{W}_{n}$. However,

$$
m_{x}^{\eta}\left(\pi^{-1} W_{n}(\pi x) \cap A\right)=\sum_{F \in \mathcal{W}_{n}} \chi_{\Gamma_{n}(F)}(x) m_{x}^{\eta}\left(\pi^{-1} F \cap A\right) .
$$

Hence $m_{x}^{\eta}\left(\pi^{-1} W_{n}(\pi x) \cap A\right)$ is $\hat{\eta} \vee \pi^{-1} \gamma$-measurable; so is $m_{x}^{\eta}\left(B^{\pi}(x, r) \cap A\right)$. 
To see (ii), note that for $x \in \Sigma$ and $r>0$ satisfying $m_{x}^{\eta}\left(B^{\pi}(x, r)\right)>0$, we have

$$
\frac{m_{x}^{\eta}\left(B^{\pi}(x, r) \cap A\right)}{m_{x}^{\eta}\left(B^{\pi}(x, r)\right)}=\lim _{q \downarrow r: q \in \mathbb{Q}^{+}} \frac{m_{x}^{\eta}\left(B^{\pi}(x, q) \cap A\right)}{m_{x}^{\eta}\left(B^{\pi}(x, q)\right)} .
$$

Hence for the three limits in (ii), we can restrict $r$ to be positive rationals. It together with (i) yields the desired measurability.

Lemma 3.3 Let $A \in \mathcal{B}$. Then for $m$-a.e. $x \in X$,

$$
\lim _{r \rightarrow 0} \frac{m_{x}^{\eta}\left(B^{\pi}(x, r) \cap A\right)}{m_{x}^{\eta}\left(B^{\pi}(x, r)\right)}=\mathbf{E}_{m}\left(\chi_{A} \mid \hat{\eta} \vee \pi^{-1} \gamma\right)(x) .
$$

PROOF: Let $\bar{f}(x)$ and $\underline{f}(x)$ be the values obtained by taking the upper and lower limits in the left-hand side of (3.2). By Lemma 3.2, both $\bar{f}$ and $\underline{f}$ are $\hat{\eta} \vee \pi^{-1} \gamma$-measurable. In the following we only show that $\bar{f}(x)=\mathbf{E}_{m}\left(\chi_{A} \mid \hat{\eta} \vee\right.$ $\left.\pi^{-1} \gamma\right)(x)$ for $m$-a.e. $x$. The proof for $\underline{f}(x)=\mathbf{E}_{m}\left(\chi_{A} \mid \hat{\eta} \vee \pi^{-1} \gamma\right)(x)$ is similar.

We first prove that

$$
\int_{B \cap \pi^{-1} D} \bar{f} d m=\int_{B \cap \pi^{-1} D} \mathbf{E}_{m}\left(\chi_{A} \mid \hat{\eta} \vee \pi^{-1} \gamma\right) d m, \quad B \in \hat{\eta}, D \in \gamma .
$$

By Theorem 3.1, for any given $C \in \eta, m_{x}^{\eta}(x \in C)$ represents the same measure supported on $C$, which we rewrite as $m_{C}$. Fix $C \in \eta$. We define measures $\mu_{C}$ and $\nu_{C}$ on $\mathbb{R}^{d}$ by $\mu_{C}(E)=m_{C}\left(\pi^{-1} E \cap A\right)$ and $\nu_{C}(E)=m_{C}\left(\pi^{-1} E\right)$ for all $E \in \gamma$. It is clear that $\mu_{C} \ll v_{C}$. Define

$$
g_{C}(z)=\limsup _{r \rightarrow 0} \frac{\mu_{C}(B(z, r))}{v_{C}(B(z, r))}, \quad z \in \mathbb{R}^{d} .
$$

Then $\bar{f}(x)=g_{\eta(x)}(\pi x)$ for all $x \in \Sigma$. According to the differentiation theory of measures on $\mathbb{R}^{d}$ (see, e.g., [43, theorem 2.12]), $g_{C}=d \mu_{C} / d v_{C}, v_{C}$-a.e. Hence for each $D \in \gamma$, we have $\int_{D} g_{C}(z) d v_{C}(z)=\mu_{C}(D)$, i.e.,

$$
\int_{\pi^{-1} D} g_{C}(\pi y) d m_{C}(y)=\mu_{C}(D)=m_{C}\left(\pi^{-1} D \cap A\right) .
$$

That is,

$$
\int_{\pi^{-1} D} \bar{f} d m_{x}^{\eta}=m_{x}^{\eta}\left(\pi^{-1} D \cap A\right), \quad x \in X .
$$

To see (3.3), let $B \in \hat{\eta}$. Then

$$
\begin{aligned}
\int_{B \cap \pi^{-1} D} \bar{f} d m & =\int \chi_{B} \chi_{\pi^{-1} D} \bar{f} d m \\
& =\int \mathbf{E}_{m}\left(\chi_{B} \chi_{\pi^{-1} D} \bar{f} \mid \hat{\eta}\right) d m=
\end{aligned}
$$




$$
\begin{array}{ll}
=\int_{\chi_{B} \mathbf{E}_{m}\left(\chi_{\pi^{-1} D} \bar{f} \mid \hat{\eta}\right) d m} & \\
=\int_{B}\left(\int_{\pi^{-1} D} \bar{f} d m_{x}^{\eta}\right) d m(x) & \text { (by Theorem 3.1) } \\
=\int_{B} m_{x}^{\eta}\left(\pi^{-1} D \cap A\right) d m(x) & \text { (by (3.4)) } \\
=\int_{B} \chi_{B}(x) \mathbf{E}_{m}\left(\chi_{\pi^{-1} D \cap A} \mid \hat{\eta}\right)(x) d m(x) & \text { (by Theorem 3.1). }
\end{array}
$$

Thus we have

$$
\begin{aligned}
\int_{B \cap \pi^{-1} D} \bar{f} d m & =\int \mathbf{E}_{m}\left(\chi_{B} \chi_{\pi^{-1} D \cap A} \mid \hat{\eta}\right)(x) d m(x) \\
& =\int \chi_{B} \chi_{\pi^{-1} D \cap A} d m=m\left(B \cap \pi^{-1} D \cap A\right) \\
& =\int \mathbf{E}_{m}\left(\chi_{B \cap \pi^{-1} D} \chi_{A} \mid \hat{\eta} \vee \pi^{-1} \gamma\right) d m \\
& =\int \chi_{B \cap \pi^{-1} D} \mathbf{E}_{m}\left(\chi_{A} \mid \hat{\eta} \vee \pi^{-1} \gamma\right) d m \\
& =\int_{B \cap \pi^{-1} D} \mathbf{E}_{m}\left(\chi_{A} \mid \hat{\eta} \vee \pi^{-1} \gamma\right) d m
\end{aligned}
$$

This establishes (3.3).

Let $R=\bar{f}-\mathbf{E}_{m}\left(\chi_{A} \mid \hat{\eta} \vee \pi^{-1} \gamma\right)$. Then $R$ is $\hat{\eta} \vee \pi^{-1} \gamma$-measurable and

$$
\int_{B \cap \pi^{-1}(D)} R d m=0, \quad B \in \hat{\eta}, D \in \pi^{-1} \gamma .
$$

Denote $\mathcal{F}=\left\{B \cap \pi^{-1}(D): B \in \hat{\eta}, D \in \pi^{-1} \gamma\right\}$ and let

$$
\mathcal{F}^{\prime}=\left\{\bigcup_{i=1}^{k} F_{i}: k \in \mathbb{N}, F_{1}, \ldots, F_{k} \in \mathcal{F} \text { are disjoint }\right\} .
$$

It is clear that $\int_{F} R d m=0$ for all $F \in \mathcal{F}^{\prime}$. Moreover, it is a routine to check that $\mathcal{F}^{\prime}$ is an algebra that contains $\hat{\eta}$ and $\pi^{-1} \gamma$, and hence $\mathcal{F}^{\prime}$ generates the $\sigma$-algebra $\hat{\eta} \vee \pi^{-1} \gamma$.

We claim that $R=0 m$-a.e. Assume this is not true. Then there exists $\epsilon>0$ such that the set $\{R>\epsilon\}$ or $\{R<-\epsilon\}$ has positive $m$-measure. Without loss of generality, we assume that $m\{R>\epsilon\}>0$. Since $\mathcal{F}^{\prime}$ is an algebra that generates $\hat{\eta} \vee \pi^{-1} \gamma$, there exists a sequence $F_{i} \in \mathcal{F}^{\prime}$ such that $m\left(F_{i} \triangle\{R>\epsilon\}\right)$ tends to 0 as $i \rightarrow \infty$ (cf. [63, theorem 0.7]). We conclude that $\int_{F_{i}} R d m$ tends to $\int_{\{R>\epsilon\}} R d m>0$ as $i \rightarrow \infty$, which contradicts the fact that $\int_{F_{i}} R d m=0$. 
Remark 3.4.

(i) Letting $\eta=\mathcal{N}$ be the trivial partition of $X$ in the above lemma, we obtain

$$
\lim _{r \rightarrow 0} \frac{m\left(B^{\pi}(x, r) \cap A\right)}{m\left(B^{\pi}(x, r)\right)}=\mathbf{E}_{m}\left(\chi_{A} \mid \pi^{-1} \gamma\right)(x) \quad m \text {-a.e. }
$$

(ii) In general, $\mathbf{E}_{m_{x}^{\eta}}\left(\chi_{A} \mid \pi^{-1} \gamma\right)(x)=\mathbf{E}_{m}\left(\chi_{A} \mid \hat{\eta} \vee \pi^{-1} \gamma\right)(x) m$-a.e.; both of them equal

$$
\lim _{r \rightarrow 0} \frac{m_{x}^{\eta}\left(B^{\pi}(x, r) \cap A\right)}{m_{x}^{\eta}\left(B^{\pi}(x, r)\right)} \quad m \text {-a.e. }
$$

by (i).

Proposition 3.5 Let $\xi$ be a countable measurable partition of $X$. Then for $m$ a.e. $x \in X$,

$$
\lim _{r \rightarrow 0} \log \frac{m_{x}^{\eta}\left(B^{\pi}(x, r) \cap \xi(x)\right)}{m_{x}^{\eta}\left(B^{\pi}(x, r)\right)}=-\mathbf{I}_{m}\left(\xi \mid \hat{\eta} \vee \pi^{-1} \gamma\right)(x),
$$

where $\mathbf{I}_{m}(\cdot \mid \cdot)$ denotes the conditional information (see (2.2) for the definition). Furthermore, set

$$
g(x)=-\inf _{r>0} \log \frac{m_{x}^{\eta}\left(B^{\pi}(x, r) \cap \xi(x)\right)}{m_{x}^{\eta}\left(B^{\pi}(x, r)\right)}
$$

and assume $H_{m}(\xi)<\infty$. Then $g \geq 0$ and $g \in L^{1}(X, \mathcal{B}, m)$.

PROOF: (3.5) follows directly from Lemma 3.3 and the following equality:

$$
\lim _{r \rightarrow 0} \log \frac{m_{x}^{\eta}\left(B^{\pi}(x, r) \cap \xi(x)\right)}{m_{x}^{\eta}\left(B^{\pi}(x, r)\right)}=\sum_{A \in \xi} \chi_{A}(x) \lim _{r \rightarrow 0} \log \frac{m_{x}^{\eta}\left(B^{\pi}(x, r) \cap A\right)}{m_{x}^{\eta}\left(B^{\pi}(x, r)\right)} .
$$

Now we turn to the proof of (3.6). It is clear that $g$ is nonnegative. By Lemma 3.2, $g$ is measurable. In the following we show that $g \in L^{1}(X, \mathcal{B}, m)$.

Let $C \in \eta$ and $A \in \xi$ be given. As in the proof of Lemma 3.3, we define measures $\mu_{C}$ and $\nu_{C}$ on $\mathbb{R}^{d}$ by $\mu_{C}(E)=m_{C}\left(\pi^{-1} E \cap A\right)$ and $\nu_{C}(E)=m_{C}\left(\pi^{-1} E\right)$ for all $E \in \gamma$. By theorem 7.4 in [56], we have

$$
\mu_{C}\left\{z \in \mathbb{R}^{d}: \inf _{r>0} \frac{\mu_{C}(B(z, r))}{v_{C}(B(z, r))}<\lambda\right\} \leq 3^{d} \lambda, \quad \lambda>0 .
$$

Hence for any $\lambda>0$,

$$
m_{C}\left(\left\{x \in X: \inf _{r>0} \frac{m_{C}\left(B^{\pi}(x, r) \cap A\right)}{m_{C}\left(B^{\pi}(x, r)\right)}<\lambda\right\} \cap A\right) \leq 3^{d} \lambda .
$$

Integrating $C$ with respect to $m_{\eta}$, we obtain

$$
m\left(\left\{x \in X: \inf _{r>0} \frac{m_{x}^{\eta}\left(B^{\pi}(x, r) \cap A\right)}{m_{x}^{\eta}\left(B^{\pi}(x, r)\right)}<\lambda\right\} \cap A\right) \leq 3^{d} \lambda
$$


Denote

$$
g^{A}(x)=\inf _{r>0} \frac{m_{x}^{\eta}\left(B^{\pi}(x, r) \cap A\right)}{m_{x}^{\eta}\left(B^{\pi}(x, r)\right)} .
$$

Then the above inequality can be rewritten as

$$
m\left(A \cap\left\{g^{A}<\lambda\right\}\right) \leq 3^{d} \lambda .
$$

Note that by (3.6), $g(x)=-\sum_{A \in \xi} \chi_{A}(x) \log g^{A}(x)$. Since $g$ is nonnegative, we have

$$
\begin{aligned}
\int g d m=\int_{0}^{\infty} m\{g>t\} d t & =\int_{0}^{\infty} \sum_{A \in \xi} m\left(A \cap\left\{g^{A}<e^{-t}\right\}\right) d t \\
& \leq \sum_{A \in \xi} \int_{0}^{\infty} \min \left\{m(A), 3^{d} e^{-t}\right\} d t \\
& \leq \sum_{A \in \xi}\left(-m(A) \log m(A)+m(A)+m(A) \log 3^{d}\right) \\
& =H_{m}(\xi)+1+\log 3^{d} .
\end{aligned}
$$

This finishes the proof of the proposition.

Remark 3.6. Consider the case $X=\Sigma$ and $\xi=\mathcal{P}$, where $\mathcal{P}$ is defined as in (2.3). Suppose that $\left\{S_{i}\right\}_{i=1}^{\ell}$ is a family of mappings such that $S_{i}: \pi(\Sigma) \rightarrow S_{i}(\pi(\Sigma)) \subset$ $\mathbb{R}^{d}$ is homeomorphic for each $i$. Then in (3.5) and (3.6), we can change the terms $B^{\pi}(x, r)$ to $\pi^{-1} R_{r, x}(\pi x)$, where $R_{r, x}(z):=S_{x_{1}}^{-1} B\left(S_{x_{1}}(z), r\right)$. To see this, fix $i$ and define $\pi^{\prime}=S_{i} \circ \pi$. Then we have

$$
\begin{aligned}
\lim _{r \rightarrow 0} \frac{m_{x}^{\eta}\left(\pi^{-1} R_{r, x}(\pi x) \cap[i]\right)}{m_{x}^{\eta}\left(\pi^{-1} R_{r, x}(\pi x)\right)} & =\lim _{r \rightarrow 0} \frac{m_{x}^{\eta}\left(B^{\pi^{\prime}}(x, r) \cap[i]\right)}{m_{x}^{\eta}\left(B^{\pi^{\prime}}(x, r)\right)} \\
& =\mathbf{E}_{m}\left(\chi[i] \mid \hat{\eta} \vee\left(\pi^{\prime}\right)^{-1} \gamma\right)(x) .
\end{aligned}
$$

However, $\left(\pi^{\prime}\right)^{-1} \gamma=\pi^{-1} \gamma$ due to the assumption on $S_{i}$. Hence the last term in the above formula equals $\mathbf{E}_{m}\left(\chi_{[i]} \mid \hat{\eta} \vee \pi^{-1} \gamma\right)(x)$. Thus we can replace the terms $B^{\pi}(x, r)$ by $\pi^{-1} R_{r, x}(\pi x)$ in (3.5). For the change in (3.6), we may use a similar argument.

Lemma 3.7 Let $\pi: X \rightarrow \mathbb{R}^{d}$ and $\phi: X \rightarrow \mathbb{R}^{k}$ be two $\mathcal{B}$-measurable maps. Let $\eta$ be the partition of $X$ given by $\eta=\left\{\pi^{-1}(z): z \in \mathbb{R}^{d}\right\}$. Let $A \in \mathcal{B}$ and $t>0$. Then for $m$-a.e. $x \in X$, we have

$$
m_{x}^{\eta}\left(B^{\phi}(x, t) \cap A\right) \geq \limsup _{r \rightarrow 0} \frac{m\left(B^{\phi}(x, t) \cap A \cap B^{\pi}(x, r)\right)}{m\left(B^{\pi}(x, r)\right)}
$$

and

$$
m_{x}^{\eta}\left(U^{\phi}(x, t) \cap A\right) \leq \liminf _{r \rightarrow 0} \frac{m\left(U^{\phi}(x, t) \cap A \cap B^{\pi}(x, r)\right)}{m\left(B^{\pi}(x, r)\right)},
$$


where $B^{\phi}(x, t):=\phi^{-1} B(\phi x, t)$ and $U^{\phi}(x, t):=\phi^{-1} U(\phi x, t)$; here $U(z, t)$ denotes the open ball in $\mathbb{R}^{k}$ centered at $z$ of radius $t$.

Proof: Fix $A \in \mathcal{B}$ and $t>0$. Similar to the proof of Lemma 3.2, for $n \in \mathbb{N}$, let $\mathcal{D}_{n}$ denote the collection

$$
\mathcal{D}_{n}=\left\{\left[0,2^{-n}\right)^{k}+\alpha: \alpha \in 2^{-n} \mathbb{Z}^{k}\right\} .
$$

For $y \in \mathbb{R}^{k}$, denote

$$
W_{n}(y)=\bigcup_{Q \in \mathcal{D}_{n}: Q \cap B(y, t) \neq \varnothing} Q, \quad \hat{W}_{n}(y)=\bigcup_{Q \in \mathcal{D}_{n}: Q \subset U(y, t)} Q .
$$

Write $\mathcal{W}_{n}:=\left\{W_{n}(y): y \in \mathbb{R}^{k}\right\}$ and $\widehat{\mathcal{W}}_{n}:=\left\{\widehat{W}_{n}(y): y \in \mathbb{R}^{k}\right\}$. It is clear that both $\mathcal{W}_{n}$ and $\widehat{\mathcal{W}}_{n}$ are countable for each $n \in \mathbb{N}$. Furthermore, we have $W_{n}(y) \downarrow$ $B(y, t)$ and $\hat{W}_{n}(y) \uparrow U(y, t)$ for each $y \in \mathbb{R}^{k}$ as $n \rightarrow \infty$. As a consequence, we have $\phi^{-1} W_{n}(\phi x) \downarrow B^{\phi}(x, t)$ and $\phi^{-1} \widehat{W}_{n}(\phi x) \uparrow U^{\phi}(x, t)$ for $x \in X$. Therefore

$$
m_{x}^{\eta}\left(B^{\phi}(x, t) \cap A\right)=\lim _{n \rightarrow \infty} m_{x}^{\eta}\left(\phi^{-1} W_{n}(\phi x) \cap A\right)
$$

and

$$
m_{x}^{\eta}\left(U^{\phi}(x, t) \cap A\right)=\lim _{n \rightarrow \infty} m_{x}^{\eta}\left(\phi^{-1} \widehat{W}_{n}(\phi x) \cap A\right)
$$

for each $x \in X$.

In the following we only prove (3.7). The proof of (3.8) is essentially identical. For $n \in \mathbb{N}$ and $F \in \mathcal{W}_{n}$, let $\Gamma_{n}(F)=\left\{x \in X: W_{n}(\phi x)=F\right\}$. Then for $m$-a.e. $x$ and all $n \in \mathbb{N}$, we have

$$
\begin{aligned}
m_{x}^{\eta} & \left(\phi^{-1} W_{n}(\phi x) \cap A\right) \\
& =\sum_{F \in \mathcal{W}_{n}} \chi_{\Gamma_{n}(F)}(x) m_{x}^{\eta}\left(\phi^{-1} F \cap A\right) \\
& =\sum_{F \in \mathcal{W}_{n}} \chi_{\Gamma_{n}(F)}(x) \mathbf{E}_{m}\left(\chi_{\phi^{-1} F \cap A} \mid \hat{\eta}\right)(x) \\
& =\sum_{F \in \mathcal{W}_{n}} \chi_{\Gamma_{n}(F)}(x) \mathbf{E}_{m}\left(\chi_{\phi^{-1} F \cap A} \mid \pi^{-1} \gamma\right)(x) \\
& =\sum_{F \in \mathcal{W}_{n}} \chi_{\Gamma_{n}(F)}(x) \lim _{r \rightarrow 0} \frac{m\left(\phi^{-1} F \cap A \cap B^{\pi}(x, r)\right)}{m\left(B^{\pi}(x, r)\right)} \quad \text { (by Lemma 3.3) } \\
& =\lim _{r \rightarrow 0} \frac{m\left(\phi^{-1} W_{n}(\phi x) \cap A \cap B^{\pi}(x, r)\right)}{m\left(B^{\pi}(x, r)\right)} \\
& \geq \limsup _{r \rightarrow 0} \frac{m\left(B^{\phi}(x, t) \cap A \cap B^{\pi}(x, r)\right)}{m\left(B^{\pi}(x, r)\right)} .
\end{aligned}
$$

Letting $n \rightarrow \infty$, we obtain (3.7). 
Remark 3.8. Under the conditions of Lemma 3.7, assume that

$$
g: \pi(X) \rightarrow g(\pi(X)) \subset \mathbb{R}^{d}
$$

is a homeomorphism. Then we may replace the terms $B^{\pi}(x, r)$ in (3.7) and (3.8) by $B^{g \pi}(x, r)$. To see this, let $\pi^{\prime}=g \circ \pi$. It is easy to see the partition $\eta$ is just the same as $\left\{\left(\pi^{\prime}\right)^{-1}(z): z \in \mathbb{R}^{d}\right\}$.

Proposition 3.9 Let $T: X \rightarrow X$ be a measure-preserving transformation on $(X, \mathcal{B}, m)$, and let $\eta$ be a measurable partition of $X$. Suppose that $\pi: X \rightarrow \mathbb{R}^{d}$ is a bounded $\mathcal{B}$-measurable function. Then for any $r>0$,

$$
\lim _{n \rightarrow \infty} \frac{1}{n} \log m_{T^{n} x}^{\eta}\left(B^{\pi}\left(T^{n} x, r\right)\right)=0 \quad \text { for } m \text {-a.e. } x \in X .
$$

Proof: Fix $r>0$ and $t>0$. Since $\pi(X)$ is a bounded subset of $\mathbb{R}^{d}$, we can cover it by $\ell$ balls $B\left(\pi x_{i}, \frac{r}{2}\right)$ of radius $\frac{r}{2}$, where $x_{i} \in X$ and $i=1, \ldots, \ell$. Define

$$
A_{n}=\left\{x \in X: m_{x}^{\eta}\left(B^{\pi}(x, r)\right) \leq e^{-n t}\right\}, \quad n \in \mathbb{N} .
$$

If a ball $B^{\pi}\left(x_{i}, \frac{r}{2}\right)$ intersects $A_{n}$, then for any $y \in A_{n} \cap B^{\pi}\left(x_{i}, \frac{r}{2}\right)$, we have $B^{\pi}\left(x_{i}, \frac{r}{2}\right) \subset B^{\pi}(y, r)$ because $B\left(\pi x_{i}, \frac{r}{2}\right) \subset B(\pi y, r)$ by the triangle inequality. So the definition of $A_{n}$ gives $m_{y}^{\eta}\left(A_{n} \cap B^{\pi}\left(x_{i}, \frac{r}{2}\right)\right) \leq m_{y}^{\eta}\left(B^{\pi}(y, r)\right) \leq e^{-n t}$. Hence

$$
m\left(A_{n} \cap B^{\pi}\left(x_{i}, \frac{r}{2}\right)\right)=\int m_{y}^{\eta}\left(A_{n} \cap B^{\pi}\left(x_{i}, \frac{r}{2}\right)\right) d m(y) \leq e^{-n t}
$$

and $m\left(A_{n}\right) \leq \ell e^{-n t}$.

This estimate gives directly that $g(x):=\log m_{x}^{\eta}\left(B^{\pi}(x, r)\right) \in L^{1}(X, \mathcal{B}, m)$. Note that $g\left(T^{n} x\right)=\sum_{i=1}^{n} g\left(T^{i} x\right)-\sum_{i=1}^{n-1} g\left(T^{i} x\right)$. By the Birkhoff ergodic theorem we can get $\lim _{n \rightarrow \infty} \frac{1}{n} g\left(T^{n} x\right)=0$ for $m$-a.e. $x \in X$, which is the desired result.

Lemma 3.10 Let $\mathcal{A}$ be a sub- $\sigma$-algebra of $\mathcal{B}$. Let $A \in \mathcal{B}$ with $m(A)>0$. Then

$$
\mathbf{E}_{m}\left(\chi_{A} \mid \mathcal{A}\right)(x)>0
$$

for m-a.e. $x \in A$.

Proof: Let $W:=\left\{\mathbf{E}_{m}\left(\chi_{A} \mid \mathcal{A}\right) \leq 0\right\}$. Then $W \in \mathcal{A}$. Hence

$$
0 \geq \int_{W} \mathbf{E}_{m}\left(\chi_{A} \mid \mathcal{A}\right) d m=\int_{W} \chi_{A} d m(x)=m(A \cap W),
$$

which implies $m(A \cap W)=0$. This finishes the proof.

\section{Projection Measure-Theoretic Entropies Associated with IFSs}

Throughout this section, let $\left\{S_{i}\right\}_{i=1}^{\ell}$ be an IFS on a closed set $X \subset \mathbb{R}^{d}$, and $(\Sigma, \sigma)$ the one-sided full shift over $\{1, \ldots, \ell\}$. Let $\mathcal{M}_{\sigma}(\Sigma)$ denote the collection of all $\sigma$-invariant Borel probability measures on $\Sigma$. Let $\pi: \Sigma \rightarrow \mathbb{R}^{d}$ be defined as in (2.1), and $h_{\pi}(\sigma, \cdot)$ as in Definition 2.1. 


\subsection{Some Basic Properties}

In this subsection, we present some basic properties of projection measuretheoretic entropy. Our first result is the following:

\section{PROPOSITION 4.1}

(i) $0 \leq h_{\pi}(\sigma, m) \leq h(\sigma, m)$ for every $m \in \mathcal{M}_{\sigma}(\Sigma)$, where $h(\sigma, m)$ denotes the classical measure-theoretic entropy of $m$.

(ii) The projection entropy function is affine on $\mathcal{M}_{\sigma}(\Sigma)$, i.e., for any $m_{1}, m_{2} \in$ $\mathcal{M}_{\sigma}(\Sigma)$ and any $0 \leq p \leq 1$, we have

$$
h_{\pi}\left(\sigma, p m_{1}+(1-p) m_{2}\right)=p h_{\pi}\left(\sigma, m_{1}\right)+(1-p) h_{\pi}\left(\sigma, m_{2}\right) .
$$

The proof of the above proposition will be given later. Now let us recall some notation. If $\xi$ is a partition of $\Sigma$, then $\hat{\xi}$ denotes the $\sigma$-algebra generated by $\xi$. If $\xi_{1}, \ldots, \xi_{n}$ are countable partitions of $\Sigma$, then $\bigvee_{i=1}^{n} \xi_{i}$ denotes the partition consisting of sets $A_{1} \cap \cdots \cap A_{n}$ with $A_{i} \in \xi_{i}$. Similarly for $\sigma$-algebras $\mathcal{A}_{1}, \mathcal{A}_{2}, \ldots$, $\bigvee_{n} \mathcal{A}_{n}$ denotes the $\sigma$-algebra generated by $\bigcup_{n} \mathcal{A}_{n}$.

Let $\mathcal{P}$ be the partition of $\Sigma$ defined as in (2.3). Write $\mathcal{P}_{0}^{n}=\bigvee_{i=0}^{n} \sigma^{-i} \mathcal{P}$ for $n \geq 0$. Let $\gamma$ denote the Borel $\sigma$-algebra $\mathcal{B}\left(\mathbb{R}^{d}\right)$ on $\mathbb{R}^{d}$. Similar to Definition 2.1, we give the following definition:

Definition 4.2 Let $k \in \mathbb{N}$ and $v \in \mathcal{M}_{\sigma^{k}}(\Sigma)$. Define

$$
h_{\pi}\left(\sigma^{k}, v\right):=H_{\nu}\left(\mathcal{P}_{0}^{k-1} \mid \sigma^{-k} \pi^{-1} \gamma\right)-H_{v}\left(\mathcal{P}_{0}^{k-1} \mid \pi^{-1} \gamma\right) .
$$

The term $h_{\pi}\left(\sigma^{k}, v\right)$ can be viewed as the projection measure-theoretic entropy of $v$ with respect to the IFS $\left\{S_{i_{1}} \circ \cdots \circ S_{i_{k}}: 1 \leq i_{j} \leq \ell\right.$ for $\left.1 \leq j \leq k\right\}$. The following proposition exploits the connection between $h_{\pi}\left(\sigma^{k}, v\right)$ and $h_{\pi}(\sigma, m)$, where $m=\frac{1}{k} \sum_{i=0}^{k-1} v \circ \sigma^{-i}$.

Proposition 4.3 Let $k \in \mathbb{N}$ and $v \in \mathcal{M}_{\sigma^{k}}(\Sigma)$. Set $m=\frac{1}{k} \sum_{i=0}^{k-1} v \circ \sigma^{-i}$. Then $m$ is $\sigma$-invariant, and $h_{\pi}(\sigma, m)=\frac{1}{k} h_{\pi}\left(\sigma^{k}, v\right)$.

To prove Propositions 4.1 and 4.3, we first give some lemmas about the (conditional) information and entropy (see Section 2 for the definitions).

LEMMA 4.4 (cf. [48]) Let $m$ be a Borel probability measure on $\Sigma$. Let $\xi, \eta$ be two countable Borel partitions of $\Sigma$ with $H_{m}(\xi)<\infty, H_{m}(\eta)<\infty$, and $\mathcal{A}$ a sub- $\sigma$-algebra of $\mathcal{B}(\Sigma)$. Then we have the following:

(i) $\mathbf{I}_{m \circ \sigma^{-1}}(\xi \mid \mathcal{A}) \circ \sigma=\mathbf{I}_{m}\left(\sigma^{-1} \xi \mid \sigma^{-1} \mathcal{A}\right)$.

(ii) $\mathbf{I}_{m}(\xi \vee \eta \mid \mathcal{A})=\mathbf{I}_{m}(\xi \mid \mathcal{A})+\mathbf{I}_{m}(\eta \mid \hat{\xi} \vee \mathcal{A})$.

(iii) $H_{m}(\xi \vee \eta \mid \mathcal{A})=H_{m}(\xi \mid \mathcal{A})+H(\eta \mid \hat{\xi} \vee \mathcal{A})$.

(iv) If $\mathcal{A}_{1} \subset \mathcal{A}_{2} \subset \cdots$ is an increasing sequence of sub- $\sigma$-algebras with $\mathcal{A}_{n} \uparrow$ $\mathcal{A}$, then $\mathbf{I}_{m}\left(\xi \mid \mathcal{A}_{n}\right)$ converges almost everywhere and in $L^{1}$ to $\mathbf{I}_{m}(\xi \mid \mathcal{A})$. In particular, $\lim _{n \rightarrow \infty} H_{m}\left(\xi \mid \mathcal{A}_{n}\right)=H_{m}(\xi \mid \mathcal{A})$. 
Lemma 4.5 Denote $g(x)=-x \log x$ for $x \geq 0$. For any integer $k \geq 2$ and $x_{1}, \ldots, x_{k} \geq 0$, we have

$$
\frac{1}{k} \sum_{i=1}^{k} g\left(x_{i}\right) \leq g\left(\frac{1}{k} \sum_{i=1}^{k} x_{i}\right) \leq \sum_{i=1}^{k} g\left(\frac{x_{i}}{k}\right)
$$

and

$$
\sum_{i=1}^{k} g\left(x_{i}\right)-\left(x_{1}+\cdots+x_{k}\right) \log k \leq g\left(x_{1}+\cdots+x_{k}\right) \leq \sum_{i=1}^{k} g\left(x_{i}\right) .
$$

Moreover, for any $p_{1}, p_{2} \geq 0$ with $p_{1}+p_{2}=1$,

$$
\sum_{j=1}^{2} p_{j} g\left(x_{j}\right) \leq g\left(\sum_{j=1}^{2} p_{j} x_{j}\right) \leq \sum_{j=1}^{2} p_{j} g\left(x_{j}\right)+g\left(p_{j}\right) x_{j}
$$

PROOF: The proof is standard.

LEMMA 4.6 Let $m$ be a Borel probability measure on $\Sigma$. Assume $\xi$ and $\eta$ are two countable Borel partitions of $\Sigma$ such that each member in $\xi$ intersects at most $k$ members of $\eta$. Then $H_{m}(\xi) \geq H_{m}(\xi \vee \eta)-\log k$.

PROOF: Although the result is standard, we give a short proof for the convenience of the reader. Denote $g(x)=-x \log x$ for $x \in[0,1]$. Then

$$
\begin{aligned}
H_{m}(\xi) & =\sum_{A \in \xi} g(m(A))=\sum_{A \in \xi} g\left(\sum_{B \in \eta, B \cap A \neq \varnothing} m(A \cap B)\right) \\
& \geq \sum_{A \in \xi}\left[\left(\sum_{B \in \eta, B \cap A \neq \varnothing} g(m(A \cap B))\right)-m(A) \log k\right] \\
& \geq\left(\sum_{A \in \xi} \sum_{B \in \eta} g(m(A \cap B))\right)-\log k \\
& =H_{m}(\xi \vee \eta)-\log k .
\end{aligned}
$$

This finishes the proof.

The following simple lemma plays an important role in our analysis.

Lemma 4.7 $\hat{\mathcal{P}} \vee \sigma^{-1} \pi^{-1} \gamma=\hat{\mathcal{P}} \vee \pi^{-1} \gamma$.

Proof: We only prove $\hat{\mathcal{P}} \vee \sigma^{-1} \pi^{-1} \gamma \subseteq \hat{\mathcal{P}} \vee \pi^{-1} \gamma$. The other direction can be proved by an essentially identical argument. Note that each member in $\widehat{\mathcal{P}} \vee \sigma^{-1} \pi^{-1} \gamma$ can be written as

$$
\bigcup_{j=1}^{\ell}[j] \cap \sigma^{-1} \pi^{-1} A_{j}
$$


with $A_{j} \in \gamma$. However, it is direct to check that

$$
[j] \cap \sigma^{-1} \pi^{-1} A_{j}=[j] \cap \pi^{-1}\left(S_{j}\left(A_{j}\right)\right) .
$$

Since $S_{j}$ is injective and contractive (thus continuous), we have $S_{j}\left(A_{j}\right) \in \gamma$. Therefore $\bigcup_{j=1}^{\ell}[j] \cap \sigma^{-1} \pi^{-1} A_{j} \in \hat{\mathcal{P}} \vee \pi^{-1} \gamma$.

LEMMA 4.8 Let $m$ be a Borel probability measure on $\Sigma$ and $k \in \mathbb{N}$. We have

$$
\begin{aligned}
H_{m}\left(\mathcal{P}_{0}^{k-1} \mid \sigma^{-k} \pi^{-1} \gamma\right)- & H_{m}\left(\mathcal{P}_{0}^{k-1} \mid \pi^{-1} \gamma\right)= \\
& \sum_{j=0}^{k-1} H_{m \circ \sigma^{-j}}\left(\mathcal{P} \mid \sigma^{-1} \pi^{-1} \gamma\right)-H_{m \circ \sigma^{-j}}\left(\mathcal{P} \mid \pi^{-1} \gamma\right) .
\end{aligned}
$$

Moreover, if $m \in \mathcal{M}_{\sigma}(\Sigma)$, then

$$
H_{m}\left(\mathcal{P}_{0}^{k-1} \mid \sigma^{-k} \pi^{-1} \gamma\right)-H_{m}\left(\mathcal{P}_{0}^{k-1} \mid \pi^{-1} \gamma\right)=k h_{\pi}(\sigma, m) .
$$

Proof: For $j=0,1, \ldots, k-1$, we have

$$
\begin{aligned}
\mathbf{I}_{m} & \left(\mathcal{P}_{0}^{k-1} \mid \sigma^{-j} \pi^{-1} \gamma\right)-\mathbf{I}_{m}\left(\mathcal{P}_{0}^{k-1} \mid \sigma^{-(j+1)} \pi^{-1} \gamma\right) \\
= & \mathbf{I}_{m}\left(\sigma^{-j} \mathcal{P} \mid \sigma^{-j} \pi^{-1} \gamma\right)+\mathbf{I}_{m}\left(\bigvee_{\substack{0 \leq i \leq k-1, i \neq j}} \sigma^{-i} \mathcal{P} \mid \sigma^{-j} \hat{\mathcal{P}} \vee \sigma^{-j} \pi^{-1} \gamma\right) \\
& -\mathbf{I}_{m}\left(\mathcal{P}_{0}^{k-1} \mid \sigma^{-(j+1)} \pi^{-1} \gamma\right) \quad(\text { by Lemma 4.4(ii)) } \\
= & \mathbf{I}_{m}\left(\sigma^{-j} \mathcal{P} \mid \sigma^{-j} \pi^{-1} \gamma\right)+\mathbf{I}_{m}\left(\bigvee_{\substack{0 \leq i \leq k-1 \\
i \neq j}} \sigma^{-i} \mathcal{P} \mid \sigma^{-j} \hat{\mathcal{P}} \vee \sigma^{-(j+1)} \pi^{-1} \gamma\right) \\
& -\mathbf{I}_{m}\left(\mathcal{P}_{0}^{k-1} \mid \sigma^{-(j+1)} \pi^{-1} \gamma\right) \quad(\text { by Lemma 4.7) } \\
= & \mathbf{I}_{m}\left(\sigma^{-j} \mathcal{P} \mid \sigma^{-j} \pi^{-1} \gamma\right)-\mathbf{I}_{m}\left(\sigma^{-j} \mathcal{P} \mid \sigma^{-(j+1)} \pi^{-1} \gamma\right) \quad \text { (by Lemma 4.4(ii)) } \\
= & \mathbf{I}_{m \circ \sigma^{-j}}\left(\mathcal{P} \mid \pi^{-1} \gamma\right) \circ \sigma^{j}-\mathbf{I}_{m \circ \sigma^{-j}}\left(\mathcal{P} \mid \sigma^{-1} \pi^{-1} \gamma\right) \circ \sigma^{j} \quad \text { (by Lemma 4.4(i)). }
\end{aligned}
$$

Summing $j$ over $\{0, \ldots, k-1\}$ yields

$$
\begin{aligned}
\mathbf{I}_{m}\left(\mathcal{P}_{0}^{k-1} \mid \pi^{-1} \gamma\right)-\mathbf{I}_{m}\left(\mathcal{P}_{0}^{k-1} \mid \sigma^{-k} \pi^{-1} \gamma\right) & = \\
& \sum_{j=0}^{k-1}\left(\mathbf{I}_{m \circ \sigma^{-j}}\left(\mathcal{P} \mid \pi^{-1} \gamma\right) \circ \sigma^{j}-\mathbf{I}_{m \circ \sigma^{-j}}\left(\mathcal{P} \mid \sigma^{-1} \pi^{-1} \gamma\right) \circ \sigma^{j}\right) .
\end{aligned}
$$

Integrating, we obtain the desired formula.

For any $n \in \mathbb{N}$, let $\mathcal{D}_{n}$ be the partition of $\mathbb{R}^{d}$ given by

$$
\mathcal{D}_{n}=\left\{\left[0,2^{-n}\right)^{d}+\alpha: \alpha \in 2^{-n} \mathbb{Z}^{d}\right\} .
$$

LEMMA 4.9 Let $m \in \mathcal{M}_{\sigma}(\Sigma)$. For each $n \in \mathbb{N}$, we have

$$
H_{m}\left(\mathcal{P} \mid \sigma^{-1} \pi^{-1} \hat{\mathcal{D}_{n}}\right)-H_{m}\left(\mathcal{P} \mid \pi^{-1} \hat{\mathcal{D}_{n}}\right) \geq-d \log (\sqrt{d}+1) .
$$


PROOF: Since $m$ is $\sigma$-invariant, by Lemma 4.4(iii), we have

$$
\begin{aligned}
H_{m}\left(\mathcal{P} \mid \sigma^{-1} \pi^{-1} \hat{\mathcal{D}_{n}}\right)-H_{m}\left(\mathcal{P} \mid \pi^{-1} \hat{\mathcal{D}_{n}}\right) \\
=H_{m}\left(\mathcal{P} \vee \sigma^{-1} \pi^{-1} \mathcal{D}_{n}\right)-H_{m}\left(\sigma^{-1} \pi^{-1} \mathcal{D}_{n}\right) \\
\quad-H_{m}\left(\mathcal{P} \vee \pi^{-1} \mathcal{D}_{n}\right)+H_{m}\left(\pi^{-1} \mathcal{D}_{n}\right) \\
=H_{m}\left(\mathcal{P} \vee \sigma^{-1} \pi^{-1} \mathcal{D}_{n}\right)-H_{m}\left(\mathcal{P} \vee \pi^{-1} \mathcal{D}_{n}\right) .
\end{aligned}
$$

Observe that for each $1 \leq j \leq \ell$ and $Q \in \mathcal{D}_{n}$,

$$
[j] \cap \sigma^{-1} \pi^{-1}(Q)=[j] \cap \pi^{-1}\left(S_{j}(Q)\right) .
$$

Since $S_{j}$ is contractive, $\operatorname{diam}\left(S_{j}(Q)\right) \leq 2^{-n} \sqrt{d}$ and thus $S_{j}(Q)$ intersects at most $(\sqrt{d}+1)^{d}$ members in $\mathcal{D}_{n}$. We deduce that $[j] \cap \sigma^{-1} \pi^{-1}(Q)$ intersects at most $(\sqrt{d}+1)^{d}$ members in $\mathcal{P} \vee \pi^{-1} \mathcal{D}_{n}$. By Lemma 4.6, we have

$$
\begin{aligned}
H_{m}\left(\mathcal{P} \vee \sigma^{-1} \pi^{-1} \mathcal{D}_{n}\right) \geq & H_{m}\left(\mathcal{P} \vee \sigma^{-1} \pi^{-1} \mathcal{D}_{n} \vee \pi^{-1} \mathcal{D}_{n}\right) \\
& -d \log (\sqrt{d}+1) \\
\geq & H_{m}\left(\mathcal{P} \vee \pi^{-1} \mathcal{D}_{n}\right)-d \log (\sqrt{d}+1) .
\end{aligned}
$$

Combining this with (4.6) yields the desired inequality.

Proof OF Proposition 4.1: We first prove part (i) of the proposition, i.e.,

$$
0 \leq h_{\pi}(\sigma, m) \leq h(\sigma, m) .
$$

Since $\hat{\mathcal{D}_{n}} \uparrow \gamma$ as $n$ tends to $\infty$, by Lemma 4.4(iv), we have

$$
\begin{aligned}
& \lim _{n \rightarrow \infty} H_{m}\left(\mathcal{P} \mid \sigma^{-1} \pi^{-1} \hat{\mathcal{D}}_{n}\right)-H_{m}\left(\mathcal{P} \mid \pi^{-1} \hat{\mathcal{D}}_{n}\right) \\
& H_{m}\left(\mathcal{P} \mid \sigma^{-1} \pi^{-1} \gamma\right)-H_{m}\left(\mathcal{P} \mid \pi^{-1} \gamma\right) .
\end{aligned}
$$

This together with Lemma 4.9 yields

$$
H_{m}\left(\mathcal{P} \mid \sigma^{-1} \pi^{-1} \gamma\right)-H_{m}\left(\mathcal{P} \mid \pi^{-1} \gamma\right) \geq-d \log (\sqrt{d}+1) .
$$

Applying the same argument to the IFS $\left\{S_{i_{1} \cdots i_{k}}: 1 \leq i_{j} \leq \ell, 1 \leq j \leq k\right\}$, we have

$$
H_{m}\left(\mathcal{P}_{0}^{k-1} \mid \sigma^{-k} \pi^{-1} \gamma\right)-H_{m}\left(\mathcal{P}_{0}^{k-1} \mid \pi^{-1} \gamma\right) \geq-d \log (\sqrt{d}+1) .
$$

This together with Lemma 4.8 yields $h_{\pi}(\sigma, m) \geq-d \log (\sqrt{d}+1) / k$. Since $k$ is arbitrary, we have $h_{\pi}(\sigma, m) \geq 0$. To see $h_{\pi}(\sigma, m) \leq h(\sigma, m)$, it suffices to observe that

$$
\begin{aligned}
k h_{\pi}(\sigma, m) & =H_{m}\left(\mathcal{P}_{0}^{k-1} \mid \sigma^{-k} \pi^{-1} \gamma\right)-H_{m}\left(\mathcal{P}_{0}^{k-1} \mid \pi^{-1} \gamma\right) \\
& \leq H_{m}\left(\mathcal{P}_{0}^{k-1} \mid \sigma^{-k} \pi^{-1} \gamma\right) \leq H_{m}\left(\mathcal{P}_{0}^{k-1}\right)
\end{aligned}
$$


Now we turn to the proof of part (ii). Let $m_{1}, m_{2} \in \mathcal{M}_{\sigma}(\Sigma)$ and $m=p m_{1}+$ $(1-p) m_{2}$ for some $p \in[0,1]$. Using (4.3), for any finite or countable Borel partition $\xi$ we have

$$
\left|H_{m}(\xi)-p H_{m_{1}}(\xi)-(1-p) H_{m_{2}}(\xi)\right| \leq g(p)+g(1-p) \leq \log 2 .
$$

Let $k \in \mathbb{N}$. By Lemma 4.8, Lemma 4.4(iv), and (4.6), we have

$$
\begin{aligned}
h_{\pi}(\sigma, m) & =\frac{1}{k}\left(H_{m}\left(\mathcal{P}_{0}^{k-1} \mid \sigma^{-k} \pi^{-1} \gamma\right)-H_{m}\left(\mathcal{P}_{0}^{k-1} \mid \pi^{-1} \gamma\right)\right) \\
& =\frac{1}{k} \lim _{n \rightarrow \infty}\left(H_{m}\left(\mathcal{P}_{0}^{k-1} \mid \sigma^{-k} \pi^{-1} \widehat{\mathcal{D}_{n}}\right)-H_{m}\left(\mathcal{P}_{0}^{k-1} \mid \pi^{-1} \widehat{\mathcal{D}_{n}}\right)\right) \\
& =\frac{1}{k} \lim _{n \rightarrow \infty}\left(H_{m}\left(\mathcal{P}_{0}^{k-1} \vee \sigma^{-k} \pi^{-1} \mathcal{D}_{n}\right)-H_{m}\left(\mathcal{P}_{0}^{k-1} \vee \pi^{-1} \mathcal{D}_{n}\right)\right)
\end{aligned}
$$

The above statement is true when $m$ is replaced by $m_{1}$ and $m_{2}$. However, by (4.8),

$$
H_{m}\left(\mathcal{P}_{0}^{k-1} \vee \sigma^{-k} \pi^{-1} \mathcal{D}_{n}\right)-H_{m}\left(\mathcal{P}_{0}^{k-1} \vee \pi^{-1} \mathcal{D}_{n}\right)
$$

differs from

$$
\sum_{j=1}^{2} p_{j}\left[H_{m_{j}}\left(\mathcal{P}_{0}^{k-1} \vee \sigma^{-k} \pi^{-1} \mathcal{D}_{n}\right)-H_{m_{j}}\left(\mathcal{P}_{0}^{k-1} \vee \pi^{-1} \mathcal{D}_{n}\right)\right]
$$

at most $2 \log 2$, where $p_{1}=p$ and $p_{2}=1-p$. This together with (4.9) yields (4.1).

Proof of Proposition 4.3: Let $k \geq 2$ and $v \in \mathcal{M}_{\sigma^{k}}(\Sigma)$. We claim that $h_{\pi}\left(\sigma^{k}, v \circ \sigma^{-j}\right)=h_{\pi}\left(\sigma^{k}, v\right)$ for any $1 \leq j \leq k-1$. To prove the claim, it suffices to prove $h_{\pi}\left(\sigma^{k}, v \circ \sigma^{-1}\right)=h_{\pi}\left(\sigma^{k}, v\right)$. Note that both $v$ and $v \circ \sigma^{-1}$ are $\sigma^{k}$-invariant. By Lemma 4.8, we have

$$
\begin{aligned}
h_{\pi}\left(\sigma^{k}, \nu\right) & =H_{\nu}\left(\mathcal{P}_{0}^{k-1} \mid \sigma^{-k} \pi^{-1} \gamma\right)-H_{\nu}\left(\mathcal{P}_{0}^{k-1} \mid \pi^{-1} \gamma\right) \\
& =\sum_{j=0}^{k-1}\left(H_{\nu \circ \sigma^{-j}}\left(\mathcal{P} \mid \sigma^{-1} \pi^{-1} \gamma\right)-H_{\nu \circ \sigma^{-j}}\left(\mathcal{P} \mid \pi^{-1} \gamma\right)\right),
\end{aligned}
$$

while

$$
\begin{aligned}
h_{\pi}\left(\sigma^{k}, \nu \circ \sigma^{-1}\right) & =H_{\nu \circ \sigma^{-1}}\left(\mathcal{P}_{0}^{k-1} \mid \sigma^{-k} \pi^{-1} \gamma\right)-H_{\nu \circ \sigma^{-1}}\left(\mathcal{P}_{0}^{k-1} \mid \pi^{-1} \gamma\right) \\
& =\sum_{j=0}^{k-1}\left(H_{\nu \circ \sigma^{-j-1}}\left(\mathcal{P} \mid \sigma^{-1} \pi^{-1} \gamma\right)-H_{\nu \circ \sigma^{-j-1}}\left(\mathcal{P} \mid \pi^{-1} \gamma\right)\right) .
\end{aligned}
$$

Since $v$ is $\sigma^{k}$-invariant, we obtain $h_{\pi}\left(\sigma^{k}, v \circ \sigma^{-1}\right)=h_{\pi}\left(\sigma^{k}, v\right)$. This finishes the proof of the claim. 
To complete the proof of the proposition, let $m=\frac{1}{k} \sum_{i=0}^{k-1} \nu \circ \sigma^{-i}$. It is clear that $m$ is $\sigma$-invariant. By Proposition 4.1(ii), $h_{\pi}\left(\sigma^{k}, \cdot\right)$ is affine on $\mathcal{M}_{\sigma^{k}}(\Sigma)$. Hence

$$
h_{\pi}\left(\sigma^{k}, m\right)=\frac{1}{k} \sum_{i=0}^{k-1} h_{\pi}\left(\sigma^{k}, v \circ \sigma^{-i}\right)=h_{\pi}\left(\sigma^{k}, v\right) \text {. }
$$

Combining this with Lemma 4.8 yields the equality $h_{\pi}(\sigma, m)=\frac{1}{k} h_{\pi}\left(\sigma^{k}, v\right)$.

\subsection{A Version of the Shannon-McMillan-Breiman Theorem Associated with IFSs}

In this subsection, we prove the following Shannon-McMillan-Breiman type theorem associated with IFSs, which is needed in the proof of Theorem 2.11. It is also of independent interest.

PROPOSITION 4.10 Let $\left\{S_{i}\right\}_{i=1}^{\ell}$ be an IFS and $m \in \mathcal{M}_{\sigma}(\Sigma)$. Then

$$
\lim _{k \rightarrow \infty} \frac{1}{k} \mathbf{I}_{m}\left(\mathcal{P}_{0}^{k-1} \mid \pi^{-1} \gamma\right)(x)=\mathbf{E}_{m}(f \mid \mathcal{I})(x)=h(\sigma, m, x)-h_{\pi}(\sigma, m, x)
$$

almost everywhere and in $L^{1}$, where

$$
f:=\mathbf{I}_{m}\left(\mathcal{P} \mid \sigma^{-1} \mathcal{B}(\Sigma)\right)+\mathbf{I}_{m}\left(\mathcal{P} \mid \pi^{-1} \gamma\right)-\mathbf{I}_{m}\left(\mathcal{P} \mid \sigma^{-1} \pi^{-1} \gamma\right),
$$

$\mathcal{I}=\left\{B \in \mathcal{B}(\Sigma): \sigma^{-1} B=B\right\}$, and $h(\sigma, m, x), h_{\pi}(\sigma, m, x)$ denote the classical local entropy and the local projection entropy of $m$ at $x$ (see Definition 2.1), respectively. Moreover, if $m$ is ergodic, then the limit in (4.10) equals $h(\sigma, m)-h_{\pi}(\sigma, m)$ for $m$-a.e. $x \in \Sigma$.

Remark 4.11. If $\xi$ is a countable Borel partition of $\Sigma$, and $\mathcal{A} \subset \mathcal{B}(\Sigma)$ is a sub- $\sigma$ algebra with $\sigma^{-1} \mathcal{A}=\mathcal{A}$, then the relativized Shannon-McMillan-Breiman theorem states that

$$
\lim _{k \rightarrow \infty} \frac{1}{k} \mathbf{I}_{m}\left(\xi_{0}^{k-1} \mid \mathcal{A}\right)(x)=\mathbf{E}_{m}(g \mid \mathcal{I})(x) \quad \text { for } m \text {-a.e. } x \in \Sigma,
$$

where $g=\mathbf{I}_{m}\left(\xi \mid \mathcal{A} \vee \xi_{1}^{\infty}\right)$ (see, e.g., [8, lemma 4.1]). However, under the setting of Proposition 4.10, the sub- $\sigma$-algebra $\pi^{-1} \gamma$ is not invariant in general.

In the following we present a generalized version of Proposition 4.10.

Proposition 4.12 Let $\xi$ be a countable Borel partition of $\Sigma$ with $H_{m}(\xi)<\infty$, and let $\mathcal{A} \subset \mathcal{B}(\Sigma)$ be a sub- $\sigma$-algebra so that $\hat{\xi} \vee \sigma^{-1} \mathcal{A}=\widehat{\xi} \vee \mathcal{A}$. Let $m \in \mathcal{M}_{\sigma}(\Sigma)$. Then

$$
\lim _{k \rightarrow \infty} \frac{1}{k} \mathbf{I}_{m}\left(\xi_{0}^{k-1} \mid \mathcal{A}\right)(x)=\mathbf{E}_{m}(f \mid \mathcal{I})(x)
$$


almost everywhere and in $L^{1}$, where

$$
f:=\mathbf{I}_{m}\left(\xi \mid \sigma^{-1} \mathcal{A} \vee \bigvee_{i=1}^{\infty} \sigma^{-i} \hat{\xi}\right)+\mathbf{I}_{m}(\xi \mid \mathcal{A})-\mathbf{I}_{m}\left(\xi \mid \sigma^{-1} \mathcal{A}\right)
$$

and $\mathcal{I}=\left\{B \in \mathcal{B}(\Sigma): \sigma^{-1} B=B\right\}$.

To prove Proposition 4.12, we need the following lemma:

LEMMA 4.13 ([42], corollary 1.6, p. 96) Let $m \in \mathcal{M}_{\sigma}(\Sigma)$. Let $F_{k} \in L^{1}(\Sigma, m)$ be a sequence that converges almost everywhere and in $L^{1}$ to $F \in L^{1}(\Sigma, m)$. Then

$$
\lim _{k \rightarrow \infty} \frac{1}{k} \sum_{j=0}^{k-1} F_{k-j}\left(\sigma^{j}(x)\right)=\mathbf{E}_{m}(F \mid \mathcal{I})(x)
$$

almost everywhere and in $L^{1}$.

Proof of Proposition 4.12: For $k \geq 2$ and $x \in \Sigma$, we write

$$
g_{k}(x)=\mathbf{I}_{m}\left(\xi_{0}^{k-1} \mid \mathcal{A}\right)(x)-\mathbf{I}_{m}\left(\xi_{0}^{k-2} \mid \mathcal{A}\right)(\sigma x) .
$$

Then

$$
\mathbf{I}_{m}\left(\xi_{0}^{k-1} \mid \mathcal{A}\right)(x)=\mathbf{I}_{m}(\xi \mid \mathcal{A})\left(\sigma^{k-1} x\right)+\sum_{j=0}^{k-2} g_{k-j}\left(\sigma^{j} x\right) .
$$

We claim that

$$
\begin{aligned}
g_{k}(x)= & \mathbf{I}_{m}\left(\xi \mid \sigma^{-1} \mathcal{A} \vee \bigvee_{i=1}^{k-1} \sigma^{-i} \hat{\xi}\right)(x)+\mathbf{I}_{m}(\xi \mid \mathcal{A})(x) \\
& -\mathbf{I}_{m}\left(\xi \mid \sigma^{-1} \mathcal{A}\right)(x)
\end{aligned}
$$

By the claim and Lemma 4.4(iv), $g_{k}$ converges almost everywhere and in $L^{1}$ to $f$. Hence (4.11) follows from (4.12) and Lemma 4.13.

Now we turn to the proof of (4.13). Let $k \geq 2$. We have

$$
\begin{aligned}
& \mathbf{I}_{m}\left(\xi_{0}^{k-1} \mid \sigma^{-1} \mathcal{A}\right)(x) \\
& \quad=\mathbf{I}_{m}\left(\xi \mid \sigma^{-1} \mathcal{A}\right)(x)+\mathbf{I}_{m}\left(\bigvee_{i=1}^{k-1} \sigma^{-i} \xi \mid \sigma^{-1} \mathcal{A} \vee \hat{\xi}\right)(x) \\
& \quad=\mathbf{I}_{m}\left(\xi \mid \sigma^{-1} \mathcal{A}\right)(x)+\mathbf{I}_{m}\left(\bigvee_{i=1}^{k-1} \sigma^{-i} \xi \mid \mathcal{A} \vee \hat{\xi}\right)(x)
\end{aligned}
$$


using the property $\sigma^{-1} \mathcal{A} \vee \widehat{\xi}=\mathcal{A} \vee \widehat{\xi}$. Meanwhile, we have

$$
\begin{aligned}
\mathbf{I}_{m} & \left(\xi_{0}^{k-1} \mid \sigma^{-1} \mathcal{A}\right)(x) \\
& =\mathbf{I}_{m}\left(\bigvee_{i=1}^{k-1} \sigma^{-i} \xi \mid \sigma^{-1} \mathcal{A}\right)(x)+\mathbf{I}_{m}\left(\xi \mid \sigma^{-1} \mathcal{A} \vee \bigvee_{i=1}^{k-1} \sigma^{-i} \hat{\xi}\right)(x) \\
& =\mathbf{I}_{m}\left(\xi_{0}^{k-2} \mid \mathcal{A}\right)(\sigma x)+\mathbf{I}_{m}\left(\xi \mid \sigma^{-1} \mathcal{A} \vee \bigvee_{i=1}^{k-1} \sigma^{-i} \hat{\xi}\right)(x)
\end{aligned}
$$

Combining (4.14) with (4.15) yields

$$
\begin{aligned}
\mathbf{I}_{m}\left(\xi \mid \sigma^{-1} \mathcal{A}\right)(x)+ & \mathbf{I}_{m}\left(\bigvee_{i=1}^{k-1} \sigma^{-i} \xi \mid \mathcal{A} \vee \hat{\xi}\right)(x)= \\
& \mathbf{I}_{m}\left(\xi_{0}^{k-2} \mid \mathcal{A}\right)(\sigma x)+\mathbf{I}_{m}\left(\xi \mid \sigma^{-1} \mathcal{A} \vee \bigvee_{i=1}^{k-1} \sigma^{-i} \hat{\xi}\right)(x)
\end{aligned}
$$

However,

$$
\mathbf{I}_{m}\left(\xi_{0}^{k-1} \mid \mathcal{A}\right)(x)=\mathbf{I}_{m}(\xi \mid \mathcal{A})(x)+\mathbf{I}_{m}\left(\bigvee_{i=1}^{k-1} \sigma^{-i} \xi \mid \mathcal{A} \vee \hat{\xi}\right)(x)
$$

Combining (4.16) with (4.17) yields (4.13). This finishes the proof of Proposition 4.12 .

We remark that Proposition 4.10 can be stated in terms of conditional measures. To see this, let

$$
\eta=\left\{\pi^{-1}(z): z \in \mathbb{R}^{d}\right\}
$$

be the measurable partition of $\Sigma$ generated by the canonical projection $\pi$ associated with $\left\{S_{i}\right\}_{i=1}^{\ell}$. For $m \in \mathcal{M}_{\sigma}(\Sigma)$, let $\left\{m_{x}^{\eta}\right\}_{x \in \Sigma}$ denote the canonical system of conditional measures with respect to $\eta$. For $x \in \Sigma$ and $k \in \mathbb{N}$, let $\mathcal{P}_{0}^{k}(x)$ denote the element in the partition $\mathcal{P}_{0}^{k}$ containing $x$. Then Proposition 4.10 can be restated as follows:

Proposition 4.14 For $m \in \mathcal{M}_{\sigma}(\Sigma)$, we have

$$
-\lim _{k \rightarrow \infty} \frac{1}{k} \log m_{x}^{\eta}\left(\mathcal{P}_{0}^{k}(x)\right)=\mathbf{E}_{m}(f \mid \mathcal{I})(x) \quad \text { for m-a.e. } x \in \Sigma,
$$

where $f:=\mathbf{I}_{m}\left(\mathcal{P} \mid \sigma^{-1} \mathcal{B}(\Sigma)\right)+\mathbf{I}_{m}\left(\mathcal{P} \mid \pi^{-1} \gamma\right)-\mathbf{I}_{m}\left(\mathcal{P} \mid \sigma^{-1} \pi^{-1} \gamma\right)$. Moreover, if $m$ is ergodic, then the limit in (4.18) equals $h(\sigma, m)-h_{\pi}(\sigma, m)$ for $m$-a.e. $x \in \Sigma$.

PROOF: It suffices to show that for each $k \in \mathbb{N}$,

$$
\log m_{x}^{\eta}\left(\mathcal{P}_{0}^{k}(x)\right)=-\mathbf{I}_{m}\left(\mathcal{P}_{0}^{k} \mid \pi^{-1} \gamma\right)(x) \quad \text { almost everywhere. }
$$


To see this, by Theorem 3.1 we have

$$
\sum_{A \in \mathcal{P}_{0}^{k}} \chi_{A}(x) m_{x}^{\eta}(A)=\sum_{A \in \mathcal{P}_{0}^{k}} \chi_{A}(x) \mathbf{E}_{m}\left(\chi_{A} \mid \pi^{-1} \gamma\right)(x) \quad \text { for } m \text {-a.e. } x \in \Sigma .
$$

Taking the logarithm yields the desired result.

Remark 4.15. In Proposition 4.14, for $m$-a.e. $x \in \Sigma$, we have

$$
\lim _{k \rightarrow \infty}-\frac{1}{k} \log m_{x}^{\eta}\left(\mathcal{P}_{0}^{k}(y)\right)=\mathbf{E}_{m}(f \mid \mathcal{I})(y) \quad \text { for } m_{x}^{\eta} \text {-a.e. } y \in \eta(x) .
$$

To see this, denote

$$
R=\left\{y \in \Sigma:-\lim _{k \rightarrow \infty} \frac{1}{k} \log m_{y}^{\eta}\left(\mathcal{P}_{0}^{k}(y)\right)=\mathbf{E}_{m}(f \mid \mathcal{I})(y)\right\} .
$$

Then $1=m(R)=\int m_{x}^{\eta}(R \cap \eta(x)) d m(x)$. Hence $m_{x}^{\eta}(R \cap \eta(x))=1 m$-a.e. For $y \in R \cap \eta(x)$, we have

$$
\lim _{k \rightarrow \infty}-\frac{1}{k} \log m_{x}^{\eta}\left(\mathcal{P}_{0}^{k}(y)\right)=\lim _{k \rightarrow \infty}-\frac{1}{k} \log m_{y}^{\eta}\left(\mathcal{P}_{0}^{k}(y)\right)=\mathbf{E}_{m}(f \mid \mathcal{I})(y) .
$$

As a corollary of Proposition 4.14, we have the following:

COROLlary 4.16 Let $m \in \mathcal{M}_{\sigma}(\Sigma)$. Then

$$
\begin{aligned}
h_{\pi}(\sigma, m)=h(\sigma, m) & \Longleftrightarrow \lim _{k \rightarrow \infty} \frac{1}{k} \log m_{x}^{\eta}\left(\mathcal{P}_{0}^{k}(x)\right)=0 \text { m-a.e. } \\
& \Longleftrightarrow \operatorname{dim}_{H} m_{x}^{\eta}=0 \text { m-a.e. }
\end{aligned}
$$

In particular, if $\operatorname{dim}_{H} \pi^{-1}(z)=0$ for each $z \in \mathbb{R}^{d}$, then $h_{\pi}(\sigma, m)=h(\sigma, m)$. Here $\operatorname{dim}_{H}$ denotes the Hausdorff dimension.

Proof: Let $f$ be defined as in Proposition 4.14. Then

$$
\int \mathbf{E}_{m}(f \mid \mathcal{I}) d m=\int f d m=h(\sigma, m)-h_{\pi}(\sigma, m) .
$$

By (4.18), $\mathbf{E}_{m}(f \mid \mathcal{I})(x) \geq 0$ for $m$-a.e. $x \in \Sigma$. Hence we have

$$
\begin{aligned}
h(\sigma, m)=h_{\pi}(\sigma, m) & \Longleftrightarrow \mathbf{E}_{m}(f \mid \mathcal{I})=0 m \text {-a.e. } \\
& \Longleftrightarrow \lim _{k \rightarrow \infty} \frac{1}{k} \log m_{x}^{\eta}\left(\mathcal{P}_{0}^{k}(x)\right)=0 m \text {-a.e. }
\end{aligned}
$$

Using dimension theory of measures (see, e.g., [14]), we have

$$
\operatorname{dim}_{H} m_{x}^{\eta}=\operatorname{ess} \sup _{y \in \eta(x)} \liminf _{k \rightarrow \infty} \frac{\log m_{x}^{\eta}\left(\mathcal{P}_{0}^{k}(y)\right)}{\log \ell^{-k}} .
$$

This together with Remark 4.15 yields

$$
\mathbf{E}_{m}(f \mid \mathcal{I})=0 m \text {-a.e. } \Longleftrightarrow \operatorname{dim}_{H} m_{x}^{\eta}=0 m \text {-a.e. }
$$

This finishes the proof of the first part of the corollary. 
To complete the proof, assume that $\operatorname{dim}_{H} \pi^{-1}(z)=0$ for each $z \in \mathbb{R}^{d}$. Then for each $x \in \Sigma, \operatorname{dim}_{H} \eta(x)=0$ and hence $\operatorname{dim}_{H} m_{x}^{\eta}=0$. Thus $h_{\pi}(\sigma, m)=$ $h(\sigma, m)$.

\subsection{Projection Entropy under the Ergodic Decomposition}

In this subsection, we first prove the following result:

Proposition 4.17 Let $\left\{S_{i}\right\}_{i=1}^{\ell}$ be an IFS and $m \in \mathcal{M}_{\sigma}(\Sigma)$. Assume that $m=$ $\int \nu d \mathbb{P}(v)$ is the ergodic decomposition of $m$. Then

$$
h_{\pi}(\sigma, m)=\int h_{\pi}(\sigma, v) d \mathbb{P}(v) .
$$

Proof: Let $\mathcal{I}$ denote the $\sigma$-algebra $\left\{B \in \mathcal{B}(\Sigma): \sigma^{-1} B=B\right\}$, and let $m \in \mathcal{M}_{\sigma}(\Sigma)$. Then there exists an $m$-measurable partition $\varepsilon$ of $\Sigma$ such that $\widehat{\varepsilon}=\mathcal{I}$ modulo sets of zero $m$-measure (see [47, pp. 37-38]). Let $\left\{m_{x}^{\varepsilon}\right\}$ denote the conditional measures of $m$ associated with the partition $\varepsilon$. Then $m=\int m_{x}^{\varepsilon} d m(x)$ is just the ergodic decomposition of $m$ (see, e.g., [31, theorem 2.3.3]). Hence to prove the proposition, we need to show that

$$
h_{\pi}(\sigma, m)=\int h_{\pi}\left(\sigma, m_{x}^{\varepsilon}\right) d m(x) .
$$

We first show the direction " $\leq$ " in (4.19). Note that $\mathcal{I}$ is $\sigma$-invariant and $\widehat{\mathcal{P}} \vee$ $\sigma^{-1} \pi^{-1} \gamma=\hat{\mathcal{P}} \vee \pi^{-1} \gamma$. Hence we have $\hat{\mathcal{P}} \vee \sigma^{-1} \pi^{-1} \gamma \vee \mathcal{I}=\hat{\mathcal{P}} \vee \pi^{-1} \gamma \vee \mathcal{I}$. Taking $\xi=\mathcal{P}$ and $\mathcal{A}=\pi^{-1} \gamma \vee \mathcal{I}$ in Proposition 4.12 yields

$$
\lim _{k \rightarrow \infty} \frac{1}{k} \mathbf{I}_{m}\left(\mathcal{P}_{0}^{k-1} \mid \pi^{-1} \gamma \vee \mathcal{I}\right)(x)=\mathbf{E}_{m}(f \mid \mathcal{I})(x)
$$

almost everywhere and in $L^{1}$, where

$$
f:=\mathbf{I}_{m}\left(\mathcal{P} \mid \sigma^{-1} \mathcal{B}(\Sigma)\right)+\mathbf{I}_{m}\left(\mathcal{P} \mid \pi^{-1} \gamma \vee \mathcal{I}\right)-\mathbf{I}_{m}\left(\mathcal{P} \mid \sigma^{-1} \pi^{-1} \gamma \vee \mathcal{I}\right)
$$

By Remark 3.4(ii), we have

$$
\mathbf{I}_{m_{x}^{\varepsilon}}\left(\mathcal{P}_{0}^{k-1} \mid \pi^{-1} \gamma\right)(x)=\mathbf{I}_{m}\left(\mathcal{P}_{0}^{k-1} \mid \pi^{-1} \gamma \vee \mathcal{I}\right)(x)
$$

Hence according to the ergodicity of $m_{x}^{\varepsilon}$ and Proposition 4.10, we have

$$
\begin{aligned}
h\left(\sigma, m_{x}^{\varepsilon}\right)-h_{\pi}\left(\sigma, m_{x}^{\varepsilon}\right) & =\lim _{k \rightarrow \infty} \frac{1}{k} \mathbf{I}_{m_{x}^{\varepsilon}}\left(\mathcal{P}_{0}^{k-1} \mid \pi^{-1} \gamma\right)(x) \\
& =\lim _{k \rightarrow \infty} \frac{1}{k} \mathbf{I}_{m}\left(\mathcal{P}_{0}^{k-1} \mid \pi^{-1} \gamma \vee \mathcal{I}\right)(x)
\end{aligned}
$$

almost everywhere and

$$
\int h\left(\sigma, m_{x}^{\varepsilon}\right)-h_{\pi}\left(\sigma, m_{x}^{\varepsilon}\right) d m(x)=\lim _{k \rightarrow \infty} \frac{1}{k} H_{m}\left(\mathcal{P}_{0}^{k-1} \mid \pi^{-1} \gamma \vee \mathcal{I}\right)
$$

Using Proposition 4.10 again we have

$$
h(\sigma, m)-h_{\pi}(\sigma, m)=\lim _{k \rightarrow \infty} \frac{1}{k} H_{m}\left(\mathcal{P}_{0}^{k-1} \mid \pi^{-1} \gamma\right) .
$$


However, $H_{m}\left(\mathcal{P}_{0}^{k-1} \mid \pi^{-1} \gamma \vee \mathcal{I}\right) \leq H_{m}\left(\mathcal{P}_{0}^{k-1} \mid \pi^{-1} \gamma\right)$ (see, e.g., theorem 4.3(v) in [63]). By (4.21), (4.22), and the above inequality, we have

$$
\int h\left(\sigma, m_{x}^{\varepsilon}\right)-h_{\pi}\left(\sigma, m_{x}^{\varepsilon}\right) d m(x) \leq h(\sigma, m)-h_{\pi}(\sigma, m) .
$$

It is well-known (see [63, theorem 8.4]) that $\int h\left(\sigma, m_{x}^{\varepsilon}\right) d m(x)=h(\sigma, m)$. Hence we obtain the inequality $h_{\pi}(\sigma, m) \leq \int h_{\pi}\left(\sigma, m_{x}^{\varepsilon}\right) d m(x)$.

Now we prove the direction " $\geq$ " in (4.19). For any $n \in \mathbb{N}$, let $\mathcal{D}_{n}$ be defined as in (4.5). Since $\widehat{\mathcal{D}_{n}} \uparrow \gamma$, we have

$$
h_{\pi}(\sigma, m)=\lim _{n \rightarrow \infty} H_{m}\left(\mathcal{P} \mid \sigma^{-1} \pi^{-1} \hat{\mathcal{D}}_{n}\right)-H_{m}\left(\mathcal{P} \mid \pi^{-1} \widehat{\mathcal{D}_{n}}\right)
$$

Now fix $n \in \mathbb{N}$ and denote $A(m)=H_{m}\left(\mathcal{P} \mid \sigma^{-1} \pi^{-1} \widehat{\mathcal{D}_{n}}\right)-H_{m}\left(\mathcal{P} \mid \pi^{-1} \hat{\mathcal{D}_{n}}\right)$ and

$$
\begin{aligned}
B(m) & =H_{m}\left(\sigma^{-1} \pi^{-1} \mathcal{D}_{n} \mid \mathcal{P} \vee \pi^{-1} \hat{\mathcal{D}_{n}}\right) \\
& =H_{m}\left(\mathcal{P} \vee \sigma^{-1} \pi^{-1} \mathcal{D}_{n} \vee \pi^{-1} \mathcal{D}_{n}\right)-H_{m}\left(\mathcal{P} \vee \pi^{-1} \mathcal{D}_{n}\right)
\end{aligned}
$$

Then by (4.6) and (4.7), we have

$$
B(m)-c \leq A(m) \leq B(m),
$$

where $c=d \log (\sqrt{d}+1)$. As a conditional entropy function, $B(m)$ is concave on $\mathcal{M}_{\sigma}(\Sigma)$ (see, e.g., [25, lemma 3.3(1)]). Hence by Jensen's inequality and (4.24), we have

$$
A(m) \geq B(m)-c \geq \int B\left(m_{x}^{\varepsilon}\right) d m(x)-c \geq \int A\left(m_{x}^{\varepsilon}\right) d m(x)-c .
$$

That is,

$$
\begin{aligned}
H_{m}\left(\mathcal{P} \mid \sigma^{-1} \pi^{-1} \hat{\mathcal{D}_{n}}\right)-H_{m}\left(\mathcal{P} \mid \pi^{-1} \hat{\mathcal{D}_{n}}\right) \geq \\
\qquad H_{m_{x}^{\varepsilon}}\left(\mathcal{P} \mid \sigma^{-1} \pi^{-1} \hat{\mathcal{D}_{n}}\right)-H_{m_{x}^{\varepsilon}}\left(\mathcal{P} \mid \pi^{-1} \hat{\mathcal{D}_{n}}\right) d m(x)-c .
\end{aligned}
$$

Letting $n \rightarrow \infty$ and using (4.23) and the Lebesgue dominated convergence theorem, we have

$$
h_{\pi}(\sigma, m) \geq \int h_{\pi}\left(\sigma, m_{x}^{\varepsilon}\right) d m(x)-c .
$$

Replacing $\sigma$ by $\sigma^{k}$ we have

$$
h_{\pi}\left(\sigma^{k}, m\right) \geq \int h_{\pi}\left(\sigma^{k}, m_{x}^{\varepsilon_{k}}\right) d m(x)-c,
$$

where $\varepsilon_{k}$ denotes a measurable partition of $\Sigma$ such that

$$
\widehat{\varepsilon_{k}}=\left\{B \in \mathcal{B}(\Sigma): \sigma^{-k} B=B\right\}
$$

modulo sets of zero $m$-measure. Note that $m=\int m_{x}^{\varepsilon_{k}} d m(x)$ is the ergodic decomposition of $m$ with respect to $\sigma^{k}$. Hence $m=\int \frac{1}{k} \sum_{i=0}^{k-1} m_{x}^{\varepsilon_{k}} \circ \sigma^{-i} d m(x)$ is 
the ergodic decomposition of $m$ with respect to $\sigma$. It follows that

$$
\frac{1}{k} \sum_{i=0}^{k-1} m_{x}^{\varepsilon_{k}} \circ \sigma^{-i}=m_{x}^{\varepsilon} \quad m \text {-a.e. }
$$

By (4.25), Proposition 4.3, and (4.26), we have

$$
\begin{aligned}
h_{\pi}\left(\sigma^{k}, m\right) & =\frac{1}{k} \sum_{i=0}^{k-1} h_{\pi}\left(\sigma^{k}, m \circ \sigma^{-i}\right) \\
& \geq \frac{1}{k} \sum_{i=0}^{k-1} \int h_{\pi}\left(\sigma^{k}, m_{x}^{\varepsilon_{k}} \circ \sigma^{-i}\right) d m(x)-c \\
& =\int h_{\pi}\left(\sigma^{k}, \frac{1}{k} \sum_{i=0}^{k-1} m_{x}^{\varepsilon_{k}} \circ \sigma^{-i}\right) d m(x)-c \\
& =\int h_{\pi}\left(\sigma^{k}, m_{x}^{\varepsilon}\right) d m(x)-c .
\end{aligned}
$$

Using Proposition 4.3 again yields

$$
h_{\pi}(\sigma, m) \geq \int h_{\pi}\left(\sigma, m_{x}^{\varepsilon}\right) d m(x)-\frac{c}{k} \quad \text { for any } k \in \mathbb{N} .
$$

Hence we have $h_{\pi}(\sigma, m) \geq \int h_{\pi}\left(\sigma, m_{x}^{\varepsilon}\right) d m(x)$, as desired.

Proof of Theorem 2.2: It follows directly from Propositions 4.1, 4.10, and 4.17 .

\subsection{Projection Entropy for Certain Affine IFSs and Proof of Theorem 2.3}

In this subsection, we assume that $\Phi=\left\{S_{i}\right\}_{i=1}^{\ell}$ is an IFS on $\mathbb{R}^{d}$ of the form

$$
S_{i}(x)=A x+c_{i}, \quad i=1, \ldots, \ell,
$$

where $A$ is a $d \times d$ nonsingular real matrix with $\|A\|<1$ and $c_{i} \in \mathbb{R}^{d}$. Let $K$ denote the attractor of $\Phi$.

Let $\mathcal{Q}$ denote the partition $\left\{[0,1)^{d}+\alpha: \alpha \in \mathbb{Z}^{d}\right\}$ of $\mathbb{R}^{d}$. For $n=0,1, \ldots$, and $x \in \mathbb{R}^{d}$, we set

$$
\mathcal{Q}_{n}=\left\{A^{n} Q: Q \in \mathcal{Q}\right\}, \quad \mathcal{Q}_{n}+x=\left\{A^{n} Q+x: Q \in \mathcal{Q}\right\} .
$$

We have the following geometric characterization of $h_{\pi}$ for the IFS $\Phi$ (i.e., Theorem 2.3).

PROPOSITION 4.18

(i) Let $m \in \mathcal{M}_{\sigma}(\Sigma)$. Then

$$
h_{\pi}(\sigma, m)=\lim _{n \rightarrow \infty} \frac{H_{m}\left(\pi^{-1} \mathcal{Q}_{n}\right)}{n} .
$$


(ii)

$$
\lim _{n \rightarrow \infty} \frac{\log \#\left\{Q \in \mathcal{Q}: A^{n} Q \cap K \neq \varnothing\right\}}{n}=\sup \left\{h_{\pi}(\sigma, m): m \in \mathcal{M}_{\sigma}(\Sigma)\right\} .
$$

To prove the above proposition, we need the following lemma:

LEMMA 4.19 Assume that $\Omega$ is a subset of $\{1, \ldots, \ell\}$ such that $S_{i}(K) \cap S_{j}(K)=$ $\varnothing$ for all $i, j \in \Omega$ with $i \neq j$. Suppose that $v$ is an invariant measure on $\Sigma$ supported on $\Omega^{\mathbb{N}}$, i.e., $v([j])=0$ for all $j \in\{1, \ldots, \ell\} \backslash \Omega$. Then $h_{\pi}(\sigma, v)=$ $h(\sigma, v)$.

PROOF: It suffices to prove that $h_{\pi}(\sigma, v) \geq h(\sigma, v)$. Recall that

$$
h_{\pi}(\sigma, v)=H_{\nu}\left(\mathcal{P} \mid \sigma^{-1} \pi^{-1} \gamma\right)-H_{\nu}\left(\mathcal{P} \mid \pi^{-1} \gamma\right)
$$

and $H_{\nu}\left(\mathcal{P} \mid \sigma^{-1} \pi^{-1} \gamma\right) \geq H_{\nu}\left(\mathcal{P} \mid \sigma^{-1} \mathcal{B}(\Sigma)\right)=h(\sigma, v)$. Hence we only need to show $H_{\nu}\left(\mathcal{P} \mid \pi^{-1} \gamma\right)=0$. To do this, denote

$$
\delta=\min \left\{d\left(S_{i}(K), S_{j}(K)\right): i, j \in \Omega, i \neq j\right\} .
$$

Then $\delta>0$. Let $\xi$ be an arbitrary finite Borel partition of $K$ so that $\operatorname{diam}(A)<\frac{\delta}{2}$ for $A \in \xi$. Set $\mathcal{W}=\{[i]: i \in \Omega\}$. Since $\nu$ is supported on $\Omega^{\mathbb{N}}$, we have

$$
\begin{aligned}
H_{\nu}\left(\mathcal{P} \mid \pi^{-1} \hat{\xi}\right) & =H_{\nu}\left(\mathcal{P} \vee \pi^{-1} \xi\right)-H_{v}\left(\pi^{-1} \xi\right) \\
& =H_{\nu}\left(\mathcal{W} \vee \pi^{-1} \xi\right)-H_{\nu}\left(\pi^{-1} \xi\right)
\end{aligned}
$$

However, for each $A \in \xi$, there is at most one $i \in \Omega$ such that $S_{i}(K) \cap A \neq \varnothing$, i.e., $[i] \cap \pi^{-1} A \neq \varnothing$. This forces $H_{v}\left(\mathcal{W} \vee \pi^{-1} \xi\right)=H_{v}\left(\pi^{-1} \xi\right)$. Hence

$$
H_{\nu}\left(\mathcal{P} \mid \pi^{-1} \widehat{\xi}\right)=0
$$

By the arbitrariness of $\xi$ and Lemma 4.4(iv), we have $H_{v}\left(\mathcal{P} \mid \pi^{-1} \gamma\right)=0$.

Proof of Proposition 4.18: We first prove (i). Let $m \in \mathcal{M}_{\sigma}(\Sigma)$. Denote $\gamma=\mathcal{B}\left(\mathbb{R}^{d}\right)$. According to Proposition 4.3, we have

$$
H_{m}\left(\mathcal{P}_{0}^{p-1} \mid \sigma^{-p} \pi^{-1} \gamma\right)-H_{m}\left(\mathcal{P}_{0}^{p-1} \mid \pi^{-1} \gamma\right)=p h_{\pi}(\sigma, m), \quad p \in \mathbb{N} .
$$

Now fix $p$. Since $\hat{\mathcal{Q}_{n}} \uparrow \gamma$, by Lemma 4.4(iv), there exists $k_{0}$ such that for $k \geq k_{0}$,

$$
\left|H_{m}\left(\mathcal{P}_{0}^{p-1} \mid \sigma^{-p} \pi^{-1} \gamma\right)-H_{m}\left(\mathcal{P}_{0}^{p-1} \mid \sigma^{-p} \pi^{-1} \widehat{\mathcal{Q}_{k p}}\right)\right| \leq 1
$$

and

$$
\left|H_{m}\left(\mathcal{P}_{0}^{p-1} \mid \pi^{-1} \gamma\right)-H_{m}\left(\mathcal{P}_{0}^{p-1} \mid \pi^{-1} \mathcal{Q}_{(k+1) p}\right)\right| \leq 1 .
$$

It follows that for $k \geq k_{0}$,

$$
\begin{aligned}
p h_{\pi}(\sigma, m)-2 & \leq H_{m}\left(\mathcal{P}_{0}^{p-1} \mid \sigma^{-p} \pi^{-1} \hat{\mathcal{Q}_{k p}}\right)-H_{m}\left(\mathcal{P}_{0}^{p-1} \mid \pi^{-1} \mathcal{Q}_{(k+1) p}\right) \\
& \leq p h_{\pi}(\sigma, m)+2
\end{aligned}
$$


Now we estimate the difference of conditional entropies in (4.28). Note that

$$
\begin{aligned}
H_{m}\left(\mathcal{P}_{0}^{p-1} \mid \sigma^{-p} \pi^{-1} \hat{\mathcal{Q}_{k p}}\right) & =H_{m}\left(\mathcal{P}_{0}^{p-1} \vee \sigma^{-p} \pi^{-1} \mathcal{Q}_{k p}\right)-H_{m}\left(\sigma^{-p} \pi^{-1} \mathcal{Q}_{k p}\right) \\
& =H_{m}\left(\mathcal{P}_{0}^{p-1} \vee \sigma^{-p} \pi^{-1} \mathcal{Q}_{k p}\right)-H_{m}\left(\pi^{-1} \mathcal{Q}_{k p}\right)
\end{aligned}
$$

and

$$
H_{m}\left(\mathcal{P}_{0}^{p-1} \mid \pi^{-1} \mathcal{Q}_{(\hat{k+1) p}}\right)=H_{m}\left(\mathcal{P}_{0}^{p-1} \vee \pi^{-1} \mathcal{Q}_{(k+1) p}\right)-H_{m}\left(\pi^{-1} \mathcal{Q}_{(k+1) p}\right) .
$$

Hence we have

$$
\begin{aligned}
H_{m}\left(\mathcal{P}_{0}^{p-1} \mid \sigma^{-p} \pi^{-1} \hat{\mathcal{Q}_{k p}}\right)-H_{m}\left(\mathcal{P}_{0}^{p-1} \mid \pi^{-1} \hat{\mathcal{Q}_{(k+1) p}}\right) \\
=H_{m}\left(\mathcal{P}_{0}^{p-1} \vee \sigma^{-p_{\pi}} \overline{\mathcal{Q}}_{k p}\right)-H_{m}\left(\mathcal{P}_{0}^{p-1} \vee \pi^{-1} \mathcal{Q}_{(k+1) p}\right) \\
\quad+H_{m}\left(\pi^{-1} \mathcal{Q}_{(k+1) p}\right)-H_{m}\left(\pi^{-1} \mathcal{Q}_{k p}\right)
\end{aligned}
$$

Observe that for each $[u] \in \mathcal{P}_{0}^{p-1}$ and any $Q \in \mathcal{Q}$,

$$
[u] \cap \sigma^{-p} \pi^{-1} A^{k p} Q=[u] \cap \pi^{-1} S_{u} A^{k p} Q .
$$

Since the linear part of $S_{u}$ is $A^{p}$, the set $S_{u} A^{k p} Q$ intersects at most $2^{d}$ elements of $\mathcal{Q}_{(k+1) p}$. Therefore each element of $\mathcal{P}_{0}^{p-1} \vee \sigma^{-p} \pi^{-1} \mathcal{Q}_{k p}$ intersects at most $2^{d}$ elements of $\mathcal{P}_{0}^{p-1} \vee \pi^{-1} \mathcal{Q}_{(k+1) p}$. Similarly, the statement is also true if the two partitions are interchanged. Therefore by Lemma 4.6 we have

$$
\left|H_{m}\left(\mathcal{P}_{0}^{p-1} \vee \sigma^{-p} \pi^{-1} \mathcal{Q}_{k p}\right)-H_{m}\left(\mathcal{P}_{0}^{p-1} \vee \pi^{-1} \mathcal{Q}_{(k+1) p}\right)\right| \leq d \log 2 .
$$

This together with (4.28) and (4.29) yields

$$
\begin{aligned}
p h_{\pi}(\sigma, m)-2-d \log 2 & \leq H_{m}\left(\pi^{-1} \mathcal{Q}_{(k+1) p}\right)-H_{m}\left(\pi^{-1} \mathcal{Q}_{k p}\right) \\
& \leq p h_{\pi}(\sigma, m)+2+d \log 2
\end{aligned}
$$

for $k \geq k_{0}$. Hence we have

$$
\limsup _{k \rightarrow \infty} \frac{H_{m}\left(\pi^{-1} \mathcal{Q}_{k p}\right)}{k p} \leq h_{\pi}(\sigma, m)+\frac{2+d \log 2}{p}
$$

and

$$
\liminf _{k \rightarrow \infty} \frac{H_{m}\left(\pi^{-1} \mathcal{Q}_{k p}\right)}{k p} \geq h_{\pi}(\sigma, m)-\frac{2+d \log 2}{p} .
$$

By a volume argument, there is a large integer $N$ ( $N$ depends on $A, d$, and $p$ and is independent of $k$ ) such that for any $i=0,1, \ldots, p-1$, each element of $\mathcal{Q}_{k p+i}$ intersects at most $N$ elements of $\mathcal{Q}_{k p}$, and vice versa. Hence by Lemma 4.6, $\left|H_{m}\left(\pi^{-1} \mathcal{Q}_{k p}\right)-H_{m}\left(\pi^{-1} \mathcal{Q}_{k p+i}\right)\right|<\log N$ for $0 \leq i \leq p-1$. It follows that

$$
\limsup _{k \rightarrow \infty} \frac{H_{m}\left(\pi^{-1} \mathcal{Q}_{k p}\right)}{k p}=\limsup _{n \rightarrow \infty} \frac{H_{m}\left(\pi^{-1} \mathcal{Q}_{n}\right)}{n}
$$


and

$$
\liminf _{k \rightarrow \infty} \frac{H_{m}\left(\pi^{-1} \mathcal{Q}_{k p}\right)}{k p}=\liminf _{n \rightarrow \infty} \frac{H_{m}\left(\pi^{-1} \mathcal{Q}_{n}\right)}{n}
$$

Thus we have

$$
\begin{aligned}
h_{\pi}(\sigma, m)-\frac{2+d \log 2}{p} & \leq \liminf _{n \rightarrow \infty} \frac{H_{m}\left(\pi^{-1} \mathcal{Q}_{n}\right)}{n} \\
& \leq \limsup _{n \rightarrow \infty} \frac{H_{m}\left(\pi^{-1} \mathcal{Q}_{n}\right)}{n} \leq h_{\pi}(\sigma, m)+\frac{2+d \log 2}{p} .
\end{aligned}
$$

Letting $p$ tend to infinity, we obtain (4.27).

To show (ii), we assume $K \subset[0,1)^{d}$ without loss of generality. Note that the number of (nonempty) elements in the partition $\pi^{-1} \mathcal{Q}_{n}$ is just equal to

$$
N_{n}:=\#\left\{Q \in \mathcal{Q}: A^{n} Q \cap K \neq \varnothing\right\} .
$$

Hence by (4.2), we have

$$
H_{m}\left(\pi^{-1} \mathcal{Q}_{n}\right) \leq \log N_{n} \quad \forall m \in \mathcal{M}_{\sigma}(\Sigma)
$$

This together with (i) proves

$$
\liminf _{n \rightarrow \infty} \frac{\log N_{n}}{n} \geq \sup \left\{h_{\pi}(\sigma, m): m \in \mathcal{M}_{\sigma}(\Sigma)\right\} .
$$

To prove (ii), we still need to show

$$
\limsup _{n \rightarrow \infty} \frac{\log N_{n}}{n} \leq \sup \left\{h_{\pi}(\sigma, m): m \in \mathcal{M}_{\sigma}(\Sigma)\right\} .
$$

We may assume that $\lim \sup _{n \rightarrow \infty} \log N_{n} / n>0$; otherwise there is nothing to prove. Let $n$ be a large integer so that $N_{n}>7^{d}$. Choose a subset $\Gamma$ of

$$
\left\{Q: A^{n} Q \cap K \neq \varnothing, Q \in \mathcal{Q}\right\}
$$

such that \#Г> $7^{-d} N_{n}$, and

$$
2 Q \cap 2 \widetilde{Q}=\varnothing \quad \text { for different } Q, \widetilde{Q} \in \Gamma,
$$

where $2 Q:=\bigcup_{P \in \mathcal{Q}: \bar{P} \cap \bar{Q} \neq \varnothing} P$, and $\bar{P}$ denotes the closure of $P$. For each $Q \in \Gamma$, since $A^{n} Q \cap K \neq \varnothing$, we can pick a word $u=u(Q) \in \Sigma_{n}$ in such a way that $S_{u} K \cap A^{n} Q \neq \varnothing$.

Consider the collection $W=\{u(Q): Q \in \Gamma\}$. The separation condition (4.31) for elements in $\Gamma$ guarantees that

$$
S_{u(Q)}(K) \cap S_{u(\widetilde{Q})}(K)=\varnothing \quad \text { for all } Q, \widetilde{Q} \in \Gamma \text { with } Q \neq \widetilde{Q} .
$$

Define a Bernoulli measure $v$ on $W^{\mathbb{N}}$ by

$$
v\left(\left[w_{1} \cdots w_{k}\right]\right)=(\# \Gamma)^{-k}, \quad k \in \mathbb{N}, w_{1}, \ldots, w_{k} \in W .
$$


Then $v$ can be viewed as a $\sigma^{n}$-invariant measure on $\Sigma$ (by viewing $W^{\mathbb{N}}$ as a subset of $\Sigma)$. By Lemma 4.19, we have $h_{\pi}\left(\sigma^{n}, v\right)=h\left(\sigma^{n}, v\right)=\log \# \Gamma$. Define $\mu=$ $\frac{1}{n} \sum_{i=0}^{n-1} v \circ \sigma^{-i}$. Then $\mu \in \mathcal{M}_{\sigma}(\Sigma)$, and by Proposition 4.3,

$$
h_{\pi}(\sigma, \mu)=\frac{h_{\pi}\left(\sigma^{n}, v\right)}{n}=\frac{\log \# \Gamma}{n} \geq \frac{\log \left(7^{-d} N_{n}\right)}{n},
$$

from which (4.30) follows.

\subsection{Upper Semicontinuity of $h_{\pi}(\sigma, \cdot)$ under the AWSC}

In this subsection, we prove the following proposition:

Proposition 4.20 Assume that $\left\{S_{i}\right\}_{i=1}^{\ell}$ is an IFS that satisfies the AWSC (see Definition 2.14). Then the map $m \mapsto h_{\pi}(\sigma, m)$ on $\mathcal{M}_{\sigma}(\Sigma)$ is upper semicontinuous.

We first prove a lemma.

LEMMA 4.21 Let $\left\{S_{i}\right\}_{i=1}^{\ell}$ be an IFS with attractor $K \subset \mathbb{R}^{d}$. Assume that

$$
\#\left\{1 \leq i \leq \ell: x \in S_{i}(K)\right\} \leq k
$$

for some $k \in \mathbb{N}$ and each $x \in \mathbb{R}^{d}$. Then $H_{v}\left(\mathcal{P} \mid \pi^{-1} \gamma\right) \leq \log k$ for any Borel probability measure $v$ on $\Sigma$.

PROOF: A compactness argument shows that there is $r_{0}>0$ such that

$$
\#\left\{1 \leq i \leq \ell: B\left(x, r_{0}\right) \cap S_{i}(K) \neq \varnothing\right\} \leq k
$$

for each $x \in \mathbb{R}^{d}$. Let $n \in \mathbb{N}$ so that $2^{-n} \sqrt{d}<r_{0}$. Then for each $Q \in \mathcal{D}_{n}$, where $\mathcal{D}_{n}$ is defined as in (4.5), there are at most $k$ different $i \in\{1, \ldots, \ell\}$ such that $S_{i}(K) \cap Q \neq \varnothing$. It follows that each member in $\pi^{-1} \mathcal{D}_{n}$ intersects at most $k$ members of $\mathcal{P} \vee \pi^{-1} \mathcal{D}_{n}$. By Lemma 4.6, we have

$$
H_{v}\left(\mathcal{P} \mid \pi^{-1} \widehat{\mathcal{D}_{n}}\right)=H_{v}\left(\mathcal{P} \vee \pi^{-1} \mathcal{D}_{n}\right)-H_{v}\left(\pi^{-1} \mathcal{D}_{n}\right) \leq \log k .
$$

Note that $\pi^{-1} \hat{\mathcal{D}_{n}} \uparrow \pi^{-1} \gamma$. Applying Lemma 4.4(iv), we obtain

$$
H_{v}\left(\mathcal{P} \mid \pi^{-1} \gamma\right)=\lim _{n \rightarrow \infty} H_{v}\left(\mathcal{P} \mid \pi^{-1} \hat{\mathcal{D}}_{n}\right) \leq \log k
$$

As a corollary, we have the following:

COROLLARY 4.22 Under the condition of Lemma 4.21, we have

$$
h_{\pi}(\sigma, m) \geq h(\sigma, m)-\log k \quad \text { for any } m \in \mathcal{M}_{\sigma}(\Sigma) .
$$

PROOF: By the definition of $h_{\pi}(\sigma, m)$ and Lemma 4.21, we have

$$
h_{\pi}(\sigma, m)=H_{m}\left(\mathcal{P} \mid \sigma^{-1} \pi^{-1} \gamma\right)-H_{m}\left(\mathcal{P} \mid \pi^{-1} \gamma\right) \geq H_{m}\left(\mathcal{P} \mid \sigma^{-1} \pi^{-1} \gamma\right)-\log k .
$$

However, $H_{m}\left(\mathcal{P} \mid \sigma^{-1} \pi^{-1} \gamma\right) \geq H_{m}\left(\mathcal{P} \mid \sigma^{-1} \mathcal{B}(\Sigma)\right)=h(\sigma, m)$. This implies the desired result. 
To prove Proposition 4.20, we need the following lemma:

LEMMA 4.23 Let $\left\{S_{i}\right\}_{i=1}^{\ell}$ be an IFS with attractor $K$. Suppose that $\Omega$ is a subset of $\{1, \ldots, \ell\}$ such that there is a map $g:\{1, \ldots, \ell\} \rightarrow \Omega$ so that

$$
S_{i}=S_{g(i)}, \quad i=1, \ldots, \ell .
$$

Let $\left(\Omega^{\mathbb{N}}, \tilde{\sigma}\right)$ denote the one-sided full shift over $\Omega$. Define $G: \Sigma \rightarrow \Omega^{\mathbb{N}}$ by $\left(x_{j}\right)_{j=1}^{\infty} \mapsto\left(g\left(x_{j}\right)\right)_{j=1}^{\infty}$. Then

(i) $K$ is also the attractor of $\left\{S_{i}\right\}_{i \in \Omega}$. Moreover, if we let $\tilde{\pi}: \Omega^{\mathbb{N}} \rightarrow K$ denote the canonical projection with respect to $\left\{S_{i}\right\}_{i \in \Omega}$, then we have $\pi=\tilde{\pi} \circ G$.

(ii) Let $m \in \mathcal{M}_{\sigma}(\Sigma)$. Then $v=m \circ G^{-1} \in \mathcal{M}_{\tilde{\sigma}}\left(\Omega^{\mathbb{N}}\right)$. Furthermore, $h_{\pi}(\sigma, m)=h_{\tilde{\pi}}(\widetilde{\sigma}, v)$. In particular, $h_{\pi}(\sigma, m) \leq \log (\# \Omega)$.

Proof: (i) is obvious.

To see (ii), let $m \in \mathcal{M}_{\sigma}(\Sigma)$. It is easily seen that the following diagram commutes:

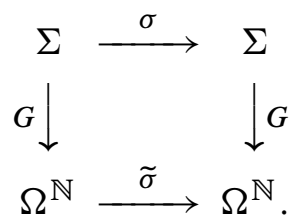

That is, $\widetilde{\sigma} \circ G=G \circ \sigma$. Hence $v=m \circ G^{-1} \in \mathcal{M}_{\tilde{\sigma}}\left(\Omega^{\mathbb{N}}\right)$. To show that $h_{\pi}(\sigma, m)=h_{\tilde{\pi}}(\widetilde{\sigma}, \nu)$, let $\mathcal{Q}=\{[i]: i \in \Omega\}$ be the canonical partition of $\Omega^{\mathbb{N}}$. Then

$$
\begin{aligned}
h_{\tilde{\pi}}(\tilde{\sigma}, v) & =H_{m \circ G^{-1}}\left(\mathcal{Q} \mid \tilde{\sigma}^{-1} \tilde{\pi}^{-1} \gamma\right)-H_{m \circ G^{-1}}\left(\mathcal{Q} \mid \tilde{\pi}^{-1} \gamma\right) \\
& =H_{m}\left(G^{-1}(\mathcal{Q}) \mid G^{-1} \tilde{\sigma}^{-1} \tilde{\pi}^{-1} \gamma\right)-H_{m}\left(G^{-1}(\mathcal{Q}) \mid G^{-1} \tilde{\pi}^{-1} \gamma\right) \\
& =H_{m}\left(G^{-1}(\mathcal{Q}) \mid \sigma^{-1} \pi^{-1} \gamma\right)-H_{m}\left(G^{-1}(\mathcal{Q}) \mid \pi^{-1} \gamma\right),
\end{aligned}
$$

using the facts $G \circ \sigma=\tilde{\sigma} \circ G$ and $\tilde{\pi} \circ G=\pi$. Since $\mathcal{P} \vee G^{-1}(\mathcal{Q})=\mathcal{P}$, we have

$$
\begin{aligned}
h_{\pi}( & \sigma, m)-h_{\tilde{\pi}}\left(\widetilde{\sigma}, m \circ G^{-1}\right) \\
= & \left(H_{m}\left(\mathcal{P} \mid \sigma^{-1} \pi^{-1} \gamma\right)-H_{m}\left(\mathcal{P} \mid \pi^{-1} \gamma\right)\right) \\
& -\left(H_{m}\left(G^{-1}(\mathcal{Q}) \mid \sigma^{-1} \pi^{-1} \gamma\right)-H_{m}\left(G^{-1}(\mathcal{Q}) \mid \pi^{-1} \gamma\right)\right) \\
= & \left(H_{m}\left(\mathcal{P} \mid \sigma^{-1} \pi^{-1} \gamma\right)-H_{m}\left(G^{-1}(\mathcal{Q}) \mid \sigma^{-1} \pi^{-1} \gamma\right)\right) \\
& -\left(H_{m}\left(\mathcal{P} \mid \pi^{-1} \gamma\right)-H_{m}\left(G^{-1}(\mathcal{Q}) \mid \pi^{-1} \gamma\right)\right) \\
= & H_{m}\left(\mathcal{P} \mid \sigma^{-1} \pi^{-1} \gamma \vee G^{-1}(\widehat{\mathcal{Q}})\right)-H_{m}\left(\mathcal{P} \mid \pi^{-1} \gamma \vee G^{-1}(\widehat{\mathcal{Q}})\right) .
\end{aligned}
$$

An argument similar to the proof of Lemma 4.7 shows that

$$
\sigma^{-1} \pi^{-1} \gamma \vee G^{-1}(\widehat{\mathcal{Q}})=\pi^{-1} \gamma \vee G^{-1}(\widehat{\mathcal{Q}}) \text {. }
$$

Hence we have $h_{\pi}(\sigma, m)=h_{\tilde{\pi}}\left(\widetilde{\sigma}, m \circ G^{-1}\right)$. 
Proof of Proposition 4.20: Let $\left(v_{n}\right)$ be a sequence in $\mathcal{M}_{\sigma}(\Sigma)$ converging to $m$ in the weak-star topology. We need to show that $\limsup _{n \rightarrow \infty} h_{\pi}\left(\sigma, v_{n}\right) \leq$ $h_{\pi}(\sigma, m)$. To see this, it suffices to show that

$$
\limsup _{n \rightarrow \infty} h_{\pi}\left(\sigma, v_{n}\right) \leq h_{\pi}(\sigma, m)+\frac{1}{k} \log t_{k}
$$

for each $k \in \mathbb{N}$, where $t_{k}$ is given as in Definition 2.14.

To prove (4.32), we fix $k \in \mathbb{N}$. Define an equivalence relation $\sim$ on $\{1, \ldots, \ell\}^{k}$ by $u \sim v$ if $S_{u}=S_{v}$. Let $\underline{u}$ denotes the equivalence class containing $u$. Denote $S_{\underline{u}}=S_{u}$. Set $\mathcal{J}=\left\{\underline{u}: u \in\{1, \ldots, \ell\}^{k}\right\}$. Let $\left(\mathcal{J}^{\mathbb{N}}, T\right)$ denote the one-sided full shift space over the alphabet $\mathcal{J}$. Let $G: \Sigma \rightarrow \mathcal{J}^{N}$ be defined by

$$
\left.\left(x_{i}\right)_{i=1}^{\infty} \mapsto \underline{\left(x_{j k+1} \cdots x_{(j+1) k}\right.}\right)_{j=0}^{\infty} .
$$

It is clear that the following diagram commutes:



That is, $T \circ G=G \circ \sigma^{k}$. It implies that $v_{n} \circ G^{-1}, m \circ G^{-1} \in \mathcal{M}_{T}\left(\mathcal{J}^{\mathbb{N}}\right)$, and

$$
\lim _{n \rightarrow \infty} v_{n} \circ G^{-1}=m \circ G^{-1} \text {. }
$$

Hence we have

$$
h\left(T, m \circ G^{-1}\right) \geq \limsup _{n \rightarrow \infty} h\left(T, v_{n} \circ G^{-1}\right),
$$

where we use the upper semicontinuity of the classical measure-theoretic entropy map on $\left(\mathcal{J}^{\mathbb{N}}, T\right)$.

Define $\tilde{\pi}: \mathcal{J}^{\mathbb{N}} \rightarrow K$ by

$$
\tilde{\pi}\left(\left(\underline{u_{i}}\right)_{i=1}^{\infty}\right)=\lim _{n \rightarrow \infty} S_{\underline{u_{1}}} \circ \cdots \circ S_{\underline{u_{n}}}(K) .
$$

Then $\tilde{\pi} \circ G=\pi$. By the assumption of AWSC (2.11) and Corollary 4.22 (considering the IFS $\left.\left\{S_{\underline{u}}: \underline{u} \in \mathcal{J}\right\}\right)$, we have

$$
\begin{aligned}
h_{\tilde{\pi}}\left(T, m \circ G^{-1}\right) & \geq h\left(T, m \circ G^{-1}\right)-\log t_{k} \\
& \geq \limsup _{n \rightarrow \infty} h\left(T, v_{n} \circ G^{-1}\right)-\log t_{k} \quad(\text { by }(4.33)) \\
& \geq \limsup _{n \rightarrow \infty} h_{\tilde{\pi}}\left(T, v_{n} \circ G^{-1}\right)-\log t_{k},
\end{aligned}
$$

where the last inequality follows from Proposition 4.1(i). Then (4.32) follows from the above inequality, together with Proposition 4.3 and the following claim:

$$
h_{\tilde{\pi}}\left(T, v \circ G^{-1}\right)=h_{\pi}\left(\sigma^{k}, v\right), \quad v \in \mathcal{M}_{\sigma}(\Sigma) .
$$


However, (4.34) just comes from Lemma 4.23, where we consider the IFS $\left\{S_{u}\right.$ : $\left.u \in\{1, \ldots, \ell\}^{k}\right\}$ rather than $\left\{S_{i}\right\}_{i=1}^{\ell}$.

\section{Some Geometric Properties of $C^{1}$ IFSs}

In this section we give some geometric properties of $C^{1}$ IFSs.

LEMMA 5.1 Let $S: U \rightarrow S(U) \subset \mathbb{R}^{d}$ be a $C^{1}$ diffeomorphism on an open set $U \subset \mathbb{R}^{d}$, and $X$ a compact subset of $U$. Let $c>1$. Then there exists $r_{0}>0$ such that

$$
c^{-1}\left\|S^{\prime}(x) \rrbracket \cdot|x-y| \leq|S(x)-S(y)| \leq c\right\| S^{\prime}(x) \| \cdot|x-y|
$$

for all $x \in X, y \in U$, with $|x-y| \leq r_{0}$, where $S^{\prime}(x)$ denotes the differential of $S$ at $x$, and $\|\cdot\|$ and $\|\cdot\|$ are defined as in (2.4). As a consequence,

$$
B\left(S(x), c^{-1} \rrbracket S^{\prime}(x) \rrbracket r\right) \subset S(B(x, r)) \subset B\left(S(x), c\left\|S^{\prime}(x)\right\| r\right)
$$

for all $x \in X$ and $0<r \leq r_{0}$.

PROOF: Let $c>1$. We only prove (5.1), for it is not hard to derive (5.2) from (5.1). Assume on the contrary that (5.1) is not true. Then there exist two sequences $\left(x_{n}\right) \subset X,\left(y_{n}\right) \subset U$ such that $x_{n} \neq y_{n}, \lim _{n \rightarrow \infty}\left|x_{n}-y_{n}\right|=0$, and for each $n \geq 1$,

$$
\begin{aligned}
& \text { either }\left|S\left(x_{n}\right)-S\left(y_{n}\right)\right| \geq c\left\|S^{\prime}\left(x_{n}\right)\right\| \cdot\left|x_{n}-y_{n}\right| \\
& \quad \text { or }\left|S\left(x_{n}\right)-S\left(y_{n}\right)\right| \leq c^{-1} \rrbracket S^{\prime}\left(x_{n}\right) \rrbracket \cdot\left|x_{n}-y_{n}\right| .
\end{aligned}
$$

Since $X$ is compact, without lost of generality, we assume that

$$
\lim _{n \rightarrow \infty} x_{n}=x=\lim _{n \rightarrow \infty} y_{n} .
$$

Write $S=\left(f_{1}, f_{2}, \ldots, f_{d}\right)^{\top}$. Then each component $f_{j}$ of $S$ is a $C^{1}$ real-valued function defined on $U$. Choose a small $\epsilon>0$ such that

$$
\left\{z \in \mathbb{R}^{d}:|z-x| \leq \epsilon \text { for some } x \in X\right\} \subset U .
$$

Take $N \in \mathbb{N}$ such that $\left|x_{n}-y_{n}\right|<\epsilon$ for $n \geq N$. By the mean value theorem, for each $n \geq N$ and $1 \leq j \leq d$, there exists $z_{n, j}$ on the segment $L_{x_{n}, y_{n}}$ connecting $x_{n}$ and $y_{n}$ such that

$$
f_{j}\left(x_{n}\right)-f_{j}\left(y_{n}\right)=\nabla f_{j}\left(z_{n, j}\right) \cdot\left(x_{n}-y_{n}\right),
$$

where $\nabla f_{j}$ denotes the gradient of $f_{j}$. Therefore $\left|S\left(x_{n}\right)-S\left(y_{n}\right)\right|=\left|M_{n}\left(x_{n}-y_{n}\right)\right|$ with $M_{n}:=\left(\nabla f_{1}\left(z_{n, 1}\right), \ldots, \nabla f_{d}\left(z_{n, d}\right)\right)^{\top}$. It follows that

$$
\llbracket M_{n} \rrbracket \cdot\left|x_{n}-y_{n}\right| \leq\left|S\left(x_{n}\right)-S\left(y_{n}\right)\right| \leq\left\|M_{n}\right\| \cdot\left|x_{n}-y_{n}\right| .
$$

Since $S$ is $C^{1}, M_{n}$ tends to $S^{\prime}(x)$ as $n \rightarrow \infty$. Thus we have $\llbracket M_{n} \rrbracket \rightarrow \llbracket S^{\prime}(x) \rrbracket$ and $\left\|M_{n}\right\| \rightarrow\left\|S^{\prime}(x)\right\|$. Meanwhile, $\llbracket S^{\prime}\left(x_{n}\right) \rrbracket \rightarrow \llbracket S^{\prime}(x) \rrbracket$ and $\left\|S^{\prime}\left(x_{n}\right)\right\| \rightarrow\left\|S^{\prime}(x)\right\|$. These limits together with (5.4) lead to a contradiction with (5.3). 
Let $\left\{S_{1}, \ldots, S_{\ell}\right\}$ be a $C^{1}$ IFS on a compact set $X \subset \mathbb{R}^{d}$. Let $\pi: \Sigma \rightarrow \mathbb{R}^{d}$ be defined as in (2.1). By Lemma 5.1, we directly have the following:

Lemma 5.2 Let $c>1$. Then there exists $r_{0}>0$ such that for any $1 \leq i \leq \ell$, $x \in \Sigma$, and $0<r<r_{0}$,

$$
B\left(S_{i}(\pi x), c^{-1} \llbracket S_{i}^{\prime}(\pi x) \rrbracket r\right) \subset S_{i}(B(\pi x, r)) \subset B\left(S_{i}(\pi x), c\left\|S_{i}^{\prime}(\pi x)\right\| r\right) .
$$

Let $\bar{\rho}, \underline{\rho}: \Sigma \rightarrow \mathbb{R}$ be defined by

$$
\bar{\rho}(x)=\left\|S_{x_{1}}^{\prime}(\pi \sigma x)\right\|, \quad \underline{\rho}(x)=\| S_{x_{1}}^{\prime}(\pi \sigma x) \rrbracket, \quad x=\left(x_{i}\right)_{i=1}^{\infty} \in \Sigma .
$$

Let $\mathcal{P}$ be the partition of $\Sigma$ defined as in (2.3). For $x \in \Sigma$, let $\mathcal{P}(x)$ denote the element in $\mathcal{P}$ that contains $x$. Then we have the following:

Lemma 5.3 Let $c>1$. Then there exists $r_{0}>0$ such that for any $z \in \Sigma$ and $0<r<r_{0}$

$$
B^{\pi}\left(z, c^{-1} \underline{\rho}(z) r\right) \cap \mathcal{P}(z) \subset B^{\pi \sigma}(z, r) \cap \mathcal{P}(z) \subset B^{\pi}(z, c \bar{\rho}(z) r) \cap \mathcal{P}(z),
$$

where $B^{\pi}(z, r)$ is defined as in (3.1).

PROOF: Let $z=\left(z_{j}\right)_{j=1}^{\infty} \in \Sigma$. Taking $i=z_{1}$ and $x=\sigma z$ in Lemma 5.2, we obtain

$$
\begin{aligned}
B\left(S_{z_{1}}(\pi \sigma z), c^{-1} \rrbracket S_{z_{1}}^{\prime}(\pi \sigma z) \| r\right) & \subset S_{z_{1}}(B(\pi \sigma z, r)) \\
& \subset B\left(S_{z_{1}}(\pi \sigma z), c\left\|S_{z_{1}}^{\prime}(\pi \sigma z)\right\| r\right) .
\end{aligned}
$$

That is,

$$
B\left(\pi z, c^{-1} \underline{\rho}(z) r\right) \subset S_{z_{1}} B(\pi \sigma z, r) \subset B(\pi z, c \bar{\rho}(z) r),
$$

where we use the fact that $S_{z_{1}}(\pi \sigma z)=\pi z$, which can be checked directly from the definition of $\pi$. Thus we have

$$
\begin{aligned}
B^{\pi}\left(\pi z, c^{-1} \underline{\rho}(z) r\right) \cap \mathcal{P}(z) & \subset \pi^{-1}\left(S_{z_{1}}(B(\pi \sigma z, r))\right) \cap \mathcal{P}(z) \\
& \subset B^{\pi}(z, c \bar{\rho}(z) r) \cap \mathcal{P}(z) .
\end{aligned}
$$

Finally, we show that

$$
\pi^{-1}\left(S_{z_{1}}(B(\pi \sigma z, r))\right) \cap \mathcal{P}(z)=B^{\pi \sigma}(z, r) \cap \mathcal{P}(z) .
$$

To see this, let $y=\left(y_{j}\right)_{j=1}^{\infty} \in \Sigma$. Then we have the following equivalent implications:

$$
\begin{aligned}
y & \in \pi^{-1}\left(S_{z_{1}}(B(\pi \sigma z, r))\right) \cap \mathcal{P}(z) \\
& \Longleftrightarrow y_{1}=z_{1}, \quad \pi y \in S_{z_{1}}(B(\pi \sigma z, r)), \\
& \Longleftrightarrow y_{1}=z_{1}, \quad S_{y_{1}}(\pi \sigma y) \in S_{z_{1}}(B(\pi \sigma z, r)), \\
& \Longleftrightarrow y_{1}=z_{1}, \quad \pi \sigma y \in B(\pi \sigma z, r), \\
& \Longleftrightarrow y_{1}=z_{1}, \quad y \in B^{\pi \sigma}(z, r), \\
& \Longleftrightarrow y \in B^{\pi \sigma}(z, r) \cap \mathcal{P}(z) .
\end{aligned}
$$

This finishes the proof of the lemma. 
LEMMA 5.4 Assume that $\left\{S_{i}\right\}_{i=1}^{\ell}$ is a weakly conformal IFS with attractor $K$. Then for any $c>1$, there exists $D>0$ such that for any $n \in \mathbb{N}, u \in\{1, \ldots, \ell\}^{n}$, and $x, y \in K$ we have

$$
D^{-1} c^{-n}\left\|S_{u}^{\prime}(x)\right\| \cdot|x-y| \leq\left|S_{u}(x)-S_{u}(y)\right| \leq D c^{n}\left\|S_{u}^{\prime}(x)\right\| \cdot|x-y|
$$

and

$$
D^{-1} c^{-n}\left\|S_{u}^{\prime}(x)\right\| \leq \operatorname{diam}\left(S_{u}(K)\right) \leq D c^{n}\left\|S_{u}^{\prime}(x)\right\| .
$$

PROOF: The results were proved in the conformal case in [18, lemma 3.5 and corollary 3.6]. A slight modification of that proof works for the weakly conformal case.

As a corollary, we have the following:

COROLlaRY 5.5 Under the assumption of Lemma 5.4, for $\alpha>0$, there is $r_{0}>0$ such that for any $0<r<r_{0}$ and $z \in K$, there exist $n \in \mathbb{N}$ and $u \in\{1, \ldots, \ell\}^{n}$ such that $S_{u}(K) \subset B(z, r)$ and

$$
\left|S_{u}(x)-S_{u}(y)\right| \geq r^{1+\alpha}|x-y|, \quad x, y \in K .
$$

Proof: Denote $a=\inf \left\{\llbracket S_{i}^{\prime}(x) \rrbracket: x \in K, 1 \leq i \leq \ell\right\}$ and $b=\sup \left\{\left\|S_{i}^{\prime}(x)\right\|:\right.$ $x \in K, 1 \leq i \leq \ell\}$. Then $0<a \leq b<1$. Choose $c$ so that

$$
1<c<b^{\frac{-\alpha}{3(2+\alpha)}} \text {. }
$$

Let $D$ be the constant in Lemma 5.4 corresponding to $c$. Take $n_{0} \in \mathbb{N}$ and $r_{0}>0$ such that

$$
\left(c^{3} b^{\frac{\alpha}{2+\alpha}}\right)^{n_{0}}<D^{-3} a b^{\frac{\alpha}{2+\alpha}}, \quad\left(1+\frac{\alpha}{2}\right) \cdot \frac{\log r_{0}}{\log a}=n_{0} .
$$

Now fix $z \in K$ and $0<r<r_{0}$. We shall show that there exist $n \in \mathbb{N}$ and $u \in\{1, \ldots, \ell\}^{n}$ such that $S_{u}(K) \subset B(z, r)$ and (5.7) holds. To see this, take $\omega=\left(\omega_{i}\right)_{i=1}^{\infty} \in \Sigma$ such that $z=\pi \omega$, where $\pi$ is defined as in (2.1). Let $n$ be the unique integer such that

$$
\left\|S_{\omega_{1} \cdots \omega_{n}}^{\prime}\left(\pi \sigma^{n} \omega\right)\right\|<r^{1+\frac{\alpha}{2}} \leq\left\|S_{\omega_{1} \cdots \omega_{n-1}}^{\prime}\left(\pi \sigma^{n-1} \omega\right)\right\| .
$$

It follows that $a^{n}<r^{1+\alpha / 2} \leq b^{n-1}$, which together with (5.9) forces that

$$
n>n_{0} \quad \text { and } \quad c^{3 n}<D^{-3} a r^{-\frac{\alpha}{2}} \text {. }
$$

To see (5.11), we first assume on the contrary that $n \leq n_{0}$. Then

$$
a^{n} \geq a^{n_{0}}=a^{\left(1+\frac{\alpha}{2}\right) \frac{\log r_{0}}{\log \alpha}}=r_{0}^{1+\frac{\alpha}{2}}>r^{1+\frac{\alpha}{2}},
$$

contradicting the fact $a^{n}<r^{1+\alpha / 2}$. Hence $n>n_{0}$. To see $c^{3 n}<D^{-3} a r^{-\alpha / 2}$, note that

$$
\begin{aligned}
c^{3 n} r^{\frac{\alpha}{2}} & \leq c^{3 n} b^{(n-1) \frac{\alpha}{2+\alpha}} \quad \quad\left(\text { using } r^{1+\frac{\alpha}{2}} \leq b^{n-1}\right) \\
& \leq\left(c^{3} b^{\frac{\alpha}{2+\alpha}}\right)^{n} b^{-\frac{\alpha}{2+\alpha}} \leq
\end{aligned}
$$




$$
\begin{aligned}
& \leq\left(c^{3} b^{\frac{\alpha}{2+\alpha}}\right)^{n_{0}} b^{-\frac{\alpha}{2+\alpha}} \quad \text { (using } n>n_{0} \text { and (5.8)) } \\
& \leq D^{-3} a \quad \text { (by (5.9)). }
\end{aligned}
$$

This completes the proof of (5.11).

By (5.6), we have

$$
\operatorname{diam} S_{\omega_{1} \cdots \omega_{n}}(K) \leq D c^{n}\left\|S_{\omega_{1} \cdots \omega_{n}}^{\prime}\left(\pi \sigma^{n} \omega\right)\right\| \leq D c^{n} r^{1+\frac{\alpha}{2}}<r .
$$

Since $z \in S_{\omega_{1} \cdots \omega_{n}}(K)$, the above inequality implies $S_{\omega_{1} \cdots \omega_{n}}(K) \subset B(z, r)$. By (5.6) again, we have

$$
\left\|S_{u}^{\prime}(x)\right\| \geq D^{-2} c^{-2 n}\left\|S_{u}^{\prime}(y)\right\| \quad \forall u \in\{1, \ldots, \ell\}^{n}, \quad \forall x, y \in K
$$

By Lemma 5.4, we have for $x, y \in K$,

$$
\begin{aligned}
& \left|S_{\omega_{1} \cdots \omega_{n}}(x)-S_{\omega_{1} \cdots \omega_{n}}(y)\right| \\
& \quad \geq D^{-1} c^{-n}\left\|S_{\omega_{1} \cdots \omega_{n}}^{\prime}(x)\right\| \cdot|x-y| \\
& \quad \geq D^{-3} c^{-3 n}\left\|S_{\omega_{1} \cdots \omega_{n}}^{\prime}\left(\pi \sigma^{n} \omega\right)\right\| \cdot|x-y| \\
& \quad \geq D^{-3} c^{-3 n}\left\|S_{\omega_{1} \cdots \omega_{n-1}}^{\prime}\left(\pi \sigma^{n-1} \omega\right)\right\| \llbracket S_{\omega_{n}}^{\prime}\left(\pi \sigma^{n} \omega\right) \rrbracket \cdot|x-y| \\
& \quad \geq D^{-3} c^{-3 n} a r^{1+\frac{\alpha}{2}}|x-y| \\
& \quad \geq r^{1+\alpha}|x-y|
\end{aligned}
$$

Hence the corollary follows by taking $u=\omega_{1} \cdots \omega_{n}$.

Proposition 5.6 Let $\left\{S_{i}\right\}_{i=1}^{\ell}$ be a $C^{1}$ IFS with attractor $K$. Assume that $K$ is not a singleton. Then:

(i) For any $m \in \mathcal{M}_{\sigma}(\Sigma)$, we have for $m$-a.e. $x=\left(x_{i}\right)_{i=1}^{\infty} \in \Sigma$,

$$
\begin{gathered}
\liminf _{n \rightarrow \infty} \frac{\log \operatorname{diam} S_{x_{1} \cdots x_{n}}(K)}{n} \geq-\bar{\lambda}(x), \\
\limsup _{n \rightarrow \infty} \frac{\log \operatorname{diam} S_{x_{1} \cdots x_{n}}(K)}{n} \leq-\underline{\lambda}(x),
\end{gathered}
$$

where $\underline{\lambda}, \bar{\lambda}$ are defined as in Definition 2.5. In particular, if $\left\{S_{i}\right\}_{i=1}^{\ell}$ is $m$-conformal, then for $m$-a.e. $x=\left(x_{i}\right)_{i=1}^{\infty} \in \Sigma$,

$$
\lim _{n \rightarrow \infty} \frac{\log \operatorname{diam} S_{x_{1} \cdots x_{n}}(K)}{n}=-\lambda(x) .
$$

(ii) If $\left\{S_{i}\right\}_{i=1}^{\ell}$ is weakly conformal, then it is $m$-conformal for each $m \in$ $\mathcal{M}_{\sigma}(\Sigma)$.

Proof: We first prove (i). Take $c>1$ so small that $c \sup _{x \in \Sigma} \bar{\rho}(x)<1$. Let $r_{0}>0$ be given as in Lemma 5.2. Let $x=\left(x_{i}\right)_{i=1}^{\infty} \in \Sigma$. Applying Lemma 5.2 repeatedly, we have

$$
S_{x_{1} \cdots x_{n}}\left(B\left(\pi \sigma^{n} x, r_{0}\right)\right) \subset B\left(\pi x, c^{n} \bar{\rho}(x) \cdots \bar{\rho}\left(\sigma^{n-1} x\right) r_{0}\right) .
$$


Since $\left\{S_{i}\right\}_{i=1}^{\ell}$ is contractive, there is a constant $k$ such that

$$
S_{x_{n+1} \cdots x_{n+k}}(K) \subset B\left(\pi \sigma^{n} x, r_{0}\right) .
$$

This together with (5.13) yields

$$
\begin{aligned}
\operatorname{diam} S_{x_{1} \cdots x_{n+k}}(K) & \leq \operatorname{diam} S_{x_{1} \cdots x_{n}}\left(B\left(\pi \sigma^{n} x, r_{0}\right)\right) \\
& \leq 2 c^{n} \bar{\rho}(x) \cdots \bar{\rho}\left(\sigma^{n-1} x\right) r_{0} .
\end{aligned}
$$

Since $K$ is not a singleton, there exists $0<r_{1}<r_{0}$ such that for each $z \in K$, there exists $w \in K$ such that $r_{1} \leq|z-w| \leq r_{0}$. Indeed, to obtain $r_{1}$, one chooses an integer $n_{0}$ large enough such that $\sup _{u \in \Sigma_{n_{0}}} \operatorname{diam} S_{u}(K) \leq r_{0}$ and then sets

$$
r_{1}=\frac{1}{2} \inf _{u \in \Sigma_{n_{0}}} \operatorname{diam} S_{u}(K)
$$

For each such pair $(z, w)$, applying (5.1) repeatedly yields

$$
\begin{aligned}
\operatorname{diam} S_{x_{1} \cdots x_{n}}(K) & \geq\left|S_{x_{1} \cdots x_{n}}(z)-S_{x_{1} \cdots x_{n}}(w)\right| \\
& \geq r_{1} c^{-n} \prod_{j=1}^{n} \llbracket S_{x_{j}}^{\prime}\left(S_{x_{j+1} \cdots x_{n}}(z) \rrbracket .\right.
\end{aligned}
$$

Hence by taking $z=\pi \sigma^{n} x$, we have

$$
\operatorname{diam} S_{x_{1} \cdots x_{n}}(K) \geq r_{1} c^{-n} \underline{\rho}(x) \cdots \underline{\rho}\left(\sigma^{n-1} x\right) .
$$

Denote

$$
g_{*}(x)=\liminf _{n \rightarrow \infty} \frac{\log \operatorname{diam} S_{x_{1} \cdots x_{n}}(K)}{n}
$$

and

$$
g^{*}(x)=\limsup _{n \rightarrow \infty} \frac{\log \operatorname{diam} S_{x_{1} \cdots x_{n}}(K)}{n} .
$$

It is clear that $g_{*}(x)=g_{*}(\sigma x)$ and $g^{*}(x)=g^{*}(\sigma x)$. Let $\mathcal{I}$ denote the $\sigma$-algebra $\left\{B \in \mathcal{B}(\Sigma): \sigma^{-1} B=B\right\}$. Then by (5.15), the Birkhoff ergodic theorem, and theorem 34.2 in [7], we have for $m$-a.e. $x \in \Sigma$,

$$
\begin{aligned}
g_{*}(x) & =\mathbf{E}_{m}\left(g_{*} \mid \mathcal{I}\right)(x) \\
& \geq \mathbf{E}_{m}\left(\lim _{n \rightarrow \infty} \frac{-n \log c+\sum_{i=0}^{n-1} \log \underline{\rho} \circ \sigma^{-i}}{n} \mid \mathcal{I}\right)(x) \\
& =-\log c+\lim _{n \rightarrow \infty} \frac{1}{n} \sum_{i=0}^{n-1} \mathbf{E}_{m}\left(\log \underline{\rho} \circ \sigma^{-i} \mid \mathcal{I}\right)(x) \\
& =-\log c+\mathbf{E}_{m}(\log \underline{\rho} \mid \mathcal{I})(x)
\end{aligned}
$$


and similarly by (5.14),

$$
g^{*}(x) \leq \log c+\mathbf{E}_{m}(\log \bar{\rho} \mid \mathcal{I})(x) .
$$

For $p \in \mathbb{N}$, write

$$
A_{p}(x)=\log \rrbracket S_{x_{1} \cdots x_{p}}^{\prime}\left(\pi \sigma^{p} x\right) \rrbracket \quad \text { and } \quad A_{p}^{*}(x)=\log \left\|S_{x_{1} \cdots x_{p}}^{\prime}\left(\pi \sigma^{p} x\right)\right\| .
$$

Consider the IFS $\left\{S_{i_{1} \cdots i_{p}}: 1 \leq i_{j} \leq \ell, 1 \leq j \leq p\right\}$ rather than $\left\{S_{i}\right\}_{i=1}^{\ell}$. Then (5.16) and (5.17) can be replaced by

$$
g_{*}(x) \geq-\log c+\frac{1}{p} \mathbf{E}_{m}\left(A_{p} \mid \mathcal{I}_{p}\right)(x), \quad g^{*}(x) \leq \log c+\frac{1}{p} \mathbf{E}_{m}\left(A_{p}^{*} \mid \mathcal{I}_{p}\right)(x),
$$

where $\mathcal{I}_{p}:=\left\{B \in \mathcal{B}(\Sigma): \sigma^{-p} B=B\right\}$. Taking the conditional expectation with respect to $\mathcal{I}$ in the above inequalities and noting that $g_{*}, g^{*}$ are $\sigma$-invariant, we obtain

$$
\begin{aligned}
& g_{*}(x) \geq-\log c+\frac{1}{p} \mathbf{E}_{m}\left(A_{p} \mid \mathcal{I}\right)(x), \\
& g^{*}(x) \leq \log c+\frac{1}{p} \mathbf{E}_{m}\left(A_{p}^{*} \mid \mathcal{I}\right)(x) .
\end{aligned}
$$

Since $A_{p}(x)$ is sup-additive (i.e., $A_{p+q}(x) \geq A_{p}(x)+A_{q}\left(\sigma^{p} x\right)$ ) and $A_{p}^{*}(x)$ is sub-additive (i.e., $A_{p+q}^{*}(x) \leq A_{p}^{*}(x)+A_{q}^{*}\left(\sigma^{p} x\right)$ ), by Kingman's sub-additive ergodic theorem (cf. [63]), we have

$$
\lim _{p \rightarrow \infty} \frac{A_{p}(x)}{p}=-\bar{\lambda}(x), \quad \lim _{p \rightarrow \infty} \frac{A_{p}^{*}(x)}{p}=-\underline{\lambda}(x)
$$

almost everywhere and in $L^{1}$. Hence letting $c \rightarrow 1$ and $p \rightarrow \infty$ in (5.18) and using theorem 34.2 in [7], we obtain that $g_{*}(x) \geq-\bar{\lambda}(x)$ and $g^{*}(x) \leq-\underline{\lambda}(x)$ almost everywhere. This finishes the proof of (i).

To see (ii), assume that $\left\{S_{i}\right\}_{i=1}^{\ell}$ is weakly conformal and $m \in \mathcal{M}_{\sigma}(\Sigma)$. Then $\left|A_{p}(x)-A_{p}^{*}(x)\right| / p$ converges to 0 uniformly as $p$ tends to infinity. This together with (5.19) yields $\bar{\lambda}(x)=\underline{\lambda}(x)$ for $m$-a.e. $x \in \Sigma$. This proves (ii).

\section{Estimates for Local Dimensions of Invariant Measures for $C^{1}$ IFSs}

In this section, we prove a general version of Theorem 2.6, which is also needed in the proof of Theorem 2.11. Let $\left\{T_{i}\right\}_{i=1}^{\ell}$ be a $C^{1}$ IFS on $\mathbb{R}^{d}$ and $\left\{S_{i}\right\}_{i=1}^{\ell}$ a $C^{1}$ IFS on $\mathbb{R}^{k}$. Let $\phi: \Sigma \rightarrow \mathbb{R}^{d}$ and $\pi: \Sigma \rightarrow \mathbb{R}^{k}$ denote the canonical projections associated with $\left\{T_{i}\right\}_{i=1}^{\ell}$ and $\left\{S_{i}\right\}_{i=1}^{\ell}$, respectively. Let $\eta$ and $\xi$ be two partitions of $\Sigma$ defined, respectively, by

$$
\eta=\left\{\phi^{-1}(z): z \in \mathbb{R}^{d}\right\}, \quad \xi=\sigma^{-1} \eta
$$

Let $\mathcal{P}$ be the partition of $\Sigma$ given as in (2.3), and let $\bar{\rho}(x), \underline{\rho}(x)$ be defined as in (5.5). Applying Lemma 5.3 to the IFS $\left\{S_{i}\right\}_{i=1}^{\ell}$, we have for any $c>1$ that there 
exist $0<\delta<c-1$ and $r_{0}>0$ such that for any $r \in\left(0, r_{0}\right)$ and $x \in \Sigma$,

$$
\begin{aligned}
B^{\pi}\left(x,(c-\delta)^{-1} \underline{\rho}(x) r\right) \cap \mathcal{P}(x) & \subset B^{\pi \sigma}(x, r) \cap \mathcal{P}(x) \\
& \subset B^{\pi}(x,(c-\delta) \bar{\rho}(x) r) \cap \mathcal{P}(x) .
\end{aligned}
$$

The following technical proposition is important in our proof.

Proposition 6.1 Let $m \in \mathcal{M}_{\sigma}(\Sigma)$ and $c>1$. Let $\delta, r_{0}$ be given as above. Then there exists $\Lambda \subset \Sigma$ with $m(\Lambda)=1$ such that for all $x \in \Lambda$ and $r \in\left(0, r_{0}\right)$,

$$
\frac{m_{x}^{\eta}\left(B^{\pi}(x, c \bar{\rho}(x) r) \cap \mathcal{P}(x)\right)}{m_{\sigma x}^{\eta}\left(B^{\pi}(\sigma x, r)\right)} \geq f(x) \cdot \frac{m_{x}^{\xi}\left(B^{\pi \sigma}(x, r) \cap \mathcal{P}(x)\right)}{m_{x}^{\xi}\left(B^{\pi \sigma}(x, r)\right)}
$$

and

$$
\begin{aligned}
& \frac{m_{x}^{\eta}\left(B^{\pi}\left(x, c^{-1} \underline{\rho}(x) r\right) \cap \mathcal{P}(x)\right)}{m_{\sigma x}^{\eta}\left(B^{\pi}(\sigma x, r)\right)} \leq \\
& f(x) \cdot \frac{m_{x}^{\xi}\left(B^{\pi \sigma}(x,(1-c \delta / 2) r) \cap \mathcal{P}(x)\right)}{m_{x}^{\xi}\left(B^{\pi \sigma}(x,(1-c \delta / 2) r)\right)},
\end{aligned}
$$

where $f:=\sum_{A \in \mathcal{P}} \chi_{A} \frac{\mathbf{E}_{m}\left(\chi_{A} \mid \phi^{-1} \gamma\right)}{\mathbf{E}_{m}\left(\chi_{A} \mid \sigma^{-1} \phi^{-1} \gamma\right)}, \gamma=\mathcal{B}\left(\mathbb{R}^{d}\right)$.

Proof: Write $R_{t, x}(z)=T_{x_{1}}^{-1} B\left(T_{x_{1}} z, t\right)$ for $t>0, x=\left(x_{i}\right)_{i=1}^{\infty} \in \Sigma$, and $z \in \mathbb{R}^{d}$. It is direct to check that

$$
\sigma^{-1} \phi^{-1} R_{t, x}(\phi \sigma x) \cap \mathcal{P}(x)=B^{\phi}(x, t) \cap \mathcal{P}(x) .
$$

Hence for $m$-a.e. $x$,

$$
\begin{aligned}
\frac{m\left(\phi^{-1} R_{t, x}(\phi \sigma x)\right)}{m\left(B^{\phi}(x, t)\right)} & =\frac{m\left(B^{\phi}(x, t) \cap \mathcal{P}(x)\right)}{m\left(B^{\phi}(x, t)\right)} \cdot \frac{m\left(\phi^{-1} R_{t, x}(\phi \sigma x)\right)}{m\left(B^{\phi}(x, t) \cap \mathcal{P}(x)\right)} \\
& =\frac{m\left(B^{\phi}(x, t) \cap \mathcal{P}(x)\right)}{m\left(B^{\phi}(x, t)\right)} \cdot \frac{m\left(\sigma^{-1} \phi^{-1} R_{t, x}(\phi \sigma x)\right)}{m\left(\sigma^{-1} \phi^{-1} R_{t, x}(\phi \sigma x) \cap \mathcal{P}(x)\right)}
\end{aligned}
$$

Letting $t \rightarrow 0$ and applying Proposition 3.5 and Remark 3.6, we have

$$
\lim _{t \rightarrow 0} \frac{m\left(\phi^{-1} R_{t, x}(\phi \sigma x)\right)}{m\left(B^{\phi}(x, t)\right)}=\sum_{A \in \mathcal{P}} \chi_{A}(x) \frac{\mathbf{E}_{m}\left(\chi_{A} \mid \phi^{-1} \gamma\right)(x)}{\mathbf{E}_{m}\left(\chi_{A} \mid \sigma^{-1} \phi^{-1} \gamma\right)(x)}=: f(x) .
$$

for $m$-a.e. $x$.

Let $\tilde{\Lambda}$ denote the set of $x \in \Sigma$ such that the following properties hold:

(1) $\lim _{t \rightarrow 0} \frac{m\left(B^{\phi}(x, t) \cap \mathcal{P}(x)\right)}{m\left(B^{\phi}(x, t)\right)}=\sum_{A \in \mathcal{P}} \chi_{A} \mathbf{E}_{m}\left(\chi_{A} \mid \phi^{-1} \gamma\right)(x)>0$.

(2) $\lim _{t \rightarrow 0} \frac{m\left(\sigma^{-1} \phi^{-1} R_{t, x}(\phi \sigma x) \cap \mathcal{P}(x)\right)}{m\left(\sigma^{-1} \phi^{-1} R_{t, x}(\phi \sigma x)\right)}=\sum_{A \in \mathcal{P}} \chi_{A} \mathbf{E}_{m}\left(\chi_{A} \mid \sigma^{-1} \phi^{-1} \gamma\right)(x)$

$$
>0 \text {. }
$$


(3) For all $q \in \mathbb{Q}^{+}$,

$$
\begin{aligned}
& m_{x}^{\eta}\left(B^{\pi}(x, q) \cap \mathcal{P}(x)\right) \geq \limsup _{t \rightarrow 0} \frac{m\left(B^{\pi}(x, q) \cap \mathcal{P}(x) \cap B^{\phi}(x, t)\right)}{m\left(B^{\phi}(x, t)\right)}, \\
& m_{x}^{\eta}\left(U^{\pi}(x, q) \cap \mathcal{P}(x)\right) \leq \liminf _{t \rightarrow 0} \frac{m\left(B^{\pi}(x, q) \cap \mathcal{P}(x) \cap B^{\phi}(x, t)\right)}{m\left(B^{\phi}(x, t)\right)}, \\
& m_{x}^{\xi}\left(B^{\pi \sigma}(x, q) \cap \mathcal{P}(x)\right) \geq \limsup _{t \rightarrow 0} \frac{m\left(B^{\pi \sigma}(x, q) \cap \mathcal{P}(x) \cap \sigma^{-1} \phi^{-1} R_{t, x}(\phi \sigma x)\right)}{m\left(\sigma^{-1} \phi^{-1} R_{t, x}(\phi \sigma x)\right)}, \\
& m_{x}^{\xi}\left(U^{\pi \sigma}(x, q) \cap \mathcal{P}(x)\right) \leq \liminf _{t \rightarrow 0} \frac{m\left(B^{\pi \sigma}(x, q) \cap \mathcal{P}(x) \cap \sigma^{-1} \phi^{-1} R_{t, x}(\phi \sigma x)\right)}{m\left(\sigma^{-1} \phi^{-1} R_{t, x}(\phi \sigma x)\right)},
\end{aligned}
$$

where

$$
U^{\pi}(x, q):=\pi^{-1} U(\pi x, q), \quad U^{\pi \sigma}(x, q):=\sigma^{-1} \pi^{-1} U(\pi \sigma x, q),
$$

and $U(z, q)$ denotes the open ball in $\mathbb{R}^{k}$ of radius $q$ centered at $z$.

$$
\text { (4) } \lim _{t \rightarrow 0} \frac{m\left(\phi^{-1} R_{t, x}(\phi \sigma x)\right)}{m\left(B^{\phi}(x, t)\right)}=f(x) \text {. }
$$

Then we have $m(\tilde{\Lambda})=1$ by Proposition 3.5, Lemma 3.7, Remarks 3.6 and 3.8, and (6.5).

Now let $\Lambda=\tilde{\Lambda} \cap \sigma^{-1} \tilde{\Lambda}$. Then $m(\Lambda)=1$. Fix $x \in \Lambda$ and $r \in\left(0, r_{0}\right)$. Let $q_{1} \in \mathbb{Q}^{+} \cap(r, c r /(c-\delta))$. Choose $q_{2}, q_{3} \in \mathbb{Q}^{+}$such that $q_{1}<q_{2}<c r /(c-\delta)$ and $q_{2}(c-\delta) \bar{\rho}(x)<q_{3}<c \bar{\rho}(x) r$. By (6.1), we have $B^{\pi}\left(x, q_{3}\right) \cap \mathcal{P}(x) \supset$ $B^{\pi \sigma}\left(x, q_{2}\right) \cap \mathcal{P}(x)$. This together with (6.4) yields

$$
\begin{aligned}
& B^{\pi}\left(x, q_{3}\right) \cap \mathcal{P}(x) \cap B^{\phi}(x, t) \supset \\
& \quad B^{\pi \sigma}\left(x, q_{2}\right) \cap \mathcal{P}(x) \cap \sigma^{-1} \phi^{-1} R_{t, x}(\phi \sigma x) .
\end{aligned}
$$

Hence we have

$$
\begin{aligned}
& \frac{m_{x}^{\eta}\left(B^{\pi}(x, c \bar{\rho}(x) r) \cap \mathcal{P}(x)\right)}{m_{\sigma x}^{\eta}\left(B^{\pi}(\sigma x, r)\right)} \\
& \geq \frac{m_{x}^{\eta}\left(B^{\pi}\left(x, q_{3}\right) \cap \mathcal{P}(x)\right)}{m_{\sigma x}^{\eta}\left(U^{\pi \sigma}\left(x, q_{1}\right)\right)} \\
& \geq \frac{\limsup _{t \rightarrow 0} m\left(B^{\pi}\left(x, q_{3}\right) \cap \mathcal{P}(x) \cap B^{\phi}(x, t)\right) / m\left(B^{\phi}(x, t)\right)}{\liminf _{t \rightarrow 0} m\left(B^{\pi}\left(\sigma x, q_{1}\right) \cap \phi^{-1} R_{t, x}(\phi \sigma x)\right) / m\left(\phi^{-1} R_{t, x}(\phi \sigma x)\right)} \\
& \quad(\operatorname{by~Lemma~} 3.7 \text { and Remark 3.8) } \\
& \geq \lim _{t \rightarrow 0} \frac{m\left(\phi^{-1} R_{t, x}(\phi \sigma x)\right)}{m\left(B^{\phi}(x, t)\right)} \cdot \limsup _{t \rightarrow 0} \frac{m\left(B^{\pi}\left(x, q_{3}\right) \cap \mathcal{P}(x) \cap B^{\phi}(x, t)\right)}{m\left(\sigma^{-1} B^{\pi}\left(\sigma x, q_{1}\right) \cap \sigma^{-1} \phi^{-1} R_{t, x}(\phi \sigma x)\right)} \\
&= \lim _{t \rightarrow 0} \frac{m\left(\phi^{-1} R_{t, x}(\phi \sigma x)\right)}{m\left(B^{\phi}(x, t)\right)} \cdot \limsup _{t \rightarrow 0} \frac{m\left(B^{\pi}\left(x, q_{3}\right) \cap \mathcal{P}(x) \cap B^{\phi}(x, t)\right)}{m\left(B^{\pi \sigma}\left(x, q_{1}\right) \cap \sigma^{-1} \phi^{-1} R_{t, x}(\phi \sigma x)\right)} .
\end{aligned}
$$


Denote

$$
\begin{aligned}
X_{t} & :=m\left(B^{\pi \sigma}\left(x, q_{2}\right) \cap \mathcal{P}(x) \cap \sigma^{-1} \phi^{-1} R_{t, x}(\phi \sigma x)\right), \\
Y_{t} & :=m\left(B^{\pi \sigma}\left(x, q_{1}\right) \cap \sigma^{-1} \phi^{-1} R_{t, x}(\phi \sigma x)\right), \\
Z_{t} & :=m\left(\sigma^{-1} \phi^{-1} R_{t, x}(\phi \sigma x)\right) .
\end{aligned}
$$

Using property (4), we have

$$
\begin{aligned}
& \frac{m_{x}^{\eta}\left(B^{\pi}(x, c \bar{\rho}(x) r) \cap \mathcal{P}(x)\right)}{m_{\sigma x}^{\eta}\left(B^{\pi}(\sigma x, r)\right)} \\
& \geq f(x) \cdot \limsup _{t \rightarrow 0} \frac{m\left(B^{\pi}\left(x, q_{3}\right) \cap \mathcal{P}(x) \cap B^{\phi}(x, t)\right)}{m\left(B^{\pi \sigma}\left(x, q_{1}\right) \cap \sigma^{-1} \phi^{-1} R_{t, x}(\phi \sigma x)\right)} \\
& \geq f(x) \cdot \limsup _{t \rightarrow 0} X_{t} / Y_{t} \quad(\text { by }(6.6)) \\
& \geq f(x) \cdot \limsup _{t \rightarrow 0} \frac{X_{t} / Z_{t}}{Y_{t} / Z_{t}} \geq f(x) \cdot \frac{\liminf _{t \rightarrow 0} X_{t} / Z_{t}}{\limsup _{t \rightarrow 0} Y_{t} / Z_{t}} \\
& \geq f(x) \cdot \frac{m_{x}^{\xi}\left(U^{\pi \sigma}\left(x, q_{1}\right) \cap \mathcal{P}(x)\right)}{m_{x}^{\xi}\left(B^{\pi \sigma}\left(x, q_{1}\right)\right)} \quad(\text { by Lemma 3.7 and Remark 3.8) } \\
& \geq f(x) \cdot \frac{m_{x}^{\xi}\left(B^{\pi \sigma}(x, r) \cap \mathcal{P}(x)\right)}{m_{x}^{\xi}\left(B^{\pi \sigma}\left(x, q_{1}\right)\right)} .
\end{aligned}
$$

Letting $q_{1} \downarrow r$, we obtain (6.2). Inequality (6.3) follows from an analogous argument.

Let $(\phi, \pi)$ denote the map $\Sigma \rightarrow \mathbb{R}^{d} \times \mathbb{R}^{k}, x \mapsto(\phi x, \pi x)$. It is easy to see that $(\phi, \pi)$ is the canonical projection with respect to the direct product of $\left\{T_{i}\right\}_{i=1}^{\ell}$ and $\left\{S_{i}\right\}_{i=1}^{\ell}$. In the following we give a general version of Theorem 2.6.

THEOREM 6.2 Let $m \in \mathcal{M}_{\sigma}(\Sigma)$. Then for $m$-a.e. $x \in \Sigma$, we have

$$
\limsup _{r \rightarrow 0} \frac{\log m_{x}^{\eta}\left(B^{\pi}(x, r)\right)}{\log r} \leq \frac{\mathbf{E}_{m}(g \mid \mathcal{I})(x)}{-\underline{\lambda}(x)}
$$

and

$$
\liminf _{r \rightarrow 0} \frac{\log m_{x}^{\eta}\left(B^{\pi}(x, r)\right)}{\log r} \geq \frac{\mathbf{E}_{m}(g \mid \mathcal{I})(x)}{-\bar{\lambda}(x)}
$$

where

$$
\begin{aligned}
g:= & \mathbf{I}_{m}\left(\mathcal{P} \mid \sigma^{-1} \phi^{-1} \mathcal{B}\left(\mathbb{R}^{d}\right)\right)-\mathbf{I}_{m}\left(\mathcal{P} \mid \phi^{-1} \mathcal{B}\left(\mathbb{R}^{d}\right)\right) \\
& +\mathbf{I}_{m}\left(\mathcal{P} \mid(\phi, \sigma)^{-1} \mathcal{B}\left(\mathbb{R}^{d} \times \mathbb{R}^{k}\right)\right)-\mathbf{I}_{m}\left(\mathcal{P} \mid \sigma^{-1}(\phi, \pi)^{-1} \mathcal{B}\left(\mathbb{R}^{d} \times \mathbb{R}^{k}\right)\right),
\end{aligned}
$$


and $\bar{\lambda}(x), \underline{\lambda}(x)$ denote the upper and lower Lyapunov exponents of $\left\{S_{i}\right\}_{i=1}^{\ell}$ at $x$ (see Definition 2.5). In particular, if $\left\{S_{i}\right\}_{i=1}^{\ell}$ is $m$-conformal, we have

$$
\lim _{r \rightarrow 0} \frac{\log m_{x}^{\eta}\left(B^{\pi}(x, r)\right)}{\log r}=\frac{h_{(\phi, \pi)}(\sigma, m, x)-h_{\phi}(\sigma, m, x)}{\lambda(x)} .
$$

PROOF: It suffices to prove (6.7) and (6.8). For brevity, we prove only (6.7). The proof of (6.8) is analogous.

We first prove the inequality

$$
\limsup _{r \rightarrow 0} \frac{\log m_{x}^{\eta}\left(B^{\pi}(x, r)\right)}{\log r} \leq \frac{\mathbf{E}_{m}(g \mid \mathcal{I})(x)}{\mathbf{E}_{m}(\log \bar{\rho} \mid \mathcal{I})(x)} \quad m \text {-a.e. }
$$

where $\bar{\rho}(x)=\left\|S_{x_{1}}^{\prime}(\sigma x)\right\|$ for $x=\left(x_{i}\right)_{i=1}^{\infty}$. To see this, let $c>1$ so that

$$
c \sup _{x \in \Sigma} \bar{\rho}(x)<1 .
$$

Let $r_{0}$ and $f$ be given as in Proposition 6.1. For $n \in \mathbb{N}$ and $x \in \Sigma$, define

$$
\bar{\rho}_{n}(x)=\bar{\rho}(x) \bar{\rho}(\sigma x) \cdots \bar{\rho}\left(\sigma^{n-1} x\right) .
$$

Write

$$
\begin{aligned}
H_{n}(x) & :=\log \frac{m_{x}^{\eta}\left(B^{\pi}\left(x, c^{n} \bar{\rho}_{n}(x) r_{0}\right)\right)}{m_{\sigma x}^{\eta}\left(B^{\pi}\left(\sigma x, c^{n-1} \bar{\rho}_{n-1}(\sigma x) r_{0}\right)\right)} \\
G_{n}(x) & :=\log \frac{m_{x}^{\eta}\left(B^{\pi}\left(x, c^{n} \bar{\rho}_{n}(x) r_{0}\right) \cap \mathcal{P}(x)\right)}{m_{x}^{\eta}\left(B^{\pi}\left(x, c^{n} \bar{\rho}_{n}(x) r_{0}\right)\right)} \\
W_{n}(x) & :=\log \frac{m_{x}^{\xi}\left(B^{\pi \sigma}\left(x, c^{n-1} \bar{\rho}_{n-1}(\sigma x) r_{0}\right) \cap \mathcal{P}(x)\right)}{m_{x}^{\xi}\left(B^{\pi \sigma}\left(x, c^{n-1} \bar{\rho}_{n-1}(\sigma x) r_{0}\right)\right)} .
\end{aligned}
$$

Then by Proposition 6.1 we have for $m$-a.e. $x, H_{n}(x)+G_{n}(x) \geq \log f(x)+$ $W_{n}(x)$, that is,

$$
H_{n}(x) \geq \log f(x)-G_{n}(x)+W_{n}(x)
$$

However,

$$
\log m_{x}^{\eta}\left(B^{\pi}\left(x, c^{n} \bar{\rho}_{n}(x) r_{0}\right)\right)=\sum_{j=0}^{n-1} H_{n-j}\left(\sigma^{j} x\right)+\log m_{\sigma^{n} x}^{\eta}\left(B^{\pi}\left(\sigma^{n} x, r_{0}\right)\right) .
$$

Hence for $m$-a.e. $x$,

$$
\begin{aligned}
& \frac{\log m_{x}^{\eta}\left(B^{\pi}\left(x, c^{n} \bar{\rho}_{n}(x) r_{0}\right)\right)}{n} \\
& \geq \frac{1}{n} \sum_{j=0}^{n-1}\left[\log f\left(\sigma^{j} x\right)-G_{n-j}\left(\sigma^{j} x\right)+W_{n-j}\left(\sigma^{j} x\right)\right] \\
& \quad+\frac{1}{n} \log m_{\sigma^{n} x}^{\eta}\left(B^{\pi}\left(\sigma^{n} x, r_{0}\right)\right)
\end{aligned}
$$


Note that by Proposition 3.5,

$$
\begin{aligned}
G_{n} \rightarrow G & :=-\mathbf{I}_{m}\left(\mathcal{P} \mid \hat{\eta} \vee \pi^{-1} \mathcal{B}\left(\mathbb{R}^{k}\right)\right), \\
W_{n} \rightarrow W & :=-\mathbf{I}_{m}\left(\mathcal{P} \mid \sigma^{-1} \hat{\eta} \vee \sigma^{-1} \pi^{-1} \mathcal{B}\left(\mathbb{R}^{k}\right)\right)
\end{aligned}
$$

pointwise and in $L^{1}$. By Lemma 4.13 and Proposition 3.9, we have for $m$-a.e. $x$,

$$
\begin{aligned}
\liminf _{n \rightarrow \infty} \frac{\log m_{x}^{\eta}\left(B^{\pi}\left(x, c^{n} \bar{\rho}_{n}(x) r_{0}\right)\right)}{n} & \geq \mathbf{E}_{m}((\log f-G+W) \mid \mathcal{I})(x) \\
& =\mathbf{E}_{m}(g \mid \mathcal{I})(x) .
\end{aligned}
$$

In the meantime, by the Birkhoff ergodic theorem, we have

$$
\lim _{n \rightarrow \infty} \frac{1}{n} \log \left(c^{n} \bar{\rho}_{n}(x) r_{0}\right)=\log c+\mathbf{E}_{m}(\log \bar{\rho} \mid \mathcal{I})(x) \quad m \text {-a.e. }
$$

Hence we have

$$
\begin{aligned}
\underset{r \rightarrow 0}{\limsup } & \frac{\log m_{x}^{\eta}\left(B^{\pi}(x, r)\right)}{\log r} \\
& =\limsup _{n \rightarrow \infty} \frac{\log m_{x}^{\eta}\left(B^{\pi}\left(x, c^{n} \bar{\rho}_{n}(x) r_{0}\right)\right)}{\log \left(c^{n} \bar{\rho}_{n}(x) r_{0}\right)} \\
& \leq \frac{\mathbf{E}_{m}(g \mid \mathcal{I})(x)}{\log c+\mathbf{E}_{m}(\log \bar{\rho} \mid \mathcal{I})(x)} .
\end{aligned}
$$

Taking $c \rightarrow 1$, we obtain (6.9).

Let $q \in \mathbb{N}$. Considering the IFS $\left\{T_{i_{1} \cdots i_{q}}: 1 \leq i_{j} \leq \ell, 1 \leq j \leq q\right\}$ and $\left\{S_{i_{1} \cdots i_{q}}: 1 \leq i_{j} \leq \ell, 1 \leq j \leq q\right\}$, analogously to (6.9) we have

$$
\limsup _{r \rightarrow 0} \frac{\log m_{x}^{\eta}\left(B^{\pi}(x, r)\right)}{\log r} \leq \frac{\mathbf{E}_{m}\left(g_{q} \mid \mathcal{I}\right)(x)}{\mathbf{E}_{m}\left(\log h_{q} \mid \mathcal{I}\right)(x)},
$$

where

$$
\begin{aligned}
g_{q}:= & \mathbf{I}_{m}\left(\mathcal{P}_{0}^{q-1} \mid \sigma^{-q} \phi^{-1} \mathcal{B}\left(\mathbb{R}^{d}\right)\right)-\mathbf{I}_{m}\left(\mathcal{P}_{0}^{q-1} \mid \phi^{-1} \mathcal{B}\left(\mathbb{R}^{d}\right)\right) \\
& +\mathbf{I}_{m}\left(\mathcal{P}_{0}^{q-1} \mid(\phi, \pi)^{-1} \mathcal{B}\left(\mathbb{R}^{d} \times \mathbb{R}^{k}\right)\right) \\
& -\mathbf{I}_{m}\left(\mathcal{P}_{0}^{q-1} \mid \sigma^{-q}(\phi, \pi)^{-1} \mathcal{B}\left(\mathbb{R}^{d} \times \mathbb{R}^{k}\right)\right)
\end{aligned}
$$

and $h_{q}(x):=\left\|S_{x_{1} \cdots x_{q}}^{\prime}\left(\sigma^{q} x\right)\right\|$ for $x=\left(x_{i}\right)_{i=1}^{\infty}$.

Due to (4.4), we have $\mathbf{E}_{m}\left(g_{q} \mid \mathcal{I}\right)(x)=q \mathbf{E}_{m}(g \mid \mathcal{I})(x)$. It is easily seen that $h_{q}(x)$ is sub-multiplicative in the sense that $h_{p+q}(x) \leq h_{p}(x) h_{q}\left(\sigma^{p} x\right)$. Thus by the Kingman sub-additive ergodic theorem (cf. [63]), we have

$$
\lim _{q \rightarrow \infty} \frac{1}{q} \mathbf{E}_{m}\left(\log h_{q} \mid \mathcal{I}\right)(x)=-\underline{\lambda}(x) \text { for } m \text {-a.e. } x .
$$

Hence letting $q \rightarrow \infty$ in (6.10) we obtain (6.7). This finishes the proof of Theorem 6.2. 
Proof of Theorem 2.6: In Theorem 6.2, we take $T_{i}(x)=\frac{x}{2}$ for all $1 \leq i \leq$ $\ell$ to obtain Theorem 2.6. To see this, we know that the attractor of $\left\{T_{i}\right\}_{i=1}^{\ell}$ is just the singleton $\{0\}$. Hence $\eta$ is the trivial partition $\{\Sigma, \varnothing\}$ of $\Sigma$, and thus we have $m_{x}^{\eta} \equiv m$.

\section{Proofs of Theorem 2.11 and Theorem 2.12}

\subsection{Proof of Theorem 2.11}

Let $\Phi=\left\{S_{i}\right\}_{i=1}^{\ell}$ be the direct product of $k C^{1}$ IFSs $\Phi_{1}, \ldots, \Phi_{k}$, which are defined, respectively, on compact $X_{i} \subset \mathbb{R}^{q_{i}}, i=1, \ldots, k$. For each $i$, let $\Gamma_{i}$ denote the canonical projection with respect to $\Phi_{i}$, and let $\lambda_{i}(x)$ denote the Lyapunov exponent of $\Phi_{i}$ at $x$ provided it exists.

Let $m \in \mathcal{M}_{\sigma}(\Sigma)$. Assume that $\Phi_{1}, \ldots, \Phi_{k}$ are $m$-conformal. Let $\Omega$ denote the collection of all permutations of $\{1, \ldots, k\}$. For $\tau \in \Omega$, we denote

$$
\Lambda_{\tau}:=\left\{x \in \Sigma: \lambda_{i}(x) \text { exists for all } i, \lambda_{\tau(1)}(x) \leq \cdots \leq \lambda_{\tau(k)}(x)\right\} .
$$

Then $m\left(\bigcup_{\tau \in \Omega} \Lambda_{\tau}\right)=1$. Let $\pi$ denote the canonical projection associated with the IFS $\Phi$. In the following we show that the local dimension $d\left(m \circ \pi^{-1}, \pi x\right)$ exists for $m$-a.e. $x \in \Sigma$.

Without loss of generality we only show that $d\left(m \circ \pi^{-1}, \pi x\right)$ exists for $m$ a.e. $x \in \Lambda_{e}$, where $e$ denotes the identity in $\Omega$. Here we may assume $m\left(\Lambda_{e}\right)>0$. For other $\Lambda_{\tau}$ 's, the proof is essentially identical under a change of coordinates.

For $i=1, \ldots, k$, let $\pi_{i}$ denote the canonical projection with respect to $\Phi_{1} \times$ $\cdots \times \Phi_{i}$. It is clear that $\pi=\pi_{k}$. Bear in mind that

$$
\lambda_{1}(x) \leq \cdots \leq \lambda_{k}(x), \quad x \in \Lambda_{e}
$$

For $i=1, \ldots, k$, we use $\left\{m_{x}^{i}\right\}$ to denote the family of conditional measures $\left\{m_{x}^{\eta_{i}}\right\}$ of $m$ associated with the partition

$$
\eta_{i}=\left\{\pi_{i}^{-1}(z): z \in \prod_{t=1}^{i} \mathbb{R}^{q_{t}}\right\} .
$$

For convenience, we use $\left\{m_{x}^{0}\right\}$ to denote the family of conditional measures of $m$ with the trivial partition $\{\Sigma, \varnothing\}$. It is clear that $m_{x}^{0}=m$ for all $x \in \Sigma$.

For $i=1, \ldots, k$, we give a metric $d_{i}$ on $\prod_{t=1}^{i} \mathbb{R}^{q_{t}}$ by

$$
d_{i}\left(\left(z_{1}, \ldots, z_{i}\right),\left(w_{1}, \ldots, w_{i}\right)\right)=\sup _{1 \leq t \leq i}\left|z_{t}-w_{t}\right|_{\mathbb{R}^{q_{t}}}
$$

and define $d=d_{k}$. We claim that for any $x \in \Lambda_{e}$ and $\epsilon>0$,

$$
\eta_{i}(x) \cap \mathcal{P}_{0}^{n}(x) \subset B^{\pi}\left(x, e^{-n\left(\lambda_{i+1}(x)-\epsilon\right)}\right)
$$

when $n$ is large enough. Here $B^{\pi}(x, r)$ is defined as in (3.1). To see the claim, let $x \in \Lambda_{e}$ and $y \in \eta_{i}(x)$. Then $\pi_{i} y=\pi_{i} x$. Thus

$$
d(\pi y, \pi x)=\sup _{1 \leq t \leq k}\left|\Gamma_{t} y-\Gamma_{t} x\right|_{\mathbb{R}^{q_{t}}}=\sup _{i+1 \leq t \leq k}\left|\Gamma_{t} y-\Gamma_{t} x\right|_{\mathbb{R}^{q_{t}}} .
$$


Since $y \in \mathcal{P}_{0}^{n}(x)$ and $\lambda_{i+1}(x) \leq \cdots \leq \lambda_{k}(x)$, by Proposition 5.6, we have

$$
d(\pi y, \pi x) \leq e^{-n\left(\lambda_{i+1}(x)-\epsilon\right)}
$$

when $n$ is large enough, and (7.1) follows.

For $i=0,1, \ldots, k$ and $x \in \Sigma$, denote

$$
h_{i}(x)=\lim _{n \rightarrow \infty} \frac{-\log m_{x}^{i}\left(\mathcal{P}_{0}^{n}(x)\right)}{n+1}
$$

provided that the limit exists. By Proposition 4.14,

$$
h_{i}(x)=h(\sigma, m, x)-h_{\pi_{i}}(\sigma, m, x) \quad \text { for } m \text {-a.e. } x \in \Sigma .
$$

For $i=0,1, \ldots, k-1$ and $x \in \Sigma$, denote

$$
\vartheta_{i}(x)=\liminf _{r \rightarrow 0} \frac{\log m_{x}^{i}\left(B^{\Gamma_{i+1}}(x, r)\right)}{\log r}
$$

By Theorem 6.2 and (7.2), we have

$$
\vartheta_{i}(x)=\frac{h_{\pi_{i+1}}(\sigma, m, x)-h_{\pi_{i}}(\sigma, m, x)}{\lambda_{i+1}(x)}=\frac{h_{i}(x)-h_{i+1}(x)}{\lambda_{i+1}(x)}
$$

for $m$-a.e. $x \in \Sigma$.

For $i=0,1, \ldots, k$ and $x \in \Sigma$, define

$$
\bar{\delta}_{i}(x)=\limsup _{r \rightarrow 0} \frac{\log m_{x}^{i}\left(B^{\pi}(x, r)\right)}{\log r}, \quad \underline{\delta}_{i}(x)=\liminf _{r \rightarrow 0} \frac{\log m_{x}^{i}\left(B^{\pi}(x, r)\right)}{\log r} .
$$

We claim that

(C1) $\bar{\delta}_{k}(x)=\underline{\delta}_{k}(x)=0$ for all $x \in \Sigma$.

(C2) $h_{i}(x)-h_{i+1}(x) \geq \lambda_{i+1}\left(\bar{\delta}_{i}(x)-\bar{\delta}_{i+1}(x)\right)$ for $m$-a.e. $x \in \Lambda_{e}$ and $i=$ $0,1, \ldots, k-1$.

(C3) $\underline{\delta}_{i+1}(x)+\vartheta_{i}(x) \leq \underline{\delta}_{i}(x)$ for $m$-a.e. $x \in \Lambda_{e}$ and $i=0,1, \ldots, k-1$.

It is easy to see that (C1)-(C3) together with (7.2)-(7.3) force that for $m$-a.e. $x \in$ $\Lambda_{e}, \underline{\delta}_{i}(x)=\bar{\delta}_{i}(x)$ (we denote the common value as $\left.\delta_{i}(x)\right)$ for $i=0,1, \ldots, k$ and, furthermore,

$$
d\left(m \circ \pi^{-1}, \pi x\right)=\delta_{0}(x)=\sum_{i=0}^{k-1} \vartheta_{i}(x)=\sum_{i=0}^{k-1} \frac{h_{i}(x)-h_{i+1}(x)}{\lambda_{i+1}(x)} .
$$

which is the desired result in Theorem 2.11.

In what follows we prove $(\mathrm{C} 1)-(\mathrm{C} 3)$.

ProOF OF (C1): Since $\eta_{k}=\left\{\pi^{-1}(z): z \in \prod_{t=1}^{k} \mathbb{R}^{q_{t}}\right\}$, we have

$$
m_{x}^{k}\left(B^{\pi}(x, r)\right)=m_{x}^{k}\left(\eta_{k}(x)\right)=1
$$

for all $x \in \Sigma$. Thus $\bar{\delta}_{k}(x)=\underline{\delta}_{k}(x)=0$ for all $x \in \Sigma$. 
PROOF OF (C2): We give a proof by contradiction, which is modified from $[40, \S 10.2]$. Assume that (C2) is not true. Then there exists $0 \leq i \leq k$ such that

$$
h_{i}(x)-h_{i+1}(x)<\lambda_{i+1}(x)\left(\bar{\delta}_{i}(x)-\bar{\delta}_{i+1}(x)\right)
$$

on a subset of $\Lambda_{e}$ with positive measure. Hence there exist $\alpha>0$ and real numbers $h_{i}, h_{i+1}, \lambda_{i+1}, \bar{\delta}_{i}, \bar{\delta}_{i+1}$ with $\lambda_{i+1}>0$ such that

$$
h_{i}-h_{i+1}<\lambda_{i+1}\left(\bar{\delta}_{i}-\bar{\delta}_{i+1}\right)-\alpha,
$$

and for any $\epsilon>0$, there exists $B_{\epsilon} \subset \Lambda_{e}$ with $m\left(B_{\epsilon}\right)>0$ so that for $x \in B_{\epsilon}$,

$$
\left|h_{i}(x)-h_{i}\right|<\frac{\epsilon}{2}, \quad\left|h_{i+1}(x)-h_{i+1}\right|<\frac{\epsilon}{2}, \quad\left|\lambda_{i+1}(x)-\lambda_{i+1}\right|<\frac{\epsilon}{2},
$$

and

$$
\left|\bar{\delta}_{i}(x)-\bar{\delta}_{i}\right|<\frac{\epsilon}{2}, \quad\left|\bar{\delta}_{i+1}(x)-\bar{\delta}_{i+1}\right|<\frac{\epsilon}{2} .
$$

Fix $\epsilon>0$. There exists $n_{0}: B_{\epsilon} \rightarrow \mathbb{N}$ such that for $m$-a.e. $x \in B_{\epsilon}$ and $n>n_{0}(x)$, we have
(1) $\frac{\log m_{x}^{i+1}\left(B^{\pi}\left(x, e^{-n\left(\lambda_{i+1}-2 \epsilon\right)}\right)\right)}{-n\left(\lambda_{i+1}-2 \epsilon\right)} \leq \bar{\delta}_{i+1}+\epsilon$;
(2) $-\frac{1}{n} \log m_{x}^{i+1}\left(\mathcal{P}_{0}^{n}(x)\right) \geq h_{i+1}-\epsilon \quad$ (by (7.2));
(3) $\eta_{i}(x) \cap \mathcal{P}_{0}^{n}(x) \subset B^{\pi}\left(x, e^{-n\left(\lambda_{i+1}-2 \epsilon\right)}\right) \quad$ (by (7.1));
(4) $-\frac{1}{n} \log m_{x}^{i}\left(\mathcal{P}_{0}^{n}(x)\right) \leq h_{i}+\epsilon \quad$ (by (7.2)).

Take $N_{0}$ such that

$$
\Delta:=\left\{x \in B_{\epsilon}: n_{0}(x) \leq N_{0}\right\}
$$

has positive measure. By Lemma 3.3 and Lemma 3.10, there exist $c>0$ and $\Delta^{\prime} \subset \Delta$ with $m\left(\Delta^{\prime}\right)>0$ such that for $x \in \Delta^{\prime}$, there exists $n=n(x) \geq N_{0}$ such that

(5) $\frac{m_{x}^{i+1}(L \cap \Delta)}{m_{x}^{i+1}(L)} \geq c \quad$ where $L:=B^{\pi}\left(x, e^{-n\left(\lambda_{i+1}-2 \epsilon\right)}\right)$;

(6) $\frac{\log m_{x}^{i}\left(B^{\pi}\left(x, 2 e^{-n\left(\lambda_{i+1}-2 \epsilon\right)}\right)\right)}{-n\left(\lambda_{i+1}-2 \epsilon\right)}>\bar{\delta}_{i}-\epsilon$;

(7) $\frac{\log (1 / c)}{n}<\epsilon$.

Take $x \in \Delta^{\prime}$ such that (1)-(7) are satisfied with $n=n(x)$. Denote $C=\eta_{i+1}(x)$ and $C^{\prime}=\eta_{i}(x)$. Then by (5) and (1),

$$
m_{x}^{i+1}(L \cap \Delta) \geq c m_{x}^{i+1}(L) \geq c e^{-n\left(\lambda_{i+1}-2 \epsilon\right)\left(\bar{\delta}_{i+1}+\epsilon\right)} .
$$

But for each $y \in L \cap \Delta$, we have by (2), $m_{y}^{i+1}\left(\mathcal{P}_{0}^{n}(y)\right) \leq e^{-n\left(h_{i+1}-\epsilon\right)}$. It follows that the number of distinct $\mathcal{P}_{0}^{n}$-atoms intersecting $C \cap L \cap \Delta$ is larger than

$$
m_{x}^{i+1}(L \cap \Delta) e^{n\left(h_{i+1}-\epsilon\right)} .
$$


However, each such $\mathcal{P}_{0}^{n}$-atom, say $\mathcal{P}_{0}^{n}(y)$, intersects $C^{\prime} \cap L \cap \Delta$, and this together with (3) guarantees that $C^{\prime} \cap \mathcal{P}_{0}^{n}(y)$ is contained in $C^{\prime} \cap B^{\pi}\left(x, 2 e^{-n\left(\lambda_{i+1}-2 \epsilon\right)}\right)$. To see this, let $z \in \mathcal{P}_{0}^{n}(y) \cap C^{\prime} \cap L \cap \Delta$. Since $z \in \Delta$, we have $d(\pi z, \pi x) \leq$ $e^{-n\left(\lambda_{i+1}-2 \epsilon\right)}$. Thus

$$
\begin{aligned}
\mathcal{C}^{\prime} \cap \mathcal{P}_{0}^{n}(y)=\eta_{i}(z) \cap \mathcal{P}_{0}^{n}(z) & \subset B^{\pi}\left(x, e^{-n\left(\lambda_{i+1}-2 \epsilon\right)}\right) \\
& \subset B^{\pi}\left(x, 2 e^{-n\left(\lambda_{i+1}-2 \epsilon\right)}\right) .
\end{aligned}
$$

Meanwhile by (4), $m_{x}^{i}\left(\mathcal{P}_{0}^{n}(y)\right) \geq e^{-n\left(h_{i}+\epsilon\right)}$ (for $w \in \mathcal{P}_{0}^{n}(y) \cap C^{\prime} \cap L$, we have $\eta_{i}(x)=\eta_{i}(w)$ and thus $\left.m_{x}^{i}\left(\mathcal{P}_{0}^{n}(y)\right)=m_{w}^{i}\left(\mathcal{P}_{0}^{n}(w)\right)\right)$. Hence we have

$$
\begin{aligned}
& m_{x}^{i}\left(B^{\pi}\left(x, 2 e^{-n\left(\lambda_{i+1}-2 \epsilon\right)}\right)\right) \\
& \quad \geq \#\left\{\mathcal{P}_{0}^{n} \text {-atoms intersecting } C^{\prime} \cap L \cap \Delta\right\} \cdot e^{-n\left(h_{i}+\epsilon\right)} \\
& \quad \geq m_{x}^{i+1}(L \cap \Delta) e^{n\left(h_{i+1}-\epsilon\right)} e^{-n\left(h_{i}+\epsilon\right)} \\
& \quad \geq c e^{-n\left(\lambda_{i+1}-2 \epsilon\right)\left(\bar{\delta}_{i+1}+\epsilon\right)} e^{n\left(h_{i+1}-\epsilon\right)} e^{-n\left(h_{i}+\epsilon\right)} .
\end{aligned}
$$

Comparing this with (6), we have

$$
\begin{aligned}
& \left(\lambda_{i+1}-2 \epsilon\right)\left(\bar{\delta}_{i}-\epsilon\right) \\
& \quad \leq\left(\lambda_{i+1}-2 \epsilon\right)\left(\bar{\delta}_{i+1}+\epsilon\right)\left(\lambda_{i}-2 \epsilon\right)+\frac{\log (1 / c)}{n}+h_{i}-h_{i+1}+2 \epsilon \\
& \quad \leq\left(\lambda_{i+1}-2 \epsilon\right)\left(\bar{\delta}_{i+1}+\epsilon\right)\left(\lambda_{i}-2 \epsilon\right)+h_{i}-h_{i+1}+3 \epsilon .
\end{aligned}
$$

Taking $\epsilon \rightarrow 0$ yields $h_{i}-h_{i+1} \geq \lambda_{i+1}\left(\bar{\delta}_{i}-\bar{\delta}_{i+1}\right)$, which leads to a contradiction with (7.5).

ProOF OF (C3): Here we give a proof by contradiction, adopting an idea from the proof of [40, lemma 11.3.1]. Assume that (C3) is not true. Then there exists $0 \leq i \leq k-1$ such that $\underline{\delta}_{i+1}(x)+\vartheta_{i}(x)>\underline{\delta}_{i}(x)$ on a subset of $\Lambda_{e}$ with positive measure. Hence there exists $\beta>0$ and real numbers $\underline{\delta}_{i}, \underline{\delta}_{i+1}, \lambda_{i}$ such that

$$
\underline{\delta}_{i+1}+\vartheta_{i}>\underline{\delta}_{i}+\beta,
$$

and for any $\epsilon>0$, there exists $A_{\epsilon} \subset \Lambda_{e}$ with $m\left(A_{\epsilon}\right)>0$ so that for $x \in A_{\epsilon}$,

$$
\left|\underline{\delta}_{i}(x)-\underline{\delta}_{i}\right|<\frac{\epsilon}{2}, \quad\left|\underline{\delta}_{i+1}(x)-\underline{\delta}_{i+1}\right|<\frac{\epsilon}{2}, \quad\left|\vartheta_{i}(x)-\vartheta_{i}\right|<\frac{\epsilon}{2} .
$$

Let $0<\epsilon<\frac{\beta}{4}$. Find $N_{1}$ and a set $A_{\epsilon}^{\prime} \subset A_{\epsilon}$ with $m\left(A_{\epsilon}^{\prime}\right)>0$ such that for $x \in A_{\epsilon}^{\prime}$ and $n>N_{1}$,

$$
m_{x}^{i+1}\left(B^{\pi}\left(x, 2 e^{-n}\right)\right) \leq e^{-n\left(\underline{\delta}_{i+1}-\epsilon\right)} .
$$

By Lemma 3.3 and Lemma 3.10, we can find $c>0$ and $A_{\epsilon}^{\prime \prime} \subset A_{\epsilon}^{\prime}$ with $m\left(A_{\epsilon}^{\prime \prime}\right)>0$ and $N_{2}$ such that for all $x \in A_{\epsilon}^{\prime \prime}$ and $n \geq N_{2}$,

$$
\frac{m_{x}^{i}\left(A_{\epsilon}^{\prime} \cap B^{\pi}\left(x, e^{-n}\right)\right)}{m_{x}^{i}\left(B^{\pi}\left(x, e^{-n}\right)\right)}>c .
$$


For $x \in A_{\epsilon}^{\prime \prime}$ and $n \geq N_{2}$, we have

$$
\begin{aligned}
m_{x}^{i}\left(B^{\pi}\left(x, e^{-n}\right)\right) & \leq c^{-1} m_{x}^{i}\left(A_{\epsilon}^{\prime} \cap B^{\pi}\left(x, e^{-n}\right)\right) \\
& =c^{-1} \int m_{y}^{i+1}\left(A_{\epsilon}^{\prime} \cap B^{\pi}\left(x, e^{-n}\right)\right) d m_{x}^{i}(y) \\
& =c^{-1} \int_{B^{\Gamma_{i+1}}\left(x, e^{-n}\right)} m_{y}^{i+1}\left(A_{\epsilon}^{\prime} \cap B^{\pi}\left(x, e^{-n}\right)\right) d m_{x}^{i}(y) .
\end{aligned}
$$

Let $y \in \eta_{i}(x)$ be such that $\eta_{i+1}(y) \cap A_{\epsilon}^{\prime} \cap B^{\pi}\left(x, e^{-n}\right) \neq \varnothing$. Then there exists $w \in A_{\epsilon}^{\prime} \cap B^{\pi}\left(x, e^{-n}\right)$ such that $\pi_{i+1} y=\pi_{i+1} w$. Hence $A_{\epsilon}^{\prime} \cap B^{\pi}\left(x, e^{-n}\right) \subset$ $B^{\pi}\left(w, 2 e^{-n}\right)$ and by (7.8)

$$
\begin{aligned}
m_{y}^{i+1}\left(A_{\epsilon}^{\prime} \cap B^{\pi}\left(w, e^{-n}\right)\right) & =m_{w}^{i+1}\left(A_{\epsilon}^{\prime} \cap B^{\pi}\left(w, e^{-n}\right)\right) \\
& \leq m_{w}^{i+1}\left(B^{\pi}\left(w, 2 e^{-n}\right)\right) \\
& \leq e^{-n\left(\underline{\delta}_{i+1}-\epsilon\right)} .
\end{aligned}
$$

Combining this with (7.9), we have

$$
m_{x}^{i}\left(B^{\pi}\left(x, e^{-n}\right)\right) \leq c^{-1} e^{-n\left(\underline{\delta}_{i+1}-\sigma\right)} m_{x}^{i}\left(B^{\Gamma_{i+1}}\left(x, e^{-n}\right)\right), \quad x \in A_{\epsilon}^{\prime \prime}, n \geq N_{2} .
$$

Letting $n \rightarrow \infty$, we obtain $\underline{\delta}_{i}(x) \geq \underline{\delta}_{i+1}-\epsilon+\vartheta_{i}(x)$ for $x \in A_{\epsilon}^{\prime \prime}$. Combining this with (7.7) yields

$$
\underline{\delta}_{i} \geq \underline{\delta}_{i+1}+\vartheta_{i}-4 \epsilon \geq \underline{\delta}_{i+1}+\vartheta_{i}-\beta
$$

which contradicts (7.6).

\subsection{Proof of Theorem $\mathbf{2 . 1 2}$}

DEFINITIOn 7.1 A real square matrix $A$ is called asymptotically similar if all the (complex) eigenvalues of $A$ are equal in modulus. Correspondingly, a linear transformation $T$ on a finite-dimensional vector space $V$ is called asymptotically similar if its representation matrix (associated with some basis of $V$ ) is asymptotically similar.

LEMMA 7.2 Let $\left(A_{1}, \ldots, A_{\ell}\right)$ be an $\ell$-tuple of commuting linear transformations on $\mathbb{R}^{d}$. Then there are subspaces $V_{1}, \ldots, V_{k}$ of $\mathbb{R}^{d}$ such that

(i) $\mathbb{R}^{d}=V_{1} \oplus \cdots \oplus V_{k}$;

(ii) $V_{i}$ is $A_{j}$-invariant for $1 \leq i \leq k$ and $1 \leq j \leq \ell$;

(iii) the restriction of $A_{j}$ on $V_{i}$ is asymptotically similar for $1 \leq i \leq k$ and $1 \leq j \leq \ell$.

Proof: For brevity, we only prove the lemma in the case $\ell=2$. The reader will see that the idea works for all cases.

Let $S, T$ be two commuting linear transformations on $\mathbb{R}^{d}$. Let $f$ denote the real minimal polynomial of $S$. Suppose $f=f_{1}^{t_{1}} \cdots f_{p}^{t_{p}}$ is the decomposition of $f$ into powers of distinct, real irreducible monic factors $f_{i}$. Let $W_{i}$ denote the null space 
of $\left[f_{i}(S)\right]^{t_{i}}, i=1, \ldots, p$. Then $W_{i}$ 's are $S$-invariant and $\mathbb{R}^{d}=W_{1} \oplus \cdots \oplus W_{p}$ (cf. [62, theorem 7.3]). Moreover, $S_{W_{i}}$, the restriction of $S$ to $W_{i}$, is asymptotically similar.

Since $S T=T S, W_{i}$ is also $T$-invariant for each $i$. But $T_{W_{i}}$ may be not asymptotically similar. However, as above, for each $i$, we can decompose $W_{i}$ into $W_{i}=W_{i, 1} \oplus \cdots \oplus W_{i, u_{i}}$ such that $W_{i, j}$ are the null spaces corresponding to some factors of the minimal polynomial of $T_{W_{i}}$. Again, $W_{i, j}$ is $T_{W_{i}}$-invariant and $S_{W_{i}}$-invariant. Furthermore, $T_{W_{i, j}}$ and $S_{W_{i, j}}$ are asymptotically similar. Hence $\mathbb{R}^{d}=\bigoplus_{i, j} W_{i, j}$ is the desired decomposition for $S$ and $T$.

PRoOF OF THEOREM 2.12: Let $\left\{S_{i}\right\}_{i=1}^{\ell}$ be the IFS given in the theorem. Then there is a nonsingular linear transformation $Q$ on $\mathbb{R}^{d}$ such that $\left\{Q S_{i} Q^{-1}\right\}_{i=1}^{\ell}$ is the direct product of $k$ asymptotically conformal IFS by Lemma 7.2. Hence the desired result follows from Theorem 2.11.

\section{A Variational Principle about Dimensions of Self-Conformal Sets}

In this section, we assume that $K$ is the attractor of a $C^{1}$ weakly conformal IFS $\Phi=\left\{S_{i}\right\}_{i=1}^{\ell}$ on a compact set $X \subset \mathbb{R}^{d}$. The main result of this section is the following variational principle (i.e., Theorem 2.13):

THEOREM 8.1 Under the above setting, we have

$$
\begin{aligned}
\operatorname{dim}_{H} K & =\operatorname{dim}_{B} K \\
& =\sup \left\{\operatorname{dim}_{H} \mu: \mu=m \circ \pi^{-1}, m \in \mathcal{M}_{\sigma}(\Sigma), m \text { is ergodic }\right\} \\
& =\max \left\{\operatorname{dim}_{H} \mu: \mu=m \circ \pi^{-1}, m \in \mathcal{M}_{\sigma}(\Sigma)\right\} \\
& =\sup \left\{\frac{h_{\pi}(\sigma, m)}{\int \lambda d m}: m \in \mathcal{M}_{\sigma}(\Sigma)\right\} .
\end{aligned}
$$

PROOF: Without loss of generality we assume that $\overline{\operatorname{dim}}_{B}(K)>0$, where $\overline{\operatorname{dim}}_{B}$ denotes the upper box-counting dimension (cf. [13]). Let

$$
0<t_{3}<t_{2}<t_{1}<\overline{\operatorname{dim}}_{B}(K) .
$$

We first prove that there is an ergodic measure $m \in \mathcal{M}_{\sigma}(\Sigma)$ such that $\operatorname{dim}_{H} m \circ$ $\pi^{-1} \geq t_{3}$. To achieve this, let $\alpha=t_{2} / t_{3}-1$, and let $r_{0}$ be given as in Corollary 5.5. Since $\operatorname{dim}_{B}(K)>t_{1}$, for any $0<\epsilon<r_{0}$, there exist $r \in(0, \epsilon)$ and integer $N \geq r^{-t_{1}}$ such that there are disjoint closed balls $B\left(z_{i}, r\right), i=1, \ldots, N$, with centers $z_{i} \in K$. By Corollary 5.5, we can find words $w_{i} \in \Sigma^{*}, i=1, \ldots, N$, such that $S_{w_{i}}(K) \subset B\left(z_{i}, r\right)$ and

$$
\left|S_{w_{i}}(x)-S_{w_{i}}(y)\right| \geq r^{1+\alpha}|x-y|, \quad x, y \in K .
$$


This implies $r^{1+\alpha} \operatorname{diam}(K) \leq \operatorname{diam}\left(S_{w_{i}}(K)\right) \leq 2 r$. According to this fact and (5.6), there exist two positive constants $A, B$ (independent of $r$ ) such that

$$
B \log \left(\frac{1}{r}\right) \leq\left|w_{i}\right| \leq A \log \left(\frac{1}{r}\right) \quad \text { for all } 1 \leq i \leq N .
$$

Hence by the pigeonhole principle, there is a subset $\mathcal{J}$ of $\{1, \ldots, N\}$ with cardinality

$$
\# \mathcal{J} \geq \frac{N}{(A-B) \log \left(\frac{1}{r}\right)+1} \geq \frac{r^{-t_{1}}}{(A-B) \log \left(\frac{1}{r}\right)+1} \geq r^{-t_{2}}
$$

such that the words $w_{i}(i \in \mathcal{J})$ have the same length, say $n$.

Now we adopt an argument from the proof of [12, theorem 4]. Let

$$
\delta=\min \left\{d\left(B\left(z_{i}, r\right), B\left(z_{j}, r\right)\right): i, j \in \mathcal{J}, i \neq j\right\} .
$$

For any positive integer $q$ and distinct sequences $i_{1}, \ldots, i_{q}$ and $j_{1}, \ldots, j_{q}$ taking values in $\mathcal{J}$, let $k$ be the least integer such that $i_{k} \neq j_{k}$. Applying (8.5) $(k-1)$ times, we have

$$
\begin{aligned}
& d\left(S_{w_{i_{1}}} \circ \cdots \circ S_{w_{i_{q}}}(K), S_{w_{j_{1}}} \circ \cdots \circ S_{w_{j_{q}}}(K)\right) \geq \\
& \quad r^{(1+\alpha)(k-1)} d\left(B\left(z_{i_{k}}, r\right), B\left(z_{j_{k}}, r\right)\right) \geq r^{q(1+\alpha)} \delta .
\end{aligned}
$$

Define a measure $\eta$ on the class of finite unions of sets $S_{w_{i_{1}}} \circ \cdots \circ S_{w_{i_{q}}}(K)$ by letting $\eta\left(S_{w_{i_{1}}} \circ \cdots \circ S_{w_{i_{q}}}(K)\right)=(\# \mathcal{J})^{-q}$. This extends to a measure $\eta$ on the $\sigma$-algebra generated by these sets. Let $U$ be any subset of $K$ with $\operatorname{diam}(U)<\delta$ and let $q$ be the least integer such that

$$
r^{(q+1)(1+\alpha)} \delta \leq \operatorname{diam}(U)<r^{q(1+\alpha)} \delta
$$

Then $U$ intersects at most one set $S_{w_{i_{1}}} \circ \cdots \circ S_{w_{i_{q}}}(K)$, hence

$$
\begin{aligned}
\eta(U) \leq(\# \mathcal{J})^{-q} \leq r^{t_{2}} q & \leq r^{-t_{2}} \delta^{-\frac{t_{2}}{1+\alpha}} \operatorname{diam}(U)^{\frac{t_{2}}{1+\alpha}} \\
& =r^{-t_{2}} \delta^{-t_{3}} \operatorname{diam}(U)^{t_{3}} .
\end{aligned}
$$

This implies $\operatorname{dim}_{H} \eta \geq t_{3}$.

We point out that the measure $\eta$ constructed as above is, indeed, the projection of a $\sigma^{n}$-invariant and ergodic measure $v$ under $\pi$. Actually, $v$ is the unique measure on $\Sigma$ satisfying

$$
v\left(\left[w_{i_{1}} \cdots w_{i_{q}}\right]\right)=(\# \mathcal{J})^{-q}, \quad q \in \mathbb{N}, i_{1}, \ldots, i_{q} \in \mathcal{J} .
$$

Applying Theorem 2.8 to the IFS $\left\{S_{w_{i}}: i \in \mathcal{J}\right\}$, we have

$$
\operatorname{dim}_{H} \eta=\operatorname{dim}_{H} v \circ \pi^{-1}=\frac{h_{\pi}\left(\sigma^{n}, v\right)}{-\int \log \left\|S_{x_{1} \cdots x_{n}}^{\prime}\left(\pi \sigma^{n} x\right)\right\| d v} .
$$


Take $m=\frac{1}{n} \sum_{i=0}^{n-1} v \circ \sigma^{-i}$. Then $m$ is $\sigma$-invariant and ergodic. Applying Theorem 2.8 and Proposition 4.3, we have

$$
\begin{aligned}
\operatorname{dim}_{H} m \circ \pi^{-1}=\frac{h_{\pi}(\sigma, m)}{-\int \log \left\|S_{x_{1}}^{\prime}(\pi \sigma x)\right\| d m} & =\frac{h_{\pi}\left(\sigma^{n}, v\right)}{-\int \log \left\|S_{x_{1} \cdots x_{n}}^{\prime}\left(\pi \sigma^{n} x\right)\right\| d v} \\
& =\operatorname{dim}_{H} \eta \geq t_{3} .
\end{aligned}
$$

Since $t_{3}<\overline{\operatorname{dim}}_{B} K$ is arbitrarily given, we obtain (8.1) and (8.2).

To show (8.3), let $\left(m_{i}\right)$ be a sequence of measures in $\mathcal{M}_{\sigma}(\Sigma)$ with

$$
\lim _{i \rightarrow \infty} \operatorname{dim}_{H} m_{i} \circ \pi^{-1}=\operatorname{dim}_{H} K .
$$

Take a sequence of positive numbers $\left(a_{i}\right)$ such that $\sum_{i=1}^{\infty} a_{i}=1$. Then $m=$ $\sum_{i=1}^{\infty} a_{i} m_{i}$ is an element in $\mathcal{M}_{\sigma}(\Sigma)$ with

$$
\operatorname{dim}_{H} m \circ \pi^{-1}=\sup _{i} \operatorname{dim}_{H} m_{i} \circ \pi^{-1}=\operatorname{dim}_{H} K .
$$

To show (8.4), according to (8.2), it suffices to show that

$$
\operatorname{dim}_{H} m \circ \pi^{-1} \geq \frac{h_{\pi}(\sigma, m)}{-\int \log \left\|S_{x_{1}}^{\prime}(\pi \sigma x)\right\| d m(x)}, \quad m \in \mathcal{M}_{\sigma}(\Sigma) .
$$

Fix $m$ and let $\mu=m \circ \pi^{-1}$. Denote by $\Lambda$ the right-hand side of (8.6). By Theorem $2.8, d(\mu, z)$ exists for $\mu$-a.e. $z \in \mathbb{R}^{d}$. Hence to show (8.6), we only need to show that for any $\epsilon>0$, there is a Borel set $E \subset \mathbb{R}^{d}$ such that $\mu(E)>0$ and $d(\mu, z) \geq \Lambda-\epsilon$ for $z \in E$. Assume this is false. Then $d(\mu, z)<\Lambda-\epsilon$ for $\mu$-a.e. $z \in \mathbb{R}^{d}$. Thus by Theorem 2.8 again, we have

$$
h_{\pi}(\sigma, m, x)<\lambda(x)(\Lambda-\epsilon) \text { for } m \text {-a.e. } x \in \Sigma \text {. }
$$

Taking integration with respect to $m$ on both sides yields

$$
h_{\pi}(\sigma, m)<(\Lambda-\epsilon) \int \lambda d m
$$

which leads to a contradiction.

Remark 8.2. Assume that $\left\{S_{i}\right\}_{i=1}^{\ell}$ is a weakly conformal IFS that satisfies the AWSC (see Definition 2.14). Then the supremum in (8.2) and (8.4) can be attained by ergodic measures. To see this, by Proposition 4.20, the map $m \mapsto h_{\pi}(\sigma, m)$ is upper semicontinuous on $\mathcal{M}_{\sigma}(\Sigma)$; hence the supremum in (8.4) is attained at some member, say $m_{0}$, in $\mathcal{M}_{\sigma}(\Sigma)$. Let $m_{0}=\int \nu d \mathbb{P}(v)$ be the ergodic decomposition of $m_{0}$. By Theorem 2.2(ii), we have

$$
\operatorname{dim}_{H} K=\frac{h_{\pi}\left(\sigma, m_{0}\right)}{\int \lambda d m_{0}}=\frac{\int h_{\pi}(\sigma, v) d \mathbb{P}(v)}{\iint \lambda d \nu d \mathbb{P}(v)} .
$$

Since $h_{\pi}(\sigma, v) / \int \lambda d v \leq \operatorname{dim}_{H} K$ for each $v$, the above equality implies that $h_{\pi}(\sigma, v) / \int \lambda d v=\operatorname{dim}_{H} K$ for $\mathbb{P}$-a.e. $v$. Hence the supremum in (8.4) can be attained at some ergodic measure, so do the supremum in (8.2). 
D.-J. FENG AND H. HU

\section{Proof of Theorem 2.15}

We first present some lemmas.

LEMMA 9.1 Let $\left\{S_{i}\right\}_{i=1}^{\ell}$ be an IFS with attractor $K$. For $n \in \mathbb{N}$, write $\Sigma_{n}=$ $\{1, \ldots, \ell\}^{n}$ and denote

$$
N_{n}=\#\left\{S_{u}: u \in \Sigma_{n}\right\} .
$$

Then

(1) $\sup \left\{h_{\pi}(\sigma, m): m \in \mathcal{M}_{\sigma}(\Sigma)\right\} \leq \log N_{n} / n$.

(2) Let $t_{n}=\sup _{x \in \mathbb{R}^{d}} \#\left\{S_{u}: u \in \Sigma_{n}, x \in S_{u}(K)\right\}$. Then

$$
\sup \left\{h_{\pi}(\sigma, m): m \in \mathcal{M}_{\sigma}(\Sigma), \text { is ergodic }\right\} \geq \frac{\log N_{n}-\log t_{n}}{n} .
$$

Proof: We first show (i). Let $n \in \mathbb{N}$ and $m \in \mathcal{M}_{\sigma}(\Sigma)$. By the definition of $N_{n}$, we can construct a subset $\Omega$ of $\Sigma_{n}$ with $\# \Omega=N_{n}$ such that for any $u \in \Sigma_{n}$, there exists $w \in \Omega$ so that $S_{u}=S_{w}$. Hence there is a map $g: \Sigma_{n} \rightarrow \Omega$ such that $S_{u}=S_{g(u)}$ for each $u \in \Sigma_{n}$. Let $\left(\Omega^{\mathbb{N}}, T\right)$ denote the one-sided full shift over $\Omega$. Define $G: \Sigma \rightarrow \Omega^{\mathbb{N}}$ by

$$
G\left(\left(x_{i}\right)_{i=0}^{\infty}\right)=\left(w_{j}\right)_{j=1}^{\infty} \quad\left(\left(x_{i}\right)_{i=1}^{\infty} \in \Sigma\right),
$$

where $w_{j}=g\left(x_{(j-1) n+1} x_{(j-1) n+2} \cdots x_{j n}\right)$. Let $\tilde{\pi}: \Omega^{\mathbb{N}} \rightarrow \mathbb{R}^{d}$ denote the canonical projection with respect to the IFS $\left\{S_{u}: u \in \Omega\right\}$. By Lemma 4.23(ii), we then have

$$
h_{\pi}\left(\sigma^{n}, m\right)=h_{\tilde{\pi}}\left(T, m \circ G^{-1}\right) \leq \log (\# \Omega)=\log N_{n} .
$$

It follows that $h_{\pi}(\sigma, m) \leq \log N_{n} / n$. This proves (i).

To show (ii), let $v$ be the Bernoulli measure on $\Omega^{\mathbb{N}}$ with probability weight $\left(1 / N_{n}, \ldots, 1 / N_{n}\right)$. Then $v$ can be viewed as a $\sigma^{n}$-invariant measure on $\Sigma$. By Lemma 4.23(ii), we have $h_{\pi}\left(\sigma^{n}, v\right)=h_{\tilde{\pi}}(T, v)$. Note that for $x \in \mathbb{R}^{d}$, there are at most $t_{n}$ words $u$ in $\Omega$ such that $x \in S_{u}(K)$. By Corollary 4.22, we have

$$
h_{\tilde{\pi}}(T, v) \geq h(T, v)-\log t_{n}=\log N_{n}-\log t_{n} .
$$

Let $\mu=\frac{1}{n} \sum_{i=0}^{n-1} \nu \circ \sigma^{-i}$. Then $\mu$ is $\sigma$-invariant and ergodic; furthermore,

$$
h_{\pi}(\sigma, \mu)=\frac{1}{n} h_{\pi}\left(\sigma^{n}, v\right)=\frac{1}{n} h_{\tilde{\pi}}(T, v) \geq \frac{\log N_{n}-\log t_{n}}{n},
$$

as desired.

LEMMA 9.2 Let $\Phi=\left\{S_{i}\right\}_{i=1}^{\ell}$ be an affine IFS on $\mathbb{R}^{d}$ given by

$$
S_{i}\left(x_{1}, \ldots, x_{d}\right)=\left(\rho_{1} x_{1}, \cdots, \rho_{d} x_{d}\right)+\left(a_{i, 1}, \ldots, a_{i, d}\right),
$$

where $1>\rho_{1}>\cdots>\rho_{d}>0$ and $a_{i, j} \in \mathbb{R}$. Let $K$ denote the attractor of $\Phi$, and write $\lambda_{j}=\log \left(1 / \rho_{j}\right)$ for $j=1, \ldots, d$ and $\lambda_{d+1}=\infty$. View $\Phi$ as the direct 
product of $\Phi_{1}, \ldots, \Phi_{d}$, where $\Phi_{j}=\left\{S_{i, j}\left(x_{j}\right)=\rho_{j} x_{j}+a_{i, j}\right\}_{i=1}^{\ell}$. Let $\pi_{j}$ denote the canonical projection with respect to the IFS $\Phi_{1} \times \cdots \times \Phi_{j}$. Then we have

$$
\begin{aligned}
\sum_{j=1}^{d}\left(\frac{1}{\lambda_{j}}-\frac{1}{\lambda_{j+1}}\right) H_{j} \leq \underline{\operatorname{dim}}_{B}(K) & \leq \overline{\operatorname{dim}}_{B}(K) \\
& \leq \sum_{j=1}^{d}\left(\frac{1}{\lambda_{j}}-\frac{1}{\lambda_{j+1}}\right) \tilde{H}_{j},
\end{aligned}
$$

with $H_{j}=\sup \left\{h_{\pi_{j}}(\sigma, m): m \in \mathcal{M}_{\sigma}(\Sigma)\right\}$ and

$$
\tilde{H}_{j}=\lim _{n \rightarrow \infty} \frac{\log \#\left\{S_{u}^{(j)}: u \in \Sigma_{n}\right\}}{n},
$$

where $\left\{S_{i}^{(j)}\right\}_{i=1}^{\ell}$ is the IFS $\Phi_{1} \times \cdots \times \Phi_{j}$ on $\mathbb{R}^{j}$.

PROOF: Without loss of generality we assume that

$$
S_{i}\left([0,1]^{d}\right) \subset[0,1]^{d}, \quad i=1, \ldots, \ell .
$$

For $n \in \mathbb{N}$, we write

$$
N_{n}^{(j)}=\#\left\{S_{u}^{(j)}: u \in \Sigma_{n}\right\}, \quad j=1, \ldots, d,
$$

and

$$
q_{d}(n)=n, \quad q_{j}(n)=\left[\left(\frac{\log \rho_{d}}{\log \rho_{j}}-\frac{\log \rho_{d}}{\log \rho_{j+1}}\right) n\right] \quad \text { for } 1 \leq j \leq d-1,
$$

where $[x]$ denotes the integral part of $x$.

Construct $\Omega_{n, j} \subset \Sigma_{q_{j}(n)}, j=1, \ldots, d$, such that $\# \Omega_{n, j}=N_{q_{j}(n)}^{(j)}$ and for each $u \in \Sigma_{q_{j}(n)}$, there is $w \in \Omega_{n, j}$ so that $S_{u}^{(j)}=S_{w}^{(j)}$. Then the family of the rectangles

$$
\prod_{j=1}^{d} S_{w_{d} w_{d-1} \cdots w_{j}, j}([0,1]), \quad w_{1} \in \Omega_{n, 1}, \ldots, w_{d} \in \Omega_{n, d},
$$

is a cover of $K$. To see this, let $u_{j} \in \Sigma_{q_{j}(n)}, j=1, \ldots, d$. Then we can find $w_{j} \in \Omega_{n, j}, j=1, \ldots, d$, such that $S_{u_{j}}^{(j)}=S_{w_{j}}^{(j)}$. Hence

$$
\begin{aligned}
S_{u_{d} u_{d-1} \cdots u_{1}}(K) \subset & S_{u_{d} u_{d-1} \cdots u_{1}}\left([0,1]^{d}\right) \subset \prod_{j=1}^{d} S_{u_{d} u_{d-1} \cdots u_{1}, j}([0,1]) \\
& \subset \prod_{j=1}^{d} S_{u_{d} u_{d-1} \cdots u_{j}, j}([0,1])=\prod_{j=1}^{d} S_{w_{d} w_{d-1} \cdots w_{j}, j}([0,1]) .
\end{aligned}
$$


It follows that the family of rectangles in (9.2) covers $K$. One can check that each rectangle in (9.2) is an almost $\left(\rho_{d}\right)^{n}$-cube. Hence by the definition of box-counting dimension, we have

$$
\begin{aligned}
\overline{\operatorname{dim}}_{B} K \leq \limsup _{n \rightarrow \infty} \frac{\prod_{j=1}^{d} \# \Omega_{n, j}}{-\log \left(\rho_{d}\right)^{n}} & =\limsup _{n \rightarrow \infty} \frac{\prod_{j=1}^{d} N_{q_{j}(n)}^{(j)}}{-\log \left(\rho_{d}\right)^{n}} \\
& =\sum_{j=1}^{d}\left(\frac{1}{\lambda_{j}}-\frac{1}{\lambda_{j+1}}\right) \tilde{H}_{j} .
\end{aligned}
$$

This proves one part of (9.1).

To see the other part of $(9.1)$, for $j=1, \ldots, d$, let $\mathcal{Q}_{j}$ denote the collection $\left\{[0,1)^{j}+\alpha: \alpha \in \mathbb{Z}^{j}\right\}$, and define

$$
M_{n}^{(j)}=\#\left\{Q \in \mathcal{Q}_{j}: \operatorname{diag}\left(\rho_{1}^{n}, \ldots, \rho_{j}^{n}\right) Q \cap K_{j} \neq \varnothing\right\},
$$

where $K_{j}$ denotes the attractor of $\Phi_{1} \times \cdots \times \Phi_{j}$. Then by Proposition 4.18(ii), we have $H_{j}=\lim _{n \rightarrow \infty} \log M_{n}^{(j)} / n$. We claim that for $n \in \mathbb{N}$, there exists a subset $\bar{\Omega}_{n, j} \subset \Sigma_{n}$ with cardinality $\geq 7^{-j} M_{n}^{(j)}$ such that

$$
S_{w}^{(j)}\left([0,1]^{j}\right) \cap S_{w^{\prime}}^{(j)}\left([0,1]^{j}\right)=\varnothing \quad \text { for all } w, w^{\prime} \in \bar{\Omega}_{n, j} \text { with } w \neq w^{\prime}
$$

To show the claim, we construct a finite subset of $\mathcal{Q}_{j}$, denoted by $W_{n}^{(j)}$, such that (i) $\# W_{n}^{(j)} \geq 7^{-j} M_{n}^{(j)}$, (ii) $\operatorname{diag}\left(\rho_{1}^{n}, \ldots, \rho_{j}^{n}\right) Q \cap K_{j} \neq \varnothing$ for each $Q \in$ $W_{n}^{(j)}$, and (iii) $2 Q \cap 2 \widetilde{Q}=\varnothing$ for $Q, \widetilde{Q} \in W_{n}^{(j)}$ with $Q \neq \widetilde{Q}$, where $2 Q:=$ $\bigcup_{Q^{\prime} \in \mathcal{Q}_{j}: Q^{\prime} \cap Q \neq \varnothing} Q^{\prime}$. For each $Q \in W_{n}^{(j)}$, since $\operatorname{diag}\left(\rho_{1}^{n}, \ldots, \rho_{j}^{n}\right) Q \cap K_{j} \neq \varnothing$, we can pick a word $w(Q) \in \Sigma_{n}$ such that $\operatorname{diag}\left(\rho_{1}^{n}, \ldots, \rho_{j}^{n}\right) Q \cap S_{w(Q)}^{(j)} K_{j} \neq \varnothing$ and hence

$$
\operatorname{diag}\left(\rho_{1}^{n}, \ldots, \rho_{j}^{n}\right) Q \cap S_{w(Q)}^{(j)}\left([0,1]^{j}\right) \neq \varnothing
$$

Denote $\bar{\Omega}_{n, j}=\left\{w(Q): Q \in W_{n}^{(j)}\right\}$. The separation condition (iii) for the elements in $W_{n}^{(j)}$ guarantees (9.3). This finishes the proof of the claim.

As above, we can construct $\bar{\Omega}_{n, j}$ well for each $j=1, \ldots, d$ and $n \in \mathbb{N}$. Now fix $n$ and consider the following collection of rectangles:

$$
\prod_{j=1}^{d} S_{w_{d} w_{d-1} \cdots w_{j}, j}([0,1]), \quad w_{j} \in \bar{\Omega}_{q_{j}(n), j}, 1 \leq j \leq d
$$


It is clear that the above rectangles are almost $\left(\rho_{d}\right)^{n}$-cubes and that each of them intersects with $K$. Furthermore, they are disjoint due to (9.3). Hence by the definition of box-counting dimension, we have

$$
\begin{aligned}
\underline{\operatorname{dim}}_{B}(K) \geq \liminf _{n \rightarrow \infty} \frac{\prod_{j=1}^{d} \# \bar{\Omega}_{q_{j}(n), j}}{-\log \left(\rho_{d}\right)^{n}} & \geq \liminf _{n \rightarrow \infty} \frac{\prod_{j=1}^{d} 7^{-j} M_{q_{j}(n)}^{(j)}}{-\log \left(\rho_{d}\right)^{n}} \\
& =\sum_{j=1}^{d}\left(\frac{1}{\lambda_{j}}-\frac{1}{\lambda_{j+1}}\right) H_{j} .
\end{aligned}
$$

This finishes the proof of (9.1).

Proof of THEOREM 2.15: We divide the proof into two steps:

Step 1. Show the variational principle for $\operatorname{dim}_{H} K$.

We first give an upper bound for $\operatorname{dim}_{H} K$. Fix $n \in \mathbb{N}$. Define

$$
N_{j}=\#\left\{S_{u}^{(j)}: u \in \Sigma_{n}\right\}, \quad j=1, \ldots, d,
$$

where $\left\{S_{i}^{(j)}\right\}_{i=1}^{\ell}$ denotes the IFS $\Phi_{1} \times \cdots \times \Phi_{j}$. Then we can construct

$$
\Omega_{j} \subset \Sigma_{n}, \quad j=d, d-1, \ldots, 1,
$$

such that $\# \Omega_{j}=N_{j}, \Sigma_{n} \supset \Omega_{d} \supset \Omega_{d-1} \supset \cdots \supset \Omega_{1}$ and furthermore, for each $u \in \Sigma_{n}$ and $1 \leq j \leq d$, there is $w_{j} \in \Omega_{j}$ such that $S_{u}^{(j)}=S_{w_{j}}^{(j)}$. Hence there are natural maps $\theta_{d}, \theta_{d-1}, \ldots, \theta_{1}$ with

$$
\Sigma_{n} \stackrel{\theta_{d}}{\longrightarrow} \Omega_{d} \stackrel{\theta_{d-1}}{\longrightarrow} \Omega_{d-1} \stackrel{\theta_{d-2}}{\longrightarrow} \cdots \stackrel{\theta_{1}}{\longrightarrow} \Omega_{1}
$$

such that $S_{u}^{(j)}=S_{\theta_{j}(u)}^{(j)}$ for any $1 \leq j \leq d$ and $u \in \Omega_{j+1}$, with the convention $\Omega_{d+1}=\Sigma_{n}$.

Let $Z_{d}: \Omega_{d} \rightarrow \mathbb{R}$ be the indicator of $\Omega_{d}$, i.e., $Z_{d}(u)=1$ for all $u \in \Omega_{d}$. Define

$$
Z_{d-1}(w)=\sum_{u \in \theta_{d-1}^{-1}(w)} Z_{d}(u), \quad w \in \Omega_{d-1} .
$$

Define inductively

$$
Z_{j}(w)=\sum_{u \in \theta_{j}^{-1}(w)} Z_{j+1}(u)^{\frac{\log \rho_{j+1}}{\log \rho_{j}+2}} \quad w \in \Omega_{j}, j=d-2, d-3, \ldots, 1 .
$$

In particular, define

$$
Z_{0}=\sum_{u \in \Omega_{1}} Z_{1}(u)^{\frac{\log \rho_{1}}{\log \rho_{2}}}
$$

Using the technique by Kenyon and Peres [33] (which is an extension of McMullen [44]), we have

$$
\operatorname{dim}_{H} K \leq \frac{\log Z_{0}}{-n \log \rho_{1}}
$$


More precisely, define a probability vector $(p(u))_{u \in \Omega_{d}}$ by

$$
p(u)=\frac{Z_{d}(u)}{Z_{d-1}\left(\theta_{d-1}(u)\right)} \cdot \prod_{j=1}^{d-1} \frac{Z_{j}\left(\theta_{j} \theta_{j+1} \cdots \theta_{d-1}(u)\right)^{\frac{\log \rho_{j}}{\log \rho_{j+1}}}}{Z_{j-1}\left(\theta_{j-1} \theta_{j} \cdots \theta_{d-1}(u)\right)}
$$

with convention $Z_{0}\left(\theta_{0} \cdots \theta_{d-1}(u)\right)=Z_{0}$ for any $u \in \Omega_{d}$. Let $v$ be the product measure on $\left(\Omega_{d}\right)^{\mathbb{N}}$ by assigning probability $p(u)$ to each digit $u \in \Omega_{d}$. The measure $v$ can be viewed as a measure on $\Sigma$, which is $\sigma^{n}$-invariant and ergodic. Let $\mu=v \circ \pi^{-1}$. Then

$$
\liminf _{r \rightarrow 0} \frac{\log \mu(B(\pi x, r))}{\log r} \leq \frac{\log Z_{0}}{-n \log \rho_{1}}, \quad x \in \Sigma .
$$

A detailed proof of (9.5) was given by Shmerkin (see the proof of (4.3) in [60]) for the case $d=2$, while a slight modification of the proof of [33, theorem 1.2] provides a proof of (9.5) for $d \geq 2$. Then (9.4) follows from (9.5) and Billingsley's lemma.

We want to indicate a connection between the upper bound $\log Z_{0} /\left(-n \log \rho_{1}\right)$ and the projection entropies. First, we define the projections $\theta_{j}^{*}: \Omega_{j+1}^{\mathbb{N}} \rightarrow \Omega_{j}^{\mathbb{N}}$, $j=d-1, \ldots, 1$ by

$$
\theta_{j}^{*}\left(\left(u_{k}\right)_{k=1}^{\infty}\right)=\left(\theta_{j}\left(u_{k}\right)\right)_{k=1}^{\infty}, \quad\left(u_{k}\right)_{k=1}^{\infty} \in \Omega_{j+1}^{\mathbb{N}} .
$$

Then it is easy to see that for each $1 \leq j \leq d-1$, the measure

$$
v_{j}:=v \circ\left(\theta_{j}^{*} \circ \theta_{j+1}^{*} \circ \cdots \circ \theta_{d-1}^{*}\right)^{-1}
$$

is a product measure on $\Omega_{j}^{\mathbb{N}}$.

Let $T_{j}$ denote the left shift operator on $\Omega_{j}^{\mathbb{N}}$. By a direct calculation, we have

$$
\frac{\log Z_{0}}{-n \log \rho_{1}}=\sum_{j=1}^{d}\left(\frac{1}{\lambda_{j}}-\frac{1}{\lambda_{j+1}}\right) \frac{h\left(T_{j}, v_{j}\right)}{n} .
$$

Thus we have

$$
\operatorname{dim}_{H} K \leq \sum_{j=1}^{d}\left(\frac{1}{\lambda_{j}}-\frac{1}{\lambda_{j+1}}\right) \frac{h\left(T_{j}, v_{j}\right)}{n} .
$$

Let $\tilde{\pi}_{j}, j=1, \ldots, d$, denote the canonical projection from $\Omega_{j}^{\mathbb{N}}$ to $\mathbb{R}^{j}$ with respect to the IFS $\left\{S_{u}^{(j)}\right\}_{u \in \Omega_{j}}$ (remember that $\pi_{j}$ denotes the canonical projection from $\Sigma$ to $\mathbb{R}^{d}$ with respect to $\left\{S_{u}^{(j)}: u \in \Sigma_{n}\right\}$ ). According to Lemma 4.23(ii), we have

$$
h_{\tilde{\pi}_{j}}\left(T_{j}, v_{j}\right)=h_{\pi_{j}}\left(\sigma^{n}, v\right), \quad j=1, \ldots, d .
$$


Since $\Phi_{1} \times \cdots \times \Phi_{j}, j=1, \ldots, d$, satisfy the AWSC, there is a sequence $\left(t_{n}\right)$ of positive integers with $\lim _{n} \log t_{n} / n=0$ such that

$$
\sup _{x \in \mathbb{R}^{j}} \#\left\{S_{u}^{(j)}: u \in \Omega_{j}, x \in S_{u}^{(j)}\left(K_{j}\right)\right\} \leq t_{n}, \quad j=1, \ldots, d,
$$

where $K_{j}$ denotes the attractor of $\Phi_{1} \times \cdots \times \Phi_{j}$. By Corollary 4.22, we have

$$
h_{\tilde{\pi}_{j}}\left(T_{j}, v_{j}\right) \geq h\left(T_{j}, v_{j}\right)-\log t_{n} \geq h\left(T_{j}, v_{j}\right)-\log t_{n} .
$$

This together with (9.7) yields $h_{\pi_{j}}\left(\sigma^{n}, v\right) \geq h\left(T_{j}, v_{j}\right)-\log t_{n}$. Now applying Theorem 2.11 to the IFS $\left\{S_{u}: u \in \Sigma_{n}\right\}$, we have

$$
\begin{aligned}
\operatorname{dim}_{H} v \circ \pi^{-1} & =\frac{1}{n} \sum_{j=1}^{d}\left(\frac{1}{\lambda_{j}}-\frac{1}{\lambda_{j+1}}\right) h_{\pi_{j}}\left(\sigma^{n}, v\right) \\
& \geq \frac{1}{n} \sum_{j=1}^{d}\left(\frac{1}{\lambda_{j}}-\frac{1}{\lambda_{j+1}}\right)\left(h\left(T_{j}, v_{j}\right)-\log t_{n}\right) \\
& \geq \operatorname{dim}_{H} K-\frac{\log t_{n}}{n} \cdot \sum_{j=1}^{d}\left(\frac{1}{\lambda_{j}}-\frac{1}{\lambda_{j+1}}\right) \quad \text { (by (9.6)) }
\end{aligned}
$$

Let $m=\frac{1}{n} \sum_{i=1}^{n} v \circ \sigma^{-i}$. Then $m$ is ergodic and $\operatorname{dim}_{H} m \circ \pi^{-1}=\operatorname{dim}_{H} v \circ \pi^{-1}$. Letting $n$ tend to $\infty$, we obtain

$$
\sup \left\{\operatorname{dim}_{H} m \circ \pi^{-1}: m \in \mathcal{M}_{\sigma}(\Sigma), m \text { is ergodic }\right\} \geq \operatorname{dim}_{H} K .
$$

It is clear the " $\geq$ " in the above inequality can be replaced by "=" since $m \circ \pi^{-1}$ is supported on $K$. Note that $h_{\pi_{j}}(\sigma, \cdot), j=1, \ldots, d$, are upper semicontinuous on $\mathcal{M}_{\sigma}(\Sigma)$ (see Proposition 4.20 and (9.8)). By Theorem 2.2(ii) and Theorem 2.11, we see that the supremum in (9.9) is attained at some ergodic element in $\mathcal{M}_{\sigma}(\Sigma)$. This finishes the proof of the variational principle for $\operatorname{dim}_{H} K$.

Step 2. Show the variational principle for $\operatorname{dim}_{B} K$.

By Lemma 9.2, we only need to show that under the assumption of Theorem 2.15,

$$
H_{j} \geq \tilde{H}_{j}, \quad j=1, \ldots, d,
$$

where

$$
H_{j}=\sup \left\{h_{\pi_{j}}(\sigma, m): m \in \mathcal{M}_{\sigma}(\Sigma)\right\}, \quad \tilde{H}_{j}=\lim _{n \rightarrow \infty} \frac{\log \#\left\{S_{u}^{(j)}: u \in \Sigma_{n}\right\}}{n} .
$$

To see (9.10), by (9.8) and Lemma 9.1, we have

$$
H_{j} \geq \frac{\log \#\left\{S_{u}^{(j)}: u \in \Sigma_{n}\right\}-\log t_{n}}{n}, \quad n \in \mathbb{N} .
$$

Letting $n \rightarrow \infty$, we obtain (9.10) by the assumption $\log t_{n} / n \rightarrow 0$. This finishes the proof of the theorem. 
Remark 9.3. With an essentially identical proof, Theorem 2.15 can be extended to the following class of IFSs $\Phi=\Phi_{1} \times \cdots \times \Phi_{k}$ on $\mathbb{R}^{q_{1}} \times \cdots \times \mathbb{R}^{q_{k}}$, where $\Phi_{j}$ has the form $\left\{A_{j} z_{j}+c_{i, j}\right\}_{i=1}^{\ell}$ such that $A_{j}$ is the inverse of an integral matrix and all the eigenvalues of $A_{j}$ equal $\rho_{j}$ in modulus, $\rho_{1}>\cdots>\rho_{k}, c_{i, j} \in \mathbb{Q}^{q_{j}}$.

This together with Lemma 7.2 and the proof of Theorem 2.12 yields the following:

THEOREM 9.4 Let $\Phi=\left\{S_{i}\right\}_{i=1}^{\ell}$ be an IFS on $\mathbb{R}^{d}$ of the form

$$
S_{i}(x)=A x+c_{i}, \quad i=1, \ldots, \ell,
$$

where $A$ is the inverse of an integral expanding $d \times d$ matrix, $c_{i} \in \mathbb{Z}^{d}$. Let $K$ be the attractor of the IFS. Then there is an ergodic measure on $K$ of full Hausdorff dimension.

\section{A Final Remark about Infinite Noncontractive IFSs}

In the previous sections, we have made the restriction that an IFS consists of finitely many contractive maps. We remark that part of our results can be extended to certain infinite noncontractive IFSs.

Let $\Phi=\left\{S_{i}\right\}_{i=1}^{\infty}$ be a family of maps on $\mathbb{R}^{d}$ of the form

$$
S_{i}(x)=\rho_{i} R_{i}(x)+a_{i}, \quad i=1,2, \ldots,
$$

where $\rho_{i}>0, R_{i}$ are orthogonal $d \times d$ matrices, and $a_{i} \in \mathbb{R}^{d}$.

Let $(X, \sigma)$ be the left shift over the alphabet $\{i: i \in \mathbb{N}\}$, and let $m$ be an ergodic measure on $X$ satisfying $H_{m}\left(\mathcal{P}_{\infty}\right)<\infty$, where $\mathcal{P}_{\infty}$ denotes the partition of $X$ given by

$$
\mathcal{P}_{\infty}=\{[i]: i \in \mathbb{N}\},
$$

where $[i]=\left\{\left(x_{i}\right)_{i=1}^{\infty} \in X: x_{1}=i\right\}$. Assume that $\Phi$ is $m$-contractive in the sense that

$$
\sum_{i=1}^{\infty}\left(\log \rho_{i}\right) m([i])<0, \quad \sum_{i=1}^{\infty}\left(\log \left|a_{i}\right|\right) m([i])<\infty
$$

Denote

$$
\lambda=-\sum_{i=1}^{\infty}\left(\log \rho_{i}\right) m([i]) .
$$

Let $X^{\prime}$ denote the set of points $x=\left(x_{i}\right)_{i=1}^{\infty} \in X$ such that

$$
\lim _{n \rightarrow \infty} \frac{1}{n} \log \left(\rho_{x_{1}} \rho_{x_{2}} \cdots \rho_{x_{n}}\right)=-\lambda, \quad \lim _{n \rightarrow \infty} \frac{1}{n} \log \left|a_{x_{n}}\right|=0 .
$$

Then $X^{\prime}$ satisfies $\sigma^{-1}\left(X^{\prime}\right)=X^{\prime}$. Furthermore, by Birkhoff's ergodic theorem,

$$
m\left(X^{\prime}\right)=1 .
$$


Define the projection map $\pi: X^{\prime} \rightarrow \mathbb{R}^{d}$ by

$$
\pi(x)=\lim _{n \rightarrow \infty} S_{x_{1}} \circ S_{x_{2}} \circ \cdots \circ S_{x_{n}}(0), \quad x \in X^{\prime} .
$$

It is easily checked that $\pi$ is well-defined. Let $\mu=m \circ \pi^{-1}$ be the projection of $m$ under $\pi$. We have the following theorem:

THEOREM 10.1 Under the above setting, $\mu=m \circ \pi^{-1}$ is exactly dimensional and

$$
\operatorname{dim}_{H} \mu=\frac{h_{\pi}(\sigma, m)}{\lambda},
$$

where $H_{\pi}(\sigma, m)=H_{m}\left(\mathcal{P}_{\infty} \mid \sigma^{-1} \pi^{-1} \gamma\right)-H_{m}\left(\mathcal{P}_{\infty} \mid \pi^{-1} \gamma\right), \gamma=\mathcal{B}\left(\mathbb{R}^{d}\right)$.

We remark that when $m$ is a Bernoulli product measure, $\mu=m \circ \pi^{-1}$ is the stationary measure of an affine random walk determined by $\Phi$ and $m$, and the decay property of $\mu$ at infinity has been extensively studied in the literature (cf. [23] and references therein).

The proof of Theorem 10.1 is essentially identical to that given in Section 6 . Indeed, we only need to replace $\Sigma$ in Section 6 by $X^{\prime}$, and replace "let $c>1$ so that $c \sup _{x \in \Sigma} \bar{\rho}(x)<1$ " in the proof of Theorem 6.2 by "let $1<c<e^{\lambda}$."

Acknowledgments. The authors are grateful to François Ledrappier for his encouragement and helpful comments. They are indebted to Eric Olivier for stimulating discussions of the variational principle about the Hausdorff dimension of self-affine sets, and to Wen Huang for the discussions of the entropy theory. They also thank Quansheng Liu, Emile Le Page, and Yong-Luo Cao for valuable comments, and Guo-Hua Zhang for critical reading of the manuscript. The first author was partially supported by RGC grants in the Hong Kong Special Administrative Region, China (projects CUHK400706, CUHK401008), the Fok Ying Tong Education Foundation, and NSFC Grant 10571100. The second author was partially supported by the National Science Foundation under grants DMS-0240097 and DMS-0503870.

\section{Bibliography}

[1] Barański, K. Hausdorff dimension of the limit sets of some planar geometric constructions. Adv. Math. 210 (2007), no. 1, 215-245.

[2] Barnsley, M. Fractals everywhere. Academic, Boston, 1988.

[3] Barral, J.; Mensi, M. Gibbs measures on self-affine Sierpiński carpets and their singularity spectrum. Ergodic Theory Dynam. Systems 27 (2007), no. 5, 1419-1443.

[4] Barreira, L.; Pesin, Y.; Schmeling, J. Dimension and product structure of hyperbolic measures. Ann. of Math. (2) 149 (1999), no. 3, 755-783.

[5] Bedford, T. Crinkly curves, Markov partitions and box dimension in self-similar sets. Doctoral dissertation, University of Warwick, United Kingdom, 1984.

[6] Bedford, T. Applications of dynamical systems theory to fractals-a study of cookie-cutter Cantor sets. Fractal geometry and analysis (Montreal, PQ, 1989), 1-44. NATO Advanced Science Institutes Series C: Mathematical and Physical Sciences, 346. Kluwer, Dordrecht, The Netherlands, 1991. 
[7] Billingsley, P. Probability and measure. 3rd ed. Wiley Series in Probability and Mathematical Statistics. Wiley, New York, 1995.

[8] Bogenschütz, T. Entropy, pressure, and a variational principle for random dynamical systems. Random Comput. Dynam. 1 (1992/93), no. 1, 99-116.

[9] Bowen, R. Equilibrium states and the ergodic theory of Anosov diffeomorphisms. Lecture Notes in Mathematics, 470. Springer, Berlin-New York, 1975.

[10] Eckmann, J.-P.; Ruelle, D. Ergodic theory of chaos and strange attractors. Rev. Modern Phys. 57 (1985), no. 3, part 1, 617-656.

[11] Falconer, K. J. The Hausdorff dimension of self-affine fractals. Math. Proc. Cambridge Philos. Soc. 103 (1988), no. 2, 339-350.

[12] Falconer, K. J. Dimensions and measures of quasi self-similar sets. Proc. Amer. Math. Soc. 106 (1989), no. 2, 543-554.

[13] Falconer, K. J. Fractal geometry, mathematical foundations and applications. Wiley, Chichester, 1990.

[14] Fan, A. H. Sur les dimensions de mesures. Studia Math. 111 (1994), no. 1, 1-17.

[15] Fan, A. H.; Lau, K. S.; Rao, H. Relationships between different dimensions of a measure. Monatsh. Math. 135 (2002), no. 3, 191-201.

[16] Feng, D.-J. The smoothness of $L^{q}$-spectrum of self-similar measures with overlaps. J. London Math. Soc. (2) 68 (2003), no. 1, 102-118.

[17] Feng, D.-J. The limited Rademacher functions and Bernoulli convolutions associated with Pisot numbers. Adv. Math. 195 (2005), no. 1, 24-101.

[18] Feng, D.-J. Gibbs properties of self-conformal measures and the multifractal formalism. Ergodic Theory Dynam. Systems 27 (2007), no. 3, 787-812.

[19] Feng, D.-J.; Wang, Y. A class of self-affine sets and self-affine measures. J. Fourier Anal. Appl. 11 (2005), no. 1, 107-124.

[20] Gatzouras, D.; Peres, Y. Invariant measures of full dimension for some expanding maps. Ergodic Theory Dynam. Systems 17 (1997), no. 1, 147-167.

[21] Gatzouras, D.; Peres, Y. The variational principle for Hausdorff dimension: a survey. Ergodic theory of $\mathbb{Z}^{d}$ actions (Warwick, 1993-1994), 113-125. London Mathematical Society Lecture Note Series, 228. Cambridge University Press, Cambridge, England, 1996.

[22] Geronimo, J. S.; Hardin, D. P. An exact formula for the measure dimensions associated with a class of piecewise linear maps. Fractal approximation. Constr. Approx. 5 (1989), no. 1, 89-98.

[23] Guivarc'h, Y.; Le Page, E. On spectral properties of a family of transfer operators and convergence to stable laws for affine random walks. Ergodic Theory Dynam. Systems 28 (2008), no. 2, 423-446.

[24] Hu, H. Dimensions of invariant sets of expanding maps. Comm. Math. Phys. 176 (1996), no. 2, 307-320.

[25] Huang, W.; Ye, X.; Zhang, G. A local variational principle for conditional entropy. Ergodic Theory Dynam. Systems 26 (2006), no. 1, 219-245.

[26] Hueter, I.; Lalley, S. P. Falconer's formula for the Hausdorff dimension of a self-affine set in $\mathbb{R}^{2}$. Ergodic Theory Dynam. Systems 15 (1995), no. 1, 77-97.

[27] Hutchinson, J. E. Fractals and self-similarity. Indiana Univ. Math. J. 30 (1981), no. 5, 713-747.

[28] Jordan, T.; Pollicott, M.; Simon, K. Hausdorff dimension for randomly perturbed self affine attractors. Comm. Math. Phys. 270 (2007), no. 2, 519-544.

[29] Käenmäki, A. On natural invariant measures on generalised iterated function systems. Ann. Acad. Sci. Fenn. Math. 29 (2004), no. 2, 419-458.

[30] Käenmäki, A.; Shmerkin, P. Overlapping self-affine sets of Kakeya type. Ergodic Theory Dynam. Systems, forthcoming. arXiv: 0710.0442v1, 2007.

[31] Keller, G. Equilibrium states in ergodic theory. London Mathematical Society Student Texts, 42. Cambridge University Press, Cambridge, England, 1998. 
[32] Kenyon, R.; Peres, Y. Hausdorff dimensions of sofic affine-invariant sets. Israel J. Math. 94 (1996), 157-178.

[33] Kenyon, R.; Peres, Y. Measures of full dimension on affine-invariant sets. Ergodic Theory Dynam. Systems 16 (1996), no. 2, 307-323.

[34] Lalley, S. P. $\beta$-expansions with deleted digits for Pisot numbers $\beta$. Trans. Amer. Math. Soc. 349 (1997), no. 11, 4355-4365.

[35] Lalley, S. P. Random series in powers of algebraic integers: Hausdorff dimension of the limit distribution. J. London Math. Soc. (2) 57 (1998), no. 3, 629-654.

[36] Lalley, S. P.; Gatzouras, D. Hausdorff and box dimensions of certain self-affine fractals. Indiana Univ. Math. J. 41 (1992), no. 2, 533-568.

[37] Lau, K. S.; Ngai, S. M. Multifractal measure and a weak separation condition. Adv. Math. 141 (1999), no. 1, 45-96.

[38] Ledrappier, F. On the dimension of some graphs. Symbolic dynamics and its applications (New Haven, CT, 1991), 285-293. Contemporary Mathematics, 135. American Mathematical Society, Providence, R.I., 1992.

[39] Ledrappier, F.; Porzio, A. A dimension formula for Bernoulli convolutions. J. Statist. Phys. 76 (1994), no. 5-6, 1307-1327.

[40] Ledrappier, F; Young, L.-S. The metric entropy of diffeomorphisms. I. Characterization of measures satisfying Pesin's entropy formula. II. Relations between entropy, exponents and dimension. Ann. of Math. (2) 122 (1985), no. 3, 509-539; 540-574.

[41] Luzia, N. A variational principle for the dimension for a class of non-conformal repellers. Ergodic Theory Dynam. Systems 26 (2006), no. 3, 821-845.

[42] Mañé, R. Ergodic theory and differentiable dynamics. Ergebnisse der Mathematik und ihrer Grenzgebiete (3), 8. Springer, Berlin, 1987.

[43] Mattila, P. Geometry of sets and measures in Euclidean spaces. Fractals and rectifiability. Cambridge Studies in Advanced Mathematics, 44. Cambridge University Press, Cambridge, England, 1995.

[44] McMullen, C. The Hausdorff dimension of general Sierpiński carpets. Nagoya Math. J. 96 (1984), 1-9.

[45] Ngai, S.-M.; Wang, Y. Hausdorff dimension of self-similar sets with overlaps. J. London Math. Soc. (2) 63 (2001), no. 3, 655-672.

[46] Olivier, E. Variational principle for dimension and the uniqueness of the measure with full dimension on $(\bmod 1)$ Sierpiński carpets. Preprint, 2008.

[47] Parry, W. Entropy and generators in ergodic theory. Benjamin, New York-Amsterdam, 1969.

[48] Parry, W. Topics in ergodic theory. Cambridge Tracts in Mathematics, 75. Cambridge University Press, Cambridge-New York, 1981.

[49] Patzschke, N. Self-conformal multifractal measures. Adv. in Appl. Math. 19 (1997), no. 4, 486513.

[50] Peres, Y.; Solomyak, B. Existence of $L^{q}$ dimensions and entropy dimension for self-conformal measures. Indiana Univ. Math. J. 49 (2000), no. 4, 1603-1621.

[51] Pesin, Y. B. Dimension theory in dynamical systems. Contemporary views and applications. Chicago Lectures in Mathematics. University of Chicago Press, Chicago, 1997.

[52] Przytycki, F.; Urbański, M. On the Hausdorff dimension of some fractal sets. Studia Math. 93 (1989), no. 2, 155-186.

[53] Qian, M.; Xie, J.-S. Entropy formula for endomorphisms: relations between entropy, exponents and dimension. Discrete Contin. Dyn. Syst. 21 (2008), no. 2, 367-392.

[54] Rao, H.; Wen, Z. Y. A class of self-similar fractals with overlap structure. Adv. in Appl. Math. 20 (1998), no. 1, 50-72.

[55] Rohlin, V. A. On the fundamental ideas of measure theory. Mat. Sbornik N.S. 25 (67) (1949), 107-150; translation in Amer. Math. Soc. Translation 1952, (1952), no. 71.

[56] Rudin, W. Real and complex analysis. 3rd ed. McGraw-Hill, New York, 1987. 
[57] Salem, R. Algebraic numbers and Fourier analysis. Heath, Boston, 1963.

[58] Schmeling, J. A dimension formula for endomorphisms - the Belykh family. Ergodic Theory Dynam. Systems 18 (1998), no. 5, 1283-1309.

[59] Schmeling, J.; Troubetzkoy, S. Dimension and invertibility of hyperbolic endomorphisms with singularities. Ergodic Theory Dynam. Systems 18 (1998), no. 5, 1257-1282.

[60] Shmerkin, P. Overlapping self-affine sets. Indiana Univ. Math. J. 55 (2006), no. 4, 1291-1331.

[61] Solomyak, B. Measure and dimension for some fractal families. Math. Proc. Cambridge Philos. Soc. 124 (1998), no. 3, 531-546.

[62] Stoll, R. R. Linear algebra and matrix theory. McGraw-Hill, New York-Toronto-London, 1952.

[63] Walters, P. An introduction to ergodic theory. Graduate Texts in Mathematics, 79. Springer, New York-Berlin, 1982.

[64] Yamamoto, T. On the extreme values of the roots of matrices. J. Math. Soc. Japan 19 (1967), 173-178.

[65] Young, L.-S. Dimension, entropy and Lyapunov exponents. Ergodic Theory Dynam. Systems 2 (1982), no. 1, 109-124.

DE-JUn FENG

The Chinese University of Hong Kong

Department of Mathematics

SHATIN

HONG KONG

E-mail: djfeng@

math. cuhk. edu.hk

Received August 2008.

Revised December 2008.

\section{HUYi Hu}

Michigan State University

Department of Mathematics

East Lansing, MI 48824

E-mail: hu@math.msu .edu 